\title{
Total Synthesis of Diospyrodin and Its Three Diastereomers
}

Takumi Fukuda, Masanori Nagatomo and Masayuki Inoue*

Graduate School of Pharmaceutical Sciences

The University of Tokyo, Hongo, Bunkyo-ku, Tokyo 113-0033, Japan

Fax: $(+81) 3-5841-0568$

E-mail: inoue@mol.f.u-tokyo.ac.jp

Supporting Information

49 pages

Contents:

page

1. Experimental procedures

S2

2. Comparison of ${ }^{1} \mathrm{H}$ and ${ }^{13} \mathrm{C}\left\{{ }^{1} \mathrm{H}\right\}$ NMR data of the reported and synthetic $\quad$ S20 diospyrodin nonaacetate (2) in $\mathrm{CDCl}_{3}$

3. Comparison of ${ }^{1} \mathrm{H}$ and ${ }^{13} \mathrm{C}\left\{{ }^{1} \mathrm{H}\right\}$ NMR data of the reported and synthetic $\quad$ S22 diospyrodin (1) in $\mathrm{D}_{2} \mathrm{O}$

$\begin{array}{lll}\text { 4. Structural determination of } \mathbf{3 - \alpha} & \text { S23 }\end{array}$

5. Structural determination of compounds $\mathbf{1 6}, \mathbf{1 7}, \mathbf{S 7}$, and S8 S25 by ${ }^{13} \mathrm{C}\left\{{ }^{1} \mathrm{H}\right\} \mathrm{NMR}$ analysis

$\begin{array}{ll}\text { 6. References } & \text { S26 }\end{array}$

$\begin{array}{ll}\text { 7. NMR charts } & \text { S27 }\end{array}$ 


\section{Experimental procedures}

General method: All reactions sensitive to air or moisture were carried out under argon atmosphere in dry solvents unless otherwise noted. $\mathrm{CH}_{2} \mathrm{Cl}_{2}$, THF, and toluene were purified by Glass Contour solvent dispensing system (Nikko Hansen \& Co., Ltd.). All other reagents were used as supplied. All reactions under heating conditions were performed in an oil bath. ED-OGR4 (EcoDesign, Inc., Japan) was used as the ozone generator. Analytical thin-layer chromatography (TLC) was performed using E. Merck Silica gel $60 \mathrm{~F}_{254}$ pre-coated plates $(0.25$ $\mathrm{mm}$ ). Preparative thin-layer chromatography (PTLC) was performed using Merck silica gel $60 \mathrm{~F}_{254}$ pre-coated plates $(0.5 \mathrm{~mm})$. Flash column chromatography was performed using 40 $50 \mu \mathrm{m}$ Silica Gel 60N (Kanto Chemical Co., Inc.) or $60 \mu \mathrm{m}$ amine-modified Silica Gel Chromatorex NH-DM2035 (Fuji Silysia Chemical Ltd.). Unless otherwise noted, Silica Gel $60 \mathrm{~N}$ was used for the purification. Optical rotations were measured on JASCO P-2200 polarimeter at ambient temperature using the sodium D line. Melting points were measured on Yanaco MP-J3 micro melting point apparatus and were uncorrected. Infrared (IR) spectra were recorded on JASCO FT/IR-4100 spectrometer as a thin film on $\mathrm{KBr}$ or $\mathrm{ZnSe} .{ }^{1} \mathrm{H}$ and ${ }^{13} \mathrm{C}\left\{{ }^{1} \mathrm{H}\right\}$ NMR spectra were recorded on JEOL JNM-ECX-500, or JNM-ECS-400 spectrometer. Chemical shifts were denoted in ppm on the $\delta$ scale relative to residual solvent peaks as internal standard: $\mathrm{CHCl}_{3}\left(\delta=7.26\right.$ for $\left.{ }^{1} \mathrm{H} \mathrm{NMR}\right), \mathrm{CDCl}_{3}\left(\delta=77.0\right.$ for $\left.{ }^{13} \mathrm{C}\left\{{ }^{1} \mathrm{H}\right\} \mathrm{NMR}\right), \mathrm{C}_{6} \mathrm{D}_{5} \mathrm{H}(\delta=7.16$ for ${ }^{1} \mathrm{H}$ NMR $), \mathrm{C}_{6} \mathrm{D}_{6}\left(\delta=128.0\right.$ for ${ }^{13} \mathrm{C}\left\{{ }^{1} \mathrm{H}\right\}$ NMR $), \mathrm{CD}_{2} \mathrm{HOD}\left(\delta=3.31\right.$ for ${ }^{1} \mathrm{H}$ NMR $), \mathrm{CD}_{3} \mathrm{OD}$ ( $\delta=49.0$ for ${ }^{13} \mathrm{C}\left\{{ }^{1} \mathrm{H}\right\}$ NMR), $\mathrm{D}_{2} \mathrm{O}$ (containing acetone as internal reference; $\delta=2.22$ for ${ }^{1} \mathrm{H}$ NMR and $\delta=215.94$ for $\left.{ }^{13} \mathrm{C}\left\{{ }^{1} \mathrm{H}\right\} \mathrm{NMR}\right)$. Signal patterns are indicated as s, singlet; $\mathrm{d}$, doublet; q, quartet; $\mathrm{m}$, multiplet. The carbon numbering of compounds corresponds to that of diospyrodin (1) unless otherwise noted (Figure S1). High-resolution mass spectra were measured on JEOL JMS-T100LP (ESI-TOF).

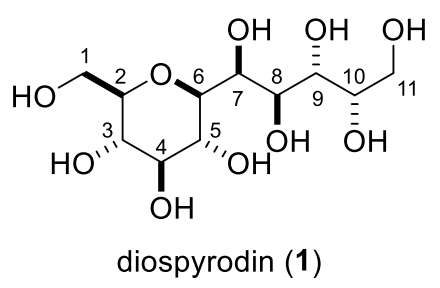

Figure S1. Numbering system of diospyrodin (1) 

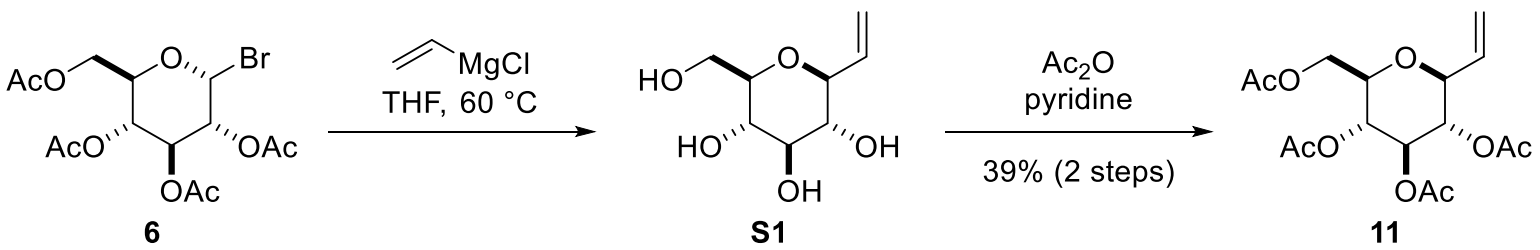

Alkene 11 [CAS: 51450-26-1]. ${ }^{\mathrm{S} 1} \quad$ Vinylmagnesium chloride (1.6 M in THF, $100 \mathrm{~mL}, 160$ mmol) was added to a solution of bromide $6(5.00 \mathrm{~g}, 12.2 \mathrm{mmol})$ in THF $(50 \mathrm{~mL})$ at $25{ }^{\circ} \mathrm{C}$. After the reaction mixture was stirred at $60{ }^{\circ} \mathrm{C}$ for $19 \mathrm{~h}, \mathrm{H}_{2} \mathrm{O}(25 \mathrm{~mL})$ and $5 \mathrm{M}$ aqueous $\mathrm{HCl}$ $(100 \mathrm{~mL})$ were successively added at $0{ }^{\circ} \mathrm{C}$. The resultant mixture was washed with $\mathrm{Et}_{2} \mathrm{O}(750$ $\mathrm{mL})$. The obtained aqueous layer was concentrated and azeotroped with toluene $(10 \mathrm{~mL} \times 3)$ to afford the crude tetraol S1 [CAS: 51450-27-2], ${ }^{\mathrm{S} 1}$ which was used in the next reaction without further purification.

$\mathrm{Ac}_{2} \mathrm{O}(150 \mathrm{~mL})$ was added to a solution of the above crude $\mathbf{S} 1$ in pyridine $(150 \mathrm{~mL})$ at $0{ }^{\circ} \mathrm{C}$. After being stirred for $12 \mathrm{~h}$, the reaction mixture was diluted with toluene $(100 \mathrm{~mL})$, concentrated, and azeotroped with toluene $(10 \mathrm{~mL} \times 3)$. The residue was purified by flash column chromatography on silica gel $(100 \mathrm{~g}$, hexane/EtOAc $=3 / 1)$ to afford alkene $11(1.71 \mathrm{~g}$, $4.77 \mathrm{mmol})$ in 39\% yield over 2 steps: colorless solid; ${ }^{1} \mathrm{H}$ NMR $\left(400 \mathrm{MHz}, \mathrm{CDCl}_{3}\right): \delta 2.00(3 \mathrm{H}$, s, $\left.\mathrm{CH}_{3} \mathrm{CO}\right), 2.01\left(3 \mathrm{H}, \mathrm{s}, \mathrm{CH}_{3} \mathrm{CO}\right), 2.03\left(3 \mathrm{H}, \mathrm{s}, \mathrm{CH}_{3} \mathrm{CO}\right), 2.09\left(3 \mathrm{H}, \mathrm{s}, \mathrm{CH}_{3} \mathrm{CO}\right), 3.70(1 \mathrm{H}, \mathrm{ddd}, J$ $=9.8,4.8,2.1 \mathrm{~Hz}, \mathrm{H} 2), 3.87(1 \mathrm{H}, \mathrm{dd}, J=9.6,7.3 \mathrm{~Hz}, \mathrm{H} 6), 4.12(1 \mathrm{H}, \mathrm{dd}, J=12.4,2.1 \mathrm{~Hz}, \mathrm{H} 1 \mathrm{a})$, $4.25(1 \mathrm{H}, \mathrm{dd}, J=12.4,4.8 \mathrm{~Hz}, \mathrm{H} 1 \mathrm{~b}), 4.94(1 \mathrm{H}, \mathrm{dd}, J=9.8,9.4 \mathrm{~Hz}, \mathrm{H} 5), 5.09$ (1H, dd, $J=9.8$, $9.4 \mathrm{~Hz}, \mathrm{H} 3), 5.23$ (1H, dd, $J=9.4,9.4 \mathrm{~Hz}, \mathrm{H} 4), 5.28$ (1H, d, $J=10.5 \mathrm{~Hz}, \mathrm{H} 8 \mathrm{a}), 5.36(1 \mathrm{H}, \mathrm{d}, J$ $=17.4 \mathrm{~Hz}, \mathrm{H} 8 \mathrm{~b}), 5.75(1 \mathrm{H}, \mathrm{ddd}, J=17.4,10.5,7.3 \mathrm{~Hz}, \mathrm{H} 7)$; HRMS (ESI-TOF) $[\mathrm{M}+\mathrm{Na}]^{+} \mathrm{m} / \mathrm{z}$ : Calcd for $\mathrm{C}_{16} \mathrm{H}_{22} \mathrm{O}_{9} \mathrm{Na}$ 381.1156; Found 381.1148. The analytical data of $\mathbf{1 1}$ were identical to those reported previously.

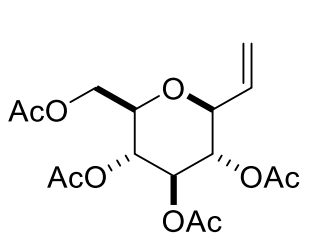

11

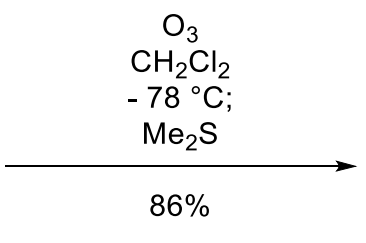<smiles>CC(=O)OCC1OC(C=O)C(OC(C)=O)C(OC(C)=O)C1OC(C)=O</smiles>

4

Aldehyde 4 [CAS: 83615-64-9]. ${ }^{\mathrm{S} 2} \quad \mathrm{O}_{3}$ was bubbled into a stirred solution of alkene 11 (1.31 $\mathrm{g}, 3.66 \mathrm{mmol})$ in $\mathrm{CH}_{2} \mathrm{Cl}_{2}(18.3 \mathrm{~mL})$ at $-78{ }^{\circ} \mathrm{C}$ for $5 \mathrm{~min}$. The excess $\mathrm{O}_{3}$ was removed by passing a stream of $\mathrm{Ar}$ at $-78{ }^{\circ} \mathrm{C}$ for $5 \mathrm{~min}$, and then $\mathrm{Me}_{2} \mathrm{~S}(6.10 \mathrm{~mL}, 82.5 \mathrm{mmol})$ was added to the mixture at $-78{ }^{\circ} \mathrm{C}$. The resultant solution was warmed to $25^{\circ} \mathrm{C}$ and stirred for $14 \mathrm{~h}$. The mixture was concentrated, and the residue was purified by flash column chromatography on 
silica gel (100 g, hexane/EtOAc $=2 / 1$ to $1 / 1)$ to afford aldehyde $4(1.28 \mathrm{~g}, 3.55 \mathrm{mmol})$ in $97 \%$ yield: colorless oil; ${ }^{1} \mathrm{H}$ NMR (400 MHz, $\left.\mathrm{C}_{6} \mathrm{D}_{6}\right): \delta 1.658\left(3 \mathrm{H}, \mathrm{s}, \mathrm{CH}_{3} \mathrm{CO}\right), 1.660\left(3 \mathrm{H}, \mathrm{s}, \mathrm{CH}_{3} \mathrm{CO}\right)$, $1.67\left(3 \mathrm{H}, \mathrm{s}, \mathrm{CH}_{3} \mathrm{CO}\right), 1.71\left(3 \mathrm{H}, \mathrm{s}, \mathrm{CH}_{3} \mathrm{CO}\right), 3.04$ (1H, ddd, $\left.J=10.0,5.0,2.3 \mathrm{~Hz}, \mathrm{H} 2\right), 3.19(1 \mathrm{H}$, dd, $J=10.1,2.3 \mathrm{~Hz}, \mathrm{H} 6), 3.95(1 \mathrm{H}, \mathrm{dd}, J=12.4,2.3 \mathrm{~Hz}, \mathrm{H} 1 \mathrm{a}), 4.21(1 \mathrm{H}, \mathrm{dd}, J=12.4,5.0 \mathrm{~Hz}$, H1b), 5.13-5.18 (2H, m, H3, H5), 5.29 (1H, dd, $J=9.6,9.6 \mathrm{~Hz}, \mathrm{H} 4), 9.18$ (1H, d, $J=2.3 \mathrm{~Hz}$, H7); HRMS (ESI-TOF) $[\mathrm{M}+\mathrm{Na}]^{+} \mathrm{m} / \mathrm{z}$ : Calcd for $\mathrm{C}_{15} \mathrm{H}_{20} \mathrm{O}_{10} \mathrm{Na}$ 383.0949; Found 383.0957. The analytical data of $\mathbf{4}$ were identical to those reported previously.

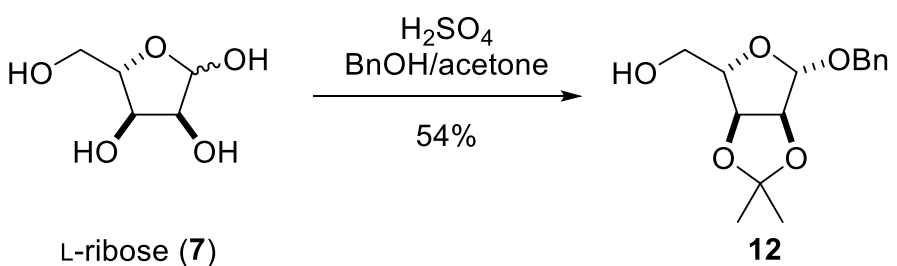

Alcohol 12. $\mathrm{H}_{2} \mathrm{SO}_{4}(18 \mathrm{M}, 300 \mu \mathrm{L})$ was added dropwise to a solution of L-ribose (7) (3.00 g, $20.0 \mathrm{mmol})$ in benzyl alcohol $(15.0 \mathrm{~mL})$ and acetone $(9.0 \mathrm{~mL})$ at $0{ }^{\circ} \mathrm{C}$. After the reaction mixture was stirred at $25^{\circ} \mathrm{C}$ for $19 \mathrm{~h}, \mathrm{Et}_{3} \mathrm{~N}(2.0 \mathrm{~mL})$ was added at $25^{\circ} \mathrm{C}$. The resultant mixture was concentrated. The residue was dissolved in EtOAc $(50 \mathrm{~mL})$, washed with saturated aqueous $\mathrm{NaHCO}_{3}(30 \mathrm{~mL})$ and brine $(30 \mathrm{~mL})$, dried over $\mathrm{Na}_{2} \mathrm{SO}_{4}$, filtered, and concentrated. The residue was purified by flash column chromatography on silica gel ( $200 \mathrm{~g}$, hexane/EtOAc $=8 / 1)$ to afford alcohol 12 (3.02 g, $10.8 \mathrm{mmol})$ in 54\% yield: colorless solid; m.p. 104-106 ${ }^{\circ} \mathrm{C}$; $[\alpha]_{\mathrm{D}}{ }^{27}+98.2\left(c 1.10, \mathrm{CHCl}_{3}\right)$; IR (film) 3486, 2929, 1374, 1210, 1159, 1101, 965, 866, $700 \mathrm{~cm}^{-}$ 1. ${ }^{1} \mathrm{H}$ NMR (400 MHz, $\mathrm{CDCl}_{3}$ ): $\delta 1.32$ (3H, s, $\mathrm{CH}_{3}$ of acetonide), 1.48 (3H, s, $\mathrm{CH}_{3}$ of acetonide), $3.14(1 \mathrm{H}, \mathrm{dd}, J=10.5,2.8 \mathrm{~Hz}, \mathrm{OH}), 3.64(1 \mathrm{H}, \mathrm{ddd}, J=10.5,10.5,2.8 \mathrm{~Hz}, \mathrm{H} 7 \mathrm{a}), 3.72(1 \mathrm{H}, \mathrm{ddd}$, $J=10.5,2.8,2.8 \mathrm{~Hz}, \mathrm{H} 7 \mathrm{~b}), 4.46(1 \mathrm{H}, \mathrm{dd}, J=2.8,2.8 \mathrm{~Hz}, \mathrm{H} 8), 4.58(1 \mathrm{H}, \mathrm{d}, J=11.9 \mathrm{~Hz}$, OCHaHbPh), 4.67 (1H, d, J=6.0 Hz, H9), $4.77(1 \mathrm{H}, \mathrm{d}, J=11.9 \mathrm{~Hz}, \mathrm{OCHaHbPh}), 4.86(1 \mathrm{H}, \mathrm{d}$, $J=6.0 \mathrm{~Hz}, \mathrm{H} 10), 5.18(1 \mathrm{H}, \mathrm{s}, \mathrm{H} 11), 7.32-7.39$ (5H, m, aromatic); ${ }^{13} \mathrm{C}\left\{{ }^{1} \mathrm{H}\right\} \mathrm{NMR}(100 \mathrm{MHz}$, $\left.\mathrm{CDCl}_{3}\right) \delta 24.6,26.3,64.0,70.1,81.5,85.9,88.4,108.0,112.1,128.18(2 \mathrm{C}), 128.23,128.6(2 \mathrm{C})$, 136.3; HRMS (ESI-TOF) [M+Na] $]^{+} \mathrm{m} / \mathrm{z}$ : Calcd for $\mathrm{C}_{15} \mathrm{H}_{20} \mathrm{O}_{5} \mathrm{Na} 303.1203$; Found 303.1200. 


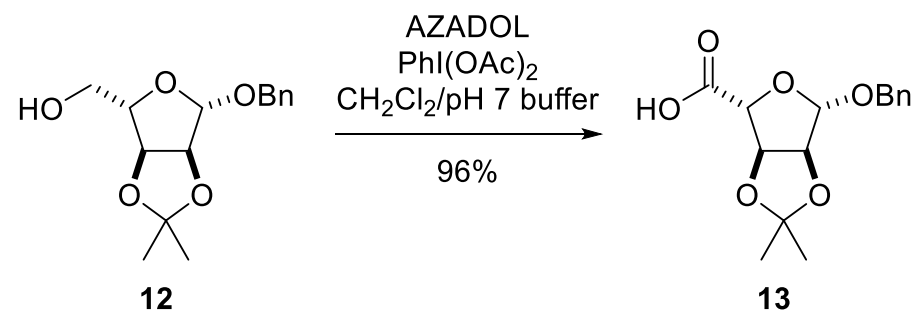

Carboxylic acid 13. 2-Hydroxy-2-azaadamantane (AZADOL, $48.3 \mathrm{mg}, 315 \mu \mathrm{mol}$ ) and $\mathrm{PhI}(\mathrm{OAc})_{2}(6.10 \mathrm{~g}, 18.9 \mathrm{mmol})$ were added to a solution of alcohol $12(1.77 \mathrm{~g}, 6.31 \mathrm{mmol})$ in $\mathrm{CH}_{2} \mathrm{Cl}_{2}(31.6 \mathrm{~mL})$ and $\mathrm{pH} 7$ phosphate buffer $(31.6 \mathrm{~mL})$ at $25{ }^{\circ} \mathrm{C}$. After the reaction mixture was stirred at $25{ }^{\circ} \mathrm{C}$ for $1.5 \mathrm{~h}$, saturated aqueous $\mathrm{Na}_{2} \mathrm{~S}_{2} \mathrm{O}_{3}(5 \mathrm{~mL})$ was added. The resultant solution was extracted with $\mathrm{CH}_{2} \mathrm{Cl}_{2}(30 \mathrm{~mL} \times 3)$. The combined organic layers were dried over $\mathrm{Na}_{2} \mathrm{SO}_{4}$, filtered, and concentrated. The residue was purified by flash chromatography on silica gel (30 g, hexane/EtOAc = 3/1 to EtOAc) to afford carboxylic acid $\mathbf{1 3}(1.78 \mathrm{~g}, 6.05 \mathrm{mmol})$ in 96\% yield: colorless solid; m.p. $94-95{ }^{\circ} \mathrm{C}$; $[\alpha]_{\mathrm{D}}{ }^{27}+103.5\left(c\right.$ 0.80, $\left.\mathrm{CHCl}_{3}\right)$; IR (film) 2940, 1723, 1379, 1211, 1090, 1052, 866, $699 \mathrm{~cm}^{-1} ;{ }^{1} \mathrm{H}$ NMR (400 MHz, $\left.\mathrm{CDCl}_{3}\right) \delta 1.33\left(3 \mathrm{H}, \mathrm{s}, \mathrm{CH}_{3}\right.$ of acetonide), $1.50\left(3 \mathrm{H}, \mathrm{s}, \mathrm{CH}_{3}\right.$ of acetonide), $4.53(1 \mathrm{H}, \mathrm{d}, J=11.9 \mathrm{~Hz}, \mathrm{OCHaHbPh}), 4.65(1 \mathrm{H}$, d, $J=6.0 \mathrm{~Hz}, \mathrm{H} 9$ or H10), $4.69(1 \mathrm{H}, \mathrm{s}, \mathrm{H} 8), 4.84(1 \mathrm{H}, \mathrm{d}, J=11.9 \mathrm{~Hz}, \mathrm{OCHa} H \mathrm{bPh}), 5.23(1 \mathrm{H}$, $\mathrm{d}, J=6.0 \mathrm{~Hz}, \mathrm{H} 10$ or H9), 5.25 (1H, s, H11), 7.27-7.37 (5H, m, aromatic); ${ }^{13} \mathrm{C}\left\{{ }^{1} \mathrm{H}\right\} \mathrm{NMR}(100$ $\left.\mathrm{MHz}, \mathrm{CDCl}_{3}\right) \delta 24.8,26.3,69.5,82.0,83.4,84.3,107.0,112.9,127.9,128.1(2 \mathrm{C}), 128.4(2 \mathrm{C})$, 136.7, 175.7; HRMS (ESI-TOF) [M-H] $]^{-} \mathrm{m} / \mathrm{z}$ : Calcd for $\mathrm{C}_{15} \mathrm{H}_{17} \mathrm{O}_{6} 293.1031$; Found 293.1023.
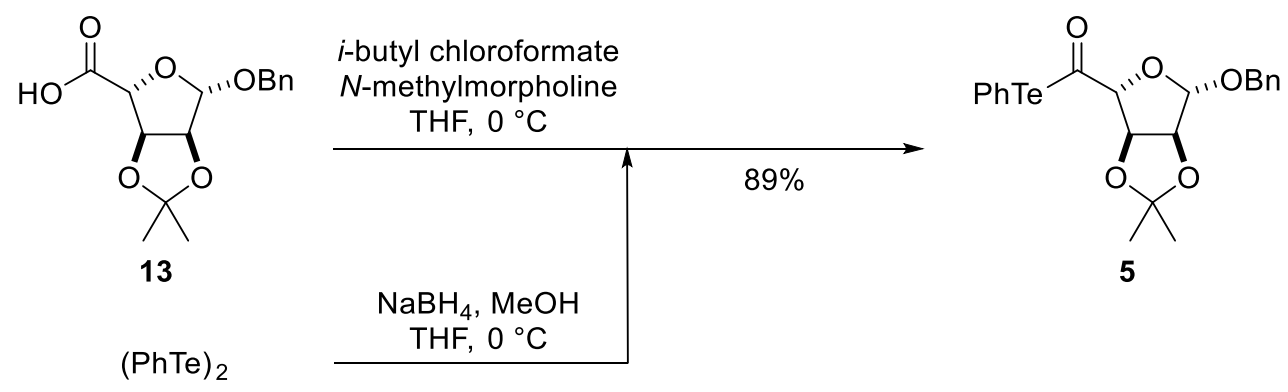

Acyl telluride 5. $N$-methylmorpholine $(717 \mu \mathrm{L}, 6.52 \mathrm{mmol})$ and isobutyl chloroformate (679 $\mu \mathrm{L}, 5.22 \mathrm{mmol})$ were added to a solution of carboxylic acid 13 (1.28 g, $4.35 \mathrm{mmol})$ in THF $(21.8 \mathrm{~mL})$ at $0{ }^{\circ} \mathrm{C} . \quad \mathrm{MeOH}(1.98 \mu \mathrm{L})$ was added to a suspension of $(\mathrm{PhTe})_{2}(1.78 \mathrm{~g}, 4.35 \mathrm{mmol})$ and $\mathrm{NaBH}_{4}(494 \mathrm{mg}, 13.1 \mathrm{mmol})$ in THF $(19.8 \mathrm{~mL})$ at $0{ }^{\circ} \mathrm{C}$. After both reaction mixtures were stirred at $0{ }^{\circ} \mathrm{C}$ for $30 \mathrm{~min}$, the latter reaction mixture was added dropwise to the former one at $0{ }^{\circ} \mathrm{C}$. The resultant mixture was stirred at $25^{\circ} \mathrm{C}$ for $30 \mathrm{~min}$, and then filtered through a pad of silica gel (15 g, EtOAc). The filtrate was concentrated, and the residue was purified 
by flash chromatography on silica gel ( $25 \mathrm{~g}$, hexane/EtOAc $=200 / 1$ to $10 / 1)$ to afford acyl telluride 5 (1.87 g, $3.88 \mathrm{mmol})$ in $89 \%$ yield: yellow oil; $[\alpha]_{\mathrm{D}}{ }^{25}+89.7\left(c 0.90, \mathrm{CHCl}_{3}\right)$; IR (film) 3060, 2986, 2935, 1706, 1377, 1213, 1084, 1036, 929, $731 \mathrm{~cm}^{-1}$; ${ }^{1} \mathrm{H}$ NMR (400 MHz, $\left.\mathrm{CDCl}_{3}\right) \delta 1.32\left(3 \mathrm{H}, \mathrm{s}, \mathrm{CH}_{3}\right.$ of acetonide), $1.49\left(3 \mathrm{H}, \mathrm{s}, \mathrm{CH}_{3}\right.$ of acetonide), $4.42(1 \mathrm{H}, \mathrm{d}, J=1.6$ Hz, H8), $4.63(1 \mathrm{H}, \mathrm{d}, J=5.9 \mathrm{~Hz}, \mathrm{H} 10), 4.65(1 \mathrm{H}, \mathrm{d}, J=11.7 \mathrm{~Hz}, \mathrm{OCHaHbPh}), 5.08(1 \mathrm{H}, \mathrm{dd}, J$ = 5.9, $1.6 \mathrm{~Hz}, \mathrm{H} 9), 5.13(1 \mathrm{H}, \mathrm{d}, J=11.7 \mathrm{~Hz}, \mathrm{OCHa} H \mathrm{bPh}), 5.35(1 \mathrm{H}, \mathrm{s}, \mathrm{H} 11), 7.28-7.40(8 \mathrm{H}$, $\mathrm{m}$, aromatic), 7.63-7.66 (2H, m, aromatic); ${ }^{13} \mathrm{C}\left\{{ }^{1} \mathrm{H}\right\} \mathrm{NMR}\left(100 \mathrm{MHz}, \mathrm{CDCl}_{3}\right) \delta 25.2,26.6,71.6$, 80.4, 84.6, 97.9, 108.7, 113.1, 113.6, 127.9 (2C), 128.0, 128.5 (2C), 128.8, 129.5 (2C), 136.8, 140.1 (2C), 206.1; HRMS (ESI-TOF) $[\mathrm{M}+\mathrm{Na}]^{+} \mathrm{m} / \mathrm{z}$ : Calcd for $\mathrm{C}_{21} \mathrm{H}_{22} \mathrm{O}_{5} \mathrm{TeNa}$ 507.0422; Found 507.0405 .
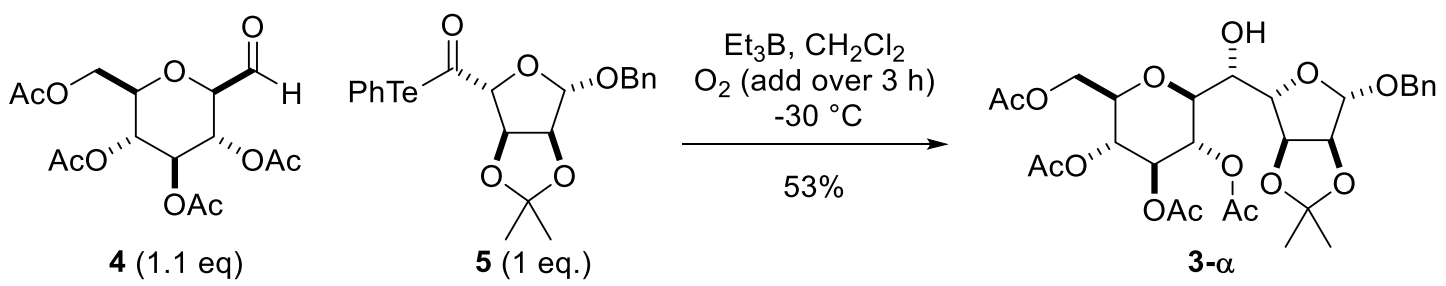

Alcohol 3- $\alpha$. $\quad \mathrm{Et}_{3} \mathrm{~B}(1.00 \mathrm{M}$ in hexane solution, $1.69 \mathrm{~mL}, 1.69 \mathrm{mmol})$ was added to a solution of acyl telluride 5 (404 mg, $838 \mu \mathrm{mol})$ and aldehyde 4 (332 mg, $921 \mu \mathrm{mol})$ in $\mathrm{CH}_{2} \mathrm{Cl}_{2}(4.19$ $\mathrm{mL})$ at $-30^{\circ} \mathrm{C} . \quad \mathrm{O}_{2}(9.39 \mathrm{~mL}, 419 \mu \mathrm{mol})$ was slowly added to the reaction mixture via syringe pump at $-30{ }^{\circ} \mathrm{C}$ over $3 \mathrm{~h}$. The reaction mixture was filtered through a pad of amine-modified silica gel (25 g, hexane to EtOAc). The filtrate was concentrated, and the residue was purified by flash column chromatography on silica gel $(50 \mathrm{~g}$, hexane/EtOAc $=3 / 1$ to $2 / 1)$ to afford alcohol 3- $\alpha$ (271 mg, $444 \mu \mathrm{mol})$ in 53\% yield: colorless solid; m.p. $152-154{ }^{\circ} \mathrm{C} ;[\alpha]_{\mathrm{D}}{ }^{24}+31.0$ (c 1.38, $\mathrm{CHCl}_{3}$ ); IR (film) 3476, 2942, 1756, 1372, 1231, 1035, 978, 868, $738 \mathrm{~cm}^{-1}$; ${ }^{1} \mathrm{H}$ NMR $\left(400 \mathrm{MHz}, \mathrm{CDCl}_{3}\right) \delta 1.32\left(3 \mathrm{H}, \mathrm{s}, \mathrm{CH}_{3}\right.$ of acetonide), $1.47\left(3 \mathrm{H}, \mathrm{s}, \mathrm{CH}_{3}\right.$ of acetonide), $1.71(3 \mathrm{H}$, s, $\left.\mathrm{CH}_{3} \mathrm{CO}\right), 2.00\left(3 \mathrm{H}, \mathrm{s}, \mathrm{CH}_{3} \mathrm{CO}\right), 2.03\left(3 \mathrm{H}, \mathrm{s}, \mathrm{CH}_{3} \mathrm{CO}\right), 2.08\left(3 \mathrm{H}, \mathrm{s}, \mathrm{CH} \mathrm{H}_{3} \mathrm{CO}\right), 3.45(1 \mathrm{H}, \mathrm{d}, J=$ $9.6 \mathrm{~Hz}, \mathrm{H} 7), 3.70(1 \mathrm{H}, \mathrm{d}, J=9.6 \mathrm{~Hz}, \mathrm{H} 6), 3.78$ (1H, ddd, $J=9.6,5.0,1.8 \mathrm{~Hz}, \mathrm{H} 2), 4.10(1 \mathrm{H}$, dd, $J=12.4,1.8 \mathrm{~Hz}, \mathrm{H1a}), 4.27-4.31$ (2H, m, H1b, H8), 4.51 (1H, d, $J=12.4 \mathrm{~Hz}, \mathrm{OCHaHbPh})$, $4.65(1 \mathrm{H}, \mathrm{d}, J=12.4 \mathrm{~Hz}, \mathrm{OCHa} H \mathrm{bPh}), 4.68(1 \mathrm{H}, \mathrm{d}, J=6.0 \mathrm{~Hz}, \mathrm{H} 10), 5.03(1 \mathrm{H}, \mathrm{d}, J=6.0 \mathrm{~Hz}$, H9), 5.07 (1H, dd, $J=9.6 \mathrm{~Hz}, \mathrm{H} 3), 5.16(1 \mathrm{H}, \mathrm{s}, \mathrm{H} 11), 5.19$ (1H, dd, $J=9.6 \mathrm{~Hz}, \mathrm{H} 5), 5.28(1 \mathrm{H}$, $\mathrm{dd}, J=9.6 \mathrm{~Hz}, \mathrm{H} 4), 7.29-7.37\left(5 \mathrm{H}, \mathrm{m}\right.$, aromatic); ${ }^{13} \mathrm{C}\left\{{ }^{1} \mathrm{H}\right\} \mathrm{NMR}\left(100 \mathrm{MHz}, \mathrm{CDCl}_{3}\right), \delta 20.2$, 20.59, 20.64, 20.7, 24.8, 26.3, 62.0, 68.1, 68.3, 68.6, 70.2, 74.2, 75.7, 76.0, 82.1, 85.2, 86.1, 108.3, 112.2, 127.6 (2C), 128.0, 128.6 (2C), 136.7, 169.4, 170.2, 170.4, 170.7; HRMS (ESI- 
TOF) $[\mathrm{M}+\mathrm{Na}]^{+} \mathrm{m} / \mathrm{z}$ : Calcd for $\mathrm{C}_{29} \mathrm{H}_{38} \mathrm{O}_{14} \mathrm{Na}$ 633.2154; Found 633.2149.
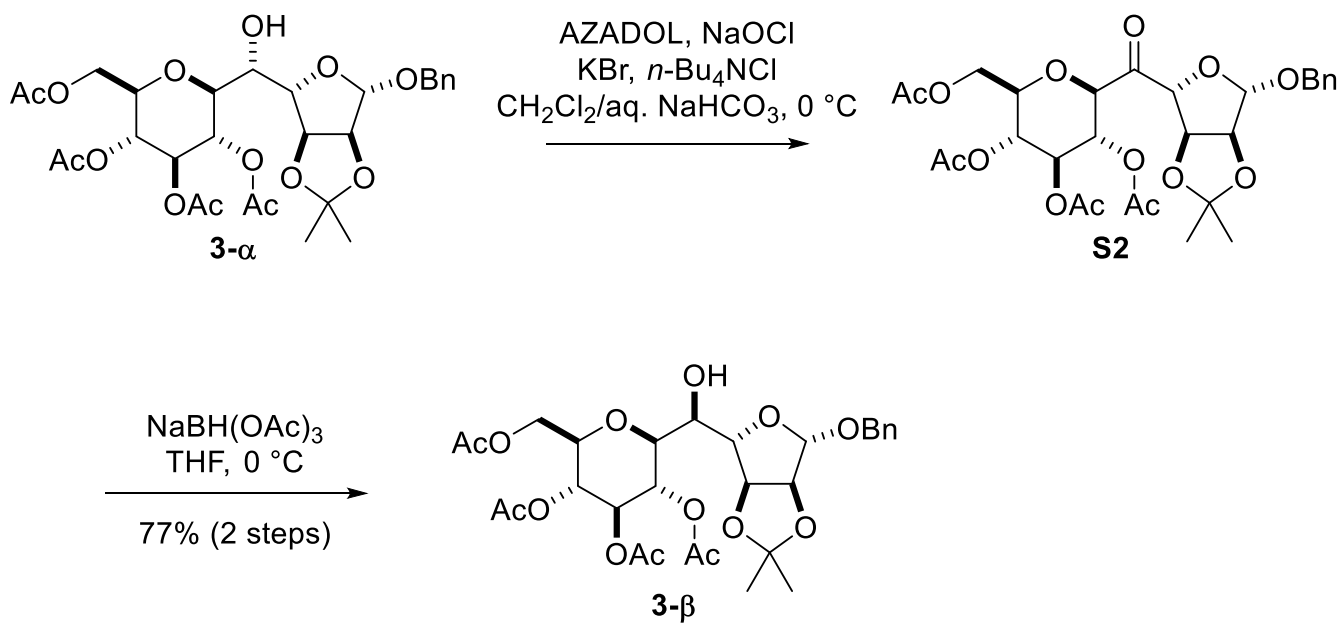

Alcohol 3- $\alpha$. Aqueous $\mathrm{NaOCl}(1.41 \mathrm{M}, 442 \mu \mathrm{L}, 623 \mu \mathrm{mol})$ was slowly added to a vigorously stirred solution of AZADOL (2.39 mg, $15.6 \mu \mathrm{mol}), \mathrm{KBr}(3.71 \mathrm{mg}, 31.1 \mu \mathrm{mol}), n$-Bu $4 \mathrm{NCl}(4.34$ $\mathrm{mg}, 15.6 \mu \mathrm{mol})$, and alcohol 3- $\alpha(95.3 \mathrm{mg}, 156 \mu \mathrm{mol})$ in saturated aqueous $\mathrm{NaHCO}_{3}(2.6 \mathrm{~mL})$ and $\mathrm{CH}_{2} \mathrm{Cl}_{2}(5.2 \mathrm{~mL})$ at $0{ }^{\circ} \mathrm{C}$. The mixture was stirred for $1.5 \mathrm{~h}$ at $0{ }^{\circ} \mathrm{C}$, and then saturated aqueous $\mathrm{Na}_{2} \mathrm{~S}_{2} \mathrm{O}_{3}(5 \mathrm{~mL})$ was added. The resultant mixture was extracted with $\mathrm{CH}_{2} \mathrm{Cl}_{2}$ (10 $\mathrm{mL} \times 3$ ). The combined organic layers were dried over $\mathrm{Na}_{2} \mathrm{SO}_{4}$, filtered, and concentrated to afford the crude ketone $\mathbf{S 2}(99.5 \mathrm{mg})$, which was used in the next reaction without further purification. HRMS (ESI-TOF) $[\mathrm{M}+\mathrm{Na}]^{+} \mathrm{m} / \mathrm{z}$ : Calcd for $\mathrm{C}_{29} \mathrm{H}_{36} \mathrm{O}_{14} \mathrm{Na}$ 631.1997; Found 631.1976.

$\mathrm{NaBH}(\mathrm{OAc})_{3}(331 \mathrm{mg}, 1.56 \mathrm{mmol})$ was added to a solution of the above crude $\mathbf{S 2}$ in THF (15.6 $\mathrm{mL}$ ) at $0{ }^{\circ} \mathrm{C}$. The reaction mixture was stirred at $0{ }^{\circ} \mathrm{C}$ for $3 \mathrm{~h}$, and then saturated aqueous $\mathrm{NH}_{4} \mathrm{Cl}(15 \mathrm{~mL})$ was added. The resultant mixture was extracted with EtOAc $(15 \mathrm{~mL} \times 3)$. The combined organic layers were washed with brine $(50 \mathrm{~mL})$, dried over $\mathrm{Na}_{2} \mathrm{SO}_{4}$, filtered, and concentrated. The residue was purified by flash column chromatography on silica gel ( $7.5 \mathrm{~g}$, hexane $/$ EtOAc $=3 / 1$ to $2 / 1)$ to afford alcohol $\mathbf{3}-\beta(73.5 \mathrm{mg}, 120 \mu \mathrm{mol})$ in $77 \%$ yield over 2 steps: colorless amorphous; $[\alpha]_{\mathrm{D}}{ }^{24}+16.5\left(c 0.88, \mathrm{CHCl}_{3}\right)$; IR (film) $3410,2943,1752,1433$, 1374, 1227, 1065, 907, $861 \mathrm{~cm}^{-1} ;{ }^{1} \mathrm{H}$ NMR (400 MHz, $\left.\mathrm{C}_{6} \mathrm{D}_{6}\right) \delta 1.08$ (3H, s, $\mathrm{CH}_{3}$ of acetonide), $1.41\left(3 \mathrm{H}, \mathrm{s}, \mathrm{CH}_{3}\right.$ of acetonide), $1.63\left(3 \mathrm{H}, \mathrm{s}, \mathrm{CH}_{3} \mathrm{CO}\right), 1.66\left(3 \mathrm{H}, \mathrm{s}, \mathrm{CH}_{3} \mathrm{CO}\right), 1.70(3 \mathrm{H}, \mathrm{s}$, $\left.\mathrm{CH}_{3} \mathrm{CO}\right), 1.71\left(3 \mathrm{H}, \mathrm{s}, \mathrm{CH}_{3} \mathrm{CO}\right), 3.21(1 \mathrm{H}, \mathrm{dd}, J=9.1 \mathrm{~Hz}, \mathrm{H} 6), 3.32(1 \mathrm{H}, \mathrm{ddd}, J=10.1,4.6,2.3$ Hz, H2), 3.47 (1H, ddd, $J=11.9,9.1,2.3 \mathrm{~Hz}, \mathrm{H} 7), 3.56(1 \mathrm{H}, \mathrm{d}, J=11.9 \mathrm{~Hz}, \mathrm{OH}), 3.86(1 \mathrm{H}, \mathrm{dd}$, $J=12.4,2.3 \mathrm{~Hz}, \mathrm{H1a}), 4.19$ (1H, d, $J=11.4 \mathrm{~Hz}, \mathrm{OCHaHbPh}), 4.26(1 \mathrm{H}, \mathrm{dd}, J=12.4,4.6 \mathrm{~Hz}$, 
H1b), $4.43(1 \mathrm{H}, \mathrm{d}, J=11.4 \mathrm{~Hz}, \mathrm{OCHa} H \mathrm{bPh}), 4.55(1 \mathrm{H}, \mathrm{d}, J=6.0 \mathrm{~Hz}, \mathrm{H} 10), 4.61(1 \mathrm{H}, \mathrm{d}, J=$ $6.0 \mathrm{~Hz}, \mathrm{H} 9), 4.89$ (1H, d, $J=2.3 \mathrm{~Hz}, \mathrm{H} 8), 5.20$ (1H, s, H11), $5.24(1 \mathrm{H}, \mathrm{dd}, J=10.1,10.1 \mathrm{~Hz}$, H3), 5.39 (1H, dd, $J=9.6,9.1 \mathrm{~Hz}, \mathrm{H} 5), 5.43(1 \mathrm{H}, \mathrm{dd}, J=10.1,9.6 \mathrm{~Hz}, \mathrm{H} 4), 7.08-7.24(5 \mathrm{H}, \mathrm{m}$, aromatic); ${ }^{13} \mathrm{C}\left\{{ }^{1} \mathrm{H}\right\} \mathrm{NMR}\left(100 \mathrm{MHz}, \mathrm{C}_{6} \mathrm{D}_{6}\right) \delta 20.15,20.26,20.28,20.6,24.5,26.5,61.9,68.9$, 71.4, 71.5, 73.3, 74.5, 76.1, 76.5, 82.8, 86.3, 88.3, 110.0, 112.1, 128.6, 128.7 (2C), 129.0 (2C), 136.7, 169.2, 169.4, 170.0 (2C); HRMS (ESI-TOF) $[\mathrm{M}+\mathrm{Na}]^{+} \mathrm{m} / \mathrm{z}$ : Calcd for $\mathrm{C}_{29} \mathrm{H}_{38} \mathrm{O}_{14} \mathrm{Na}$ 633.2154; Found 633.2125.

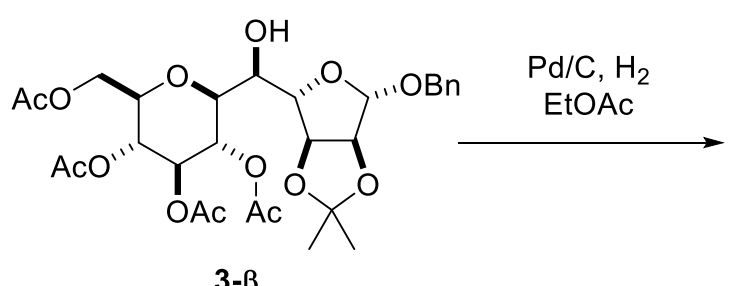<smiles>CC(=O)OCC1OC(C(O)C2O[C@@H](O)C3OC(C)(C)OC23)C(OC(C)=O)[C@H](OC(C)=O)C1OC(C)=O</smiles>

14

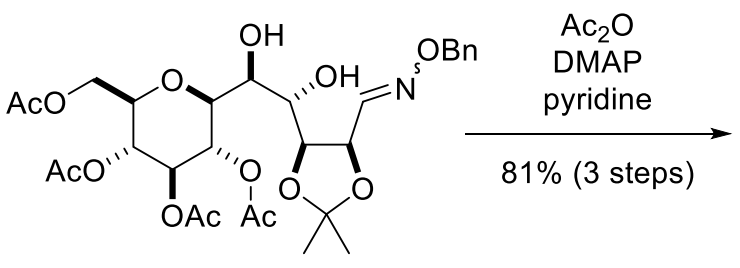

S3

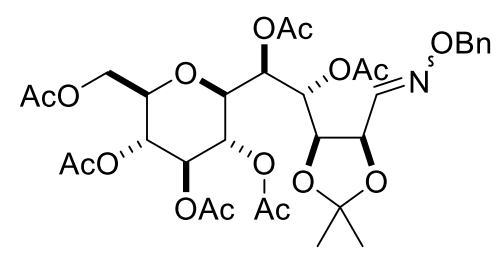

$15(E / Z=1: 0.2)$

Oxime 15. A suspension of benzyl acetal 3- $\beta(75.9 \mathrm{mg}, 124 \mu \mathrm{mol})$ and $\mathrm{Pd} / \mathrm{C}(10 \mathrm{wt} \% \mathrm{Pd}$ on carbon, $15.2 \mathrm{mg})$ in EtOAc $(2.48 \mathrm{~mL})$ was stirred under $\mathrm{H}_{2}$ atmosphere $(1 \mathrm{~atm})$ at $25^{\circ} \mathrm{C}$ for 12 h. The reaction mixture was then filtered through a pad of Celite $(2 \mathrm{~g})$ with EtOAc. The filtrate was concentrated to afford the crude hemiacetal $14(64.6 \mathrm{mg})$, which was used in the next reaction without further purification. HRMS (ESI-TOF) $[\mathrm{M}+\mathrm{Na}]^{+} \mathrm{m} / \mathrm{z}$ : Calcd for $\mathrm{C}_{22} \mathrm{H}_{32} \mathrm{O}_{14} \mathrm{Na}$ 543.1684; Found 543.1680.

$\mathrm{BnONH}_{2} \cdot \mathrm{HCl}(59.4 \mathrm{mg}, 372 \mu \mathrm{mol})$ was added to a solution of the above crude 14 in $\mathrm{CH}_{2} \mathrm{Cl}_{2}$ $(496 \mu \mathrm{L})$ and pyridine $(66.1 \mu \mathrm{L})$ at $25{ }^{\circ} \mathrm{C}$. After being stirred at $25{ }^{\circ} \mathrm{C}$ for $2 \mathrm{~d}$, the reaction mixture was concentrated. The residue was filtered through a pad of silica gel (1 g, EtOAc) to afford the crude oxime $\mathbf{S 3}(82.0 \mathrm{mg})$, which was used in the next reaction without further purification.

4-Dimethylaminopyridine (DMAP, $1.5 \mathrm{mg}, 12 \mu \mathrm{mol})$ and $\mathrm{Ac}_{2} \mathrm{O}(234 \mu \mathrm{L}, 2.48 \mathrm{mmol}$ ) were added to a solution of the above crude $\mathbf{S 3}$ in pyridine $(12.4 \mathrm{~mL})$ at $25^{\circ} \mathrm{C}$. After being stirred for $14 \mathrm{~h}$, the reaction mixture was diluted with toluene $(15 \mathrm{~mL})$ and concentrated. The residue was purified by flash column chromatography on silica gel ( $5 \mathrm{~g}$, hexane/EtOAc $=4 / 1$ to $1 / 1)$ to 
afford a $1: 0.2$ diastereomeric mixture of oxime $15(71.2 \mathrm{mg}, 100 \mu \mathrm{mol})$ in $81 \%$ yield over 3 steps: colorless oil; IR (film) 3638, 2987, 1752, 1434, 1372, 1222, 1038, 911, $738 \mathrm{~cm}^{-1} ;{ }^{1} \mathrm{H}$

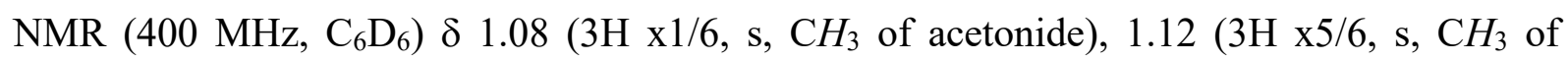
acetonide), 1.36 ( $3 \mathrm{H} \mathrm{x} 5 / 6, \mathrm{~s}, \mathrm{CH}_{3}$ of acetonide), 1.41 ( $3 \mathrm{H} \mathrm{x1/6,} \mathrm{s,} \mathrm{CH}_{3}$ of acetonide), 1.64-1.81 $\left(18 \mathrm{H}, \mathrm{m}, \mathrm{CH}_{3} \mathrm{CO} \times 6\right), 3.07-3.11(1 \mathrm{H}, \mathrm{m}, \mathrm{H} 2), 3.83-3.97$ (2H, m, H1a, H6), 4.36-4.42 (1H x1/6 and $2 \mathrm{H} \mathrm{x} 5 / 6, \mathrm{~m}, \mathrm{H} 1 \mathrm{~b}$ and $\mathrm{H} 1 \mathrm{~b}, \mathrm{H} 9), 4.56$ (1H x1/6, dd, $J=7.6,4.4 \mathrm{~Hz}, \mathrm{H} 9), 4.84(1 \mathrm{H} \mathrm{x} 5 / 6$, dd, $J=8.7,5.9 \mathrm{~Hz}, \mathrm{H} 10), 5.01\left(2 \mathrm{H} \mathrm{x} 5 / 6, \mathrm{~s}, \mathrm{OCH}_{2} \mathrm{Ph}\right), 5.03\left(2 \mathrm{H} \mathrm{x1} / 6, \mathrm{~s}, \mathrm{OCH}_{2} \mathrm{Ph}\right), 5.25-5.30(1 \mathrm{H}$, m, H3), 5.40-5.50 (2H x1/6 and $1 \mathrm{H} \mathrm{x5/6,} \mathrm{m,} \mathrm{H4,} \mathrm{H10} \mathrm{and} \mathrm{H4),} 5.61(1 \mathrm{H} \mathrm{x} 5 / 6$, dd, $J=3.2,2.3$ $\mathrm{Hz}, \mathrm{H} 7), 5.76-5.88$ (2H, m, H5, H8), 7.03-7.13 (4H x1/6 and 3H x5/6, m, H11, aromatic and aromatic), 7.25-7.29 (2H, m, aromatic), $7.75(1 \mathrm{H} \mathrm{x5/6,} \mathrm{d,} J=8.7 \mathrm{~Hz}, \mathrm{H} 11)$; Detectable signals of ${ }^{13} \mathrm{C}\left\{{ }^{1} \mathrm{H}\right\}$ NMR $\left(100 \mathrm{MHz}, \mathrm{C}_{6} \mathrm{D}_{6}\right), \delta 20.14,20.16,20.21,20.28,20.35,20.37,20.48,20.52$, 20.8, 21.1, 25.12, 25.15, 27.3, 27.5, 61.6, 61.7, 67.7, 67.9, 68.0, 69.7, 70.1, 70.6, 70.7, 71.1, 74.7, 74.8, 74.9, 75.5, 75.8, 76.4, 76.7, 77.0, 77.6, 77.8, 109.9, 110.4, 127.88, 127.94, 128.07, 128.12, 128.4, 128.53, 128.57, 128.64, 137.9, 147.0, 147.6, 169.0, 169.25, 169.27, 169.8, 170.0, 170.1, 170.2; HRMS (ESI-TOF) $[\mathrm{M}+\mathrm{Na}]^{+} \mathrm{m} / \mathrm{z}$ : Calcd for $\mathrm{C}_{33} \mathrm{H}_{43} \mathrm{NO}_{16} \mathrm{Na}$ 732.2474; Found 732.2453.
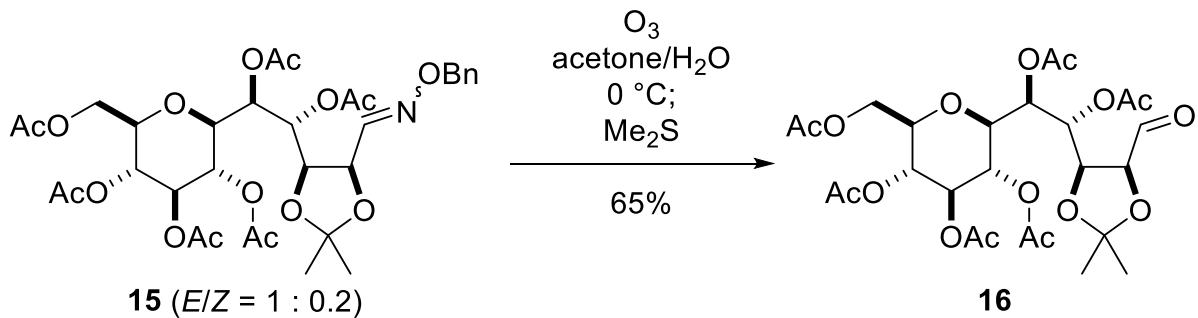

16

Aldehyde 16. $\mathrm{O}_{3}$ was bubbled into a solution of oxime $15(27.0 \mathrm{mg}, 38.0 \mu \mathrm{mol})$ in acetone $(11.4 \mathrm{~mL})$ and $\mathrm{H}_{2} \mathrm{O}(1.26 \mathrm{~mL})$ for $1 \mathrm{~h}$ at $0{ }^{\circ} \mathrm{C}$. The excess $\mathrm{O}_{3}$ was removed by passing a stream of $\mathrm{Ar}$ at $25{ }^{\circ} \mathrm{C}$ for $10 \mathrm{~min}$, and then $\mathrm{Me}_{2} \mathrm{~S}(1.26 \mathrm{~mL}, 17.0 \mathrm{mmol})$ was added to the mixture. The reaction mixture was warmed to $25^{\circ} \mathrm{C}$ and stirred for $13 \mathrm{~h}$. The mixture was concentrated, and the residue was purified by flash column chromatography on silica gel (2.5 g, hexane/EtOAc $=2 / 1$ to $1 / 1)$ to afford aldehyde $16(14.9 \mathrm{mg}, 24.6 \mu \mathrm{mol})$ in $65 \%$ yield: colorless oil; $[\alpha]_{\mathrm{D}}^{25}+2.9\left(c 0.75, \mathrm{CHCl}_{3}\right)$; IR (film) 3486, 2927, 1751, 1434, 1374, 1223, 1038, $907 \mathrm{~cm}^{-}$ 1; ${ }^{1} \mathrm{H}$ NMR (400 MHz, $\left.\mathrm{C}_{6} \mathrm{D}_{6}\right) \delta 1.09$ (3H, s, $\mathrm{CH}_{3}$ of acetonide), 1.43 (3H, s, $\mathrm{CH}_{3}$ of acetonide), $1.66\left(6 \mathrm{H}, \mathrm{s}, \mathrm{CH}_{3} \mathrm{CO} \times 2\right), 1.76\left(6 \mathrm{H}, \mathrm{s}, \mathrm{CH}_{3} \mathrm{CO} \times 2\right), 1.77\left(3 \mathrm{H}, \mathrm{s}, \mathrm{CH}_{3} \mathrm{CO}\right), 1.82\left(3 \mathrm{H}, \mathrm{s}, \mathrm{CH}_{3} \mathrm{CO}\right)$, $3.07(1 \mathrm{H}, \mathrm{m}, \mathrm{H} 2), 3.76(1 \mathrm{H}, \mathrm{dd}, J=9.6,3.7 \mathrm{~Hz}, \mathrm{H} 6), 3.84$ (1H, dd, $J=12.8,1.8 \mathrm{~Hz}, \mathrm{H} 1 \mathrm{a}), 4.19$ $(1 \mathrm{H}, \mathrm{dd}, J=6.9,3.6 \mathrm{~Hz}, \mathrm{H} 10), 4.34(1 \mathrm{H}, \mathrm{dd}, J=12.8,4.6 \mathrm{~Hz}, \mathrm{H} 1 \mathrm{~b}) 4.39$ (1H, dd, $J=7.8,6.9$ 
Hz, H9), 5.23 (1H, dd, $J=9.6,9.6 \mathrm{~Hz}, \mathrm{H} 3), 5.39$ (1H, dd, $J=9.6,9.6 \mathrm{~Hz}, \mathrm{H} 4), 5.51(1 \mathrm{H}, \mathrm{dd}, J$ = 3.7, $2.3 \mathrm{~Hz}, \mathrm{H} 7), 5.62(1 \mathrm{H}, \mathrm{dd}, J=9.6,9.6 \mathrm{~Hz}, \mathrm{H} 5), 5.94(1 \mathrm{H}, \mathrm{dd}, J=7.8,2.3 \mathrm{~Hz}, \mathrm{H} 8), 9.67$ $(1 \mathrm{H}, \mathrm{d}, J=3.6 \mathrm{~Hz}, \mathrm{H} 11) ;{ }^{13} \mathrm{C}\left\{{ }^{1} \mathrm{H}\right\} \mathrm{NMR}\left(100 \mathrm{MHz}, \mathrm{C}_{6} \mathrm{D}_{6}\right), \delta 20.36,20.42,20.57,20.61,20.68$, 20.9, 25.3, 27.3, 61.7, 68.0, 68.2, 70.2, 70.9, 75.0, 76.9, 77.4, 77.7, 81.4, 111.5, 169.2, 169.5, 169.6 (2C), 170.2, 170.3, 198.1; HRMS (ESI-TOF) $[\mathrm{M}+\mathrm{Na}]^{+} \mathrm{m} / \mathrm{z}$ : Calcd for $\mathrm{C}_{26} \mathrm{H}_{36} \mathrm{O}_{16} \mathrm{Na}$ 627.1896; Found 627.1871.
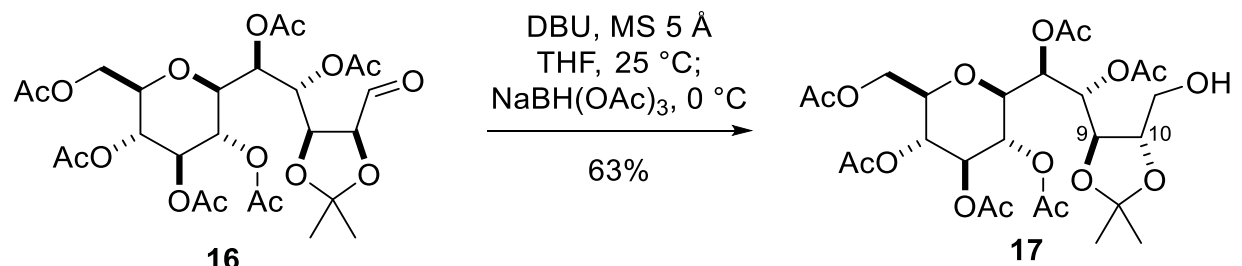

Alcohol 17. Molecular sieves $5 \AA$ A (MS $5 \AA$ A, $143 \mathrm{mg}$ ) was added to a solution of aldehyde 16 (14.3 mg, $23.7 \mu \mathrm{mol})$ in THF $(2.37 \mathrm{~mL})$ at $25{ }^{\circ} \mathrm{C}$. After the resultant mixture was stirred at $25^{\circ} \mathrm{C}$ for $2 \mathrm{~h}, 1,8$-diazabicyclo[5.4.0]undec-7-ene (DBU, $7.1 \mu \mathrm{L}, 48 \mu \mathrm{mol}$ ) was added. The reaction mixture was stirred at $25^{\circ} \mathrm{C}$ for $16 \mathrm{~h}$, and then $\mathrm{NaBH}(\mathrm{OAc})_{3}(100 \mathrm{mg}, 472 \mu \mathrm{mol})$ was added at $0{ }^{\circ} \mathrm{C}$. After the reaction mixture was stirred at $0{ }^{\circ} \mathrm{C}$ for $1 \mathrm{~h}$, saturated aqueous $\mathrm{NH}_{4} \mathrm{Cl}$ (3 mL) was added. The resultant mixture was extracted with EtOAc (3 mL x3). The combined organic layers were washed with brine $(10 \mathrm{~mL})$, dried over $\mathrm{Na}_{2} \mathrm{SO}_{4}$, filtered, and concentrated. The residue was purified by flash column chromatography on silica gel ( $2 \mathrm{~g}$, hexane $/$ EtOAc $=1 / 1$ to $1 / 2)$ to afford alocohol $17(9.11 \mathrm{mg}, 15.0 \mu \mathrm{mol})$ in $63 \%$ yield: colorless oil; $[\alpha]_{\mathrm{D}}{ }^{25}-13.1\left(c 0.75, \mathrm{CHCl}_{3}\right)$; IR (film) 3497, 2942, 1750, 1434, 1374, 1224, 1042, $906 \mathrm{~cm}^{-}$ ${ }^{1} ;{ }^{1} \mathrm{H}$ NMR (500 MHz, $\left.\mathrm{C}_{6} \mathrm{D}_{6}\right) \delta 1.33$ (3H, s, $\mathrm{CH}_{3}$ of acetonide), 1.41 (3H, s, $\mathrm{CH}_{3}$ of acetonide), $1.66\left(3 \mathrm{H}, \mathrm{s}, \mathrm{CH} \mathrm{C}_{3} \mathrm{CO}\right), 1.67\left(3 \mathrm{H}, \mathrm{s}, \mathrm{CH}_{3} \mathrm{CO}\right), 1.69\left(3 \mathrm{H}, \mathrm{s}, \mathrm{CH}_{3} \mathrm{CO}\right), 1.73\left(3 \mathrm{H}, \mathrm{s}, \mathrm{CH}_{3} \mathrm{CO}\right), 1.76$ $\left(3 \mathrm{H}, \mathrm{s}, \mathrm{CH}_{3} \mathrm{CO}\right), 1.81\left(3 \mathrm{H}, \mathrm{s}, \mathrm{CH}_{3} \mathrm{CO}\right), 3.06(1 \mathrm{H}, \mathrm{ddd}, J=9.8,4.6,2.0 \mathrm{~Hz}, \mathrm{H} 2), 3.49(1 \mathrm{H}, \mathrm{m}$, H11a), 3.65 (1H, m, H11b), 3.85 (1H, dd, $J=10.3,3.4 \mathrm{~Hz}, \mathrm{H} 6), 3.87$ (1H, dd, $J=12.3,2.0 \mathrm{~Hz}$, H1a), 4.06 (1H, dd, $J=8.0,3.9 \mathrm{~Hz}, \mathrm{H} 10), 4.29$ (1H, dd, $J=8.0,8.0 \mathrm{~Hz}, \mathrm{H} 9), 4.32$ (1H, dd, $J=$ 12.3, 4.6 Hz, H1b), 5.25 (1H, dd, $J=9.8,9.5 \mathrm{~Hz}, \mathrm{H} 3), 5.40$ (1H, dd, $J=9.5,9.2 \mathrm{~Hz}, \mathrm{H} 4), 5.64$ $(1 \mathrm{H}, \mathrm{dd}, J=3.4,2.9 \mathrm{~Hz}, \mathrm{H} 7), 5.67(1 \mathrm{H}, \mathrm{dd}, J=10.3,9.2 \mathrm{~Hz}, \mathrm{H} 5), 5.82(1 \mathrm{H}, \mathrm{dd}, J=8.0,2.9 \mathrm{~Hz}$, $\mathrm{H} 8) ;{ }^{13} \mathrm{C}\left\{{ }^{1} \mathrm{H}\right\} \mathrm{NMR}\left(125 \mathrm{MHz}, \mathrm{C}_{6} \mathrm{D}_{6}\right), \delta 20.1,20.2,20.31$ (2C), 20.34, 20.5, 26.7, 27.1, 61.6, $62.4,68.0,69.6,70.4,70.9,74.4,74.8,76.7,78.1,80.3,110.0,169.0,169.2,169.4,169.5$, 170.01, 170.06; HRMS (ESI-TOF) $[\mathrm{M}+\mathrm{Na}]^{+} \mathrm{m} / \mathrm{z}$ : Calcd for $\mathrm{C}_{26} \mathrm{H}_{38} \mathrm{O}_{16} \mathrm{Na}$ 629.2052; Found 629.2049 . 


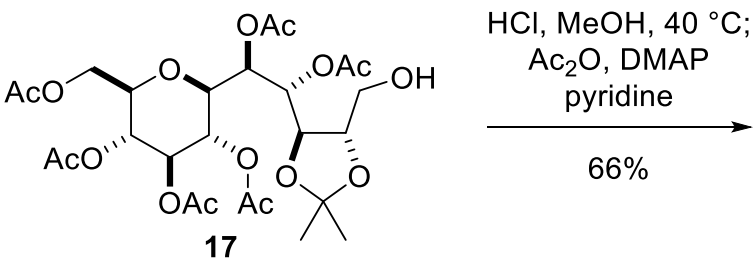

Diospyrodin nonaacetate (2) [CAS: 906088-85-5]. ${ }^{\mathrm{S} 3}$

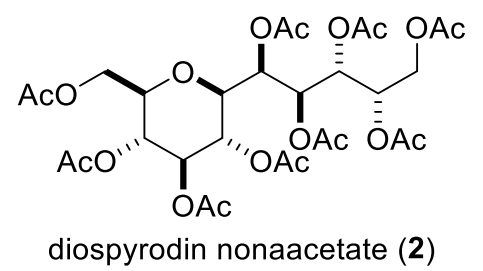

A solution of alcohol $17(16.3 \mathrm{mg}$, $26.9 \mu \mathrm{mol})$ in $0.5 \mathrm{M}$ solution of $\mathrm{HCl}$ in $\mathrm{MeOH}(2.68 \mathrm{~mL})$ was stirred at $40{ }^{\circ} \mathrm{C}$ for $2 \mathrm{~h}$. The reaction mixture was concentrated, and the residue was dissolved in pyridine. DMAP (3.3 mg, $27 \mu \mathrm{mol})$ and $\mathrm{Ac}_{2} \mathrm{O}(127 \mu \mathrm{L}, 1.34 \mathrm{mmol})$ were successively added to the resultant solution at $0{ }^{\circ} \mathrm{C}$. After the reaction mixture was stirred at $25^{\circ} \mathrm{C}$ for $16 \mathrm{~h}, \mathrm{MeOH}(1 \mathrm{~mL})$ was added. The resultant solution was concentrated and azeotroped with toluene $(1 \mathrm{~mL})$. The residue was purified by PTLC (hexane/EtOAc $=1 / 1)$ to afford diospyrodin nonaacetate $2(12.3 \mathrm{mg}, 17.8$ $\mu \mathrm{mol})$ in $66 \%$ yield: colorless amorphous; $[\alpha]_{\mathrm{D}}{ }^{18}-23.6\left(c 0.62, \mathrm{CHCl}_{3}\right)$; IR (film) 2963,1749 , 1433, 1372, 1219, 1038, 907, $736 \mathrm{~cm}^{-1} ;{ }^{1} \mathrm{H}$ NMR (500 MHz, $\left.\mathrm{CDCl}_{3}\right) \delta 1.99\left(3 \mathrm{H}, \mathrm{s}, \mathrm{CH}_{3} \mathrm{CO}\right)$, $2.00\left(3 \mathrm{H}, \mathrm{s}, \mathrm{CH} \mathrm{H}_{3} \mathrm{CO}\right), 2.01\left(3 \mathrm{H}, \mathrm{s}, \mathrm{CH} \mathrm{H}_{3} \mathrm{CO}\right), 2.06\left(6 \mathrm{H}, \mathrm{s}, \mathrm{CH}_{3} \mathrm{CO} \times 2\right), 2.07\left(3 \mathrm{H}, \mathrm{s}, \mathrm{CH}_{3} \mathrm{CO}\right)$, $2.08\left(3 \mathrm{H}, \mathrm{s}, \mathrm{CH} \mathrm{CH}_{3} \mathrm{CO}, 2.11\left(3 \mathrm{H}, \mathrm{s}, \mathrm{CH}_{3} \mathrm{CO}\right), 2.14\left(3 \mathrm{H}, \mathrm{s}, \mathrm{CH}_{3} \mathrm{CO}\right), 3.49(1 \mathrm{H}, \mathrm{ddd}, J=9.8,4.6\right.$, $2.3 \mathrm{~Hz}, \mathrm{H} 2), 3.64$ (1H, dd, $J=10.3,5.2 \mathrm{~Hz}, \mathrm{H} 6), 3.82$ (1H, dd, $J=11.5,7.5 \mathrm{~Hz}, \mathrm{H} 11 \mathrm{a}), 3.97$ (1H, dd, $J=12.6,2.3 \mathrm{~Hz}, \mathrm{H1a}), 4.24$ (1H, dd, $J=11.5,4.6 \mathrm{~Hz}, \mathrm{H} 11 \mathrm{~b}), 4.33$ (1H, dd, $J=12.6$, $4.6 \mathrm{~Hz}, \mathrm{H} 1 \mathrm{~b}), 4.95(1 \mathrm{H}, \mathrm{d}, J=5.2 \mathrm{~Hz}, \mathrm{H} 7), 4.98(1 \mathrm{H}, \mathrm{dd}, J=9.8,9.2 \mathrm{~Hz}, \mathrm{H} 3), 5.14(1 \mathrm{H}, \mathrm{dd}, J$ $=9.2,9.2 \mathrm{~Hz}, \mathrm{H} 4), 5.23-5.29(3 \mathrm{H}, \mathrm{m}, \mathrm{H} 5, \mathrm{H} 9, \mathrm{H} 10), 5.62(1 \mathrm{H}, \mathrm{dd}, J=9.8,1.2 \mathrm{~Hz}, \mathrm{H} 8) ;{ }^{13} \mathrm{C}\left\{{ }^{1} \mathrm{H}\right\}$ NMR (125 MHz, $\left.\mathrm{CDCl}_{3}\right) \delta 20.51,20.55$ (2C), 20.61, 20.64, 20.71, 20.72, 20.84, 20.87, 61.8, 62.2, 66.1, 67.3, 67.72, 67.74, 67.84, 69.1, 74.1, 76.3, 77.6, 169.2, 169.3, 169.6, 169.7, 170.29, 170.38, 170.45, 170.48, 170.54; HRMS (ESI-TOF) $[\mathrm{M}+\mathrm{Na}]^{+} \mathrm{m} / \mathrm{z}$ : Calcd for $\mathrm{C}_{29} \mathrm{H}_{40} \mathrm{O}_{19} \mathrm{Na}$ 715.2056; Found 715.2060.

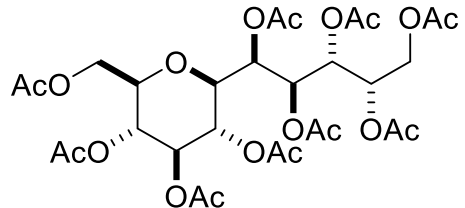

2

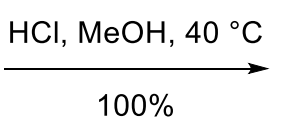
A solution of $2(12.3 \mathrm{mg}, 17.8 \mu \mathrm{mol})$ in $10 \mathrm{wt} \% \mathrm{HCl}$ in $\mathrm{MeOH}(1.78 \mathrm{~mL})$ was stirred at $40{ }^{\circ} \mathrm{C}$ for $2 \mathrm{~h}$. The reaction mixture was concentrated to afford diospyrodin $1(5.60 \mathrm{mg}, 17.8 \mu \mathrm{mol})$ in $100 \%$ yield: colorless oil; $[\alpha]_{\mathrm{D}}{ }^{24}+3.4(c 0.28$, $\mathrm{H}_{2} \mathrm{O}$ ); IR (film) 3357, 2927, 1648, 1420, 1318, 1089, $906 \mathrm{~cm}^{-1} ;{ }^{1} \mathrm{H}$ NMR $\left(400 \mathrm{MHz}, \mathrm{D}_{2} \mathrm{O}\right) \delta$ 
3.37 (2H, m, H2, H6), 3.50-3.58 (2H, m, H4, H7), 3.55 (1H, m, H3), 3.66 (1H, m, H5), 3.67

(1H, m, H11a), 3.69 (1H, m, H11b), 3.72 (1H, m, H1a), 3.89 (1H, d, J=12.4 Hz, H1b), 3.92

$(1 \mathrm{H}, \mathrm{d}, J=9.6 \mathrm{~Hz}, \mathrm{H} 9), 3.97(1 \mathrm{H}, \mathrm{d}, J=7.3 \mathrm{~Hz}, \mathrm{H} 10), 4.17(1 \mathrm{H}, \mathrm{d}, J=3.2 \mathrm{~Hz}, \mathrm{H} 8) ;{ }^{13} \mathrm{C}\left\{{ }^{1} \mathrm{H}\right\}$

NMR (100 MHz, D 20$) \delta 61.5,63.7,69.1,69.5,70.1,70.2,70.6,72.1,78.0,80.2,80.3$; HRMS

(ESI-TOF) $[\mathrm{M}+\mathrm{Na}]^{+} \mathrm{m} / \mathrm{z}$ : Calcd for $\mathrm{C}_{11} \mathrm{H}_{22} \mathrm{O}_{10} \mathrm{Na} 337.1105$; Found 337.1104 .
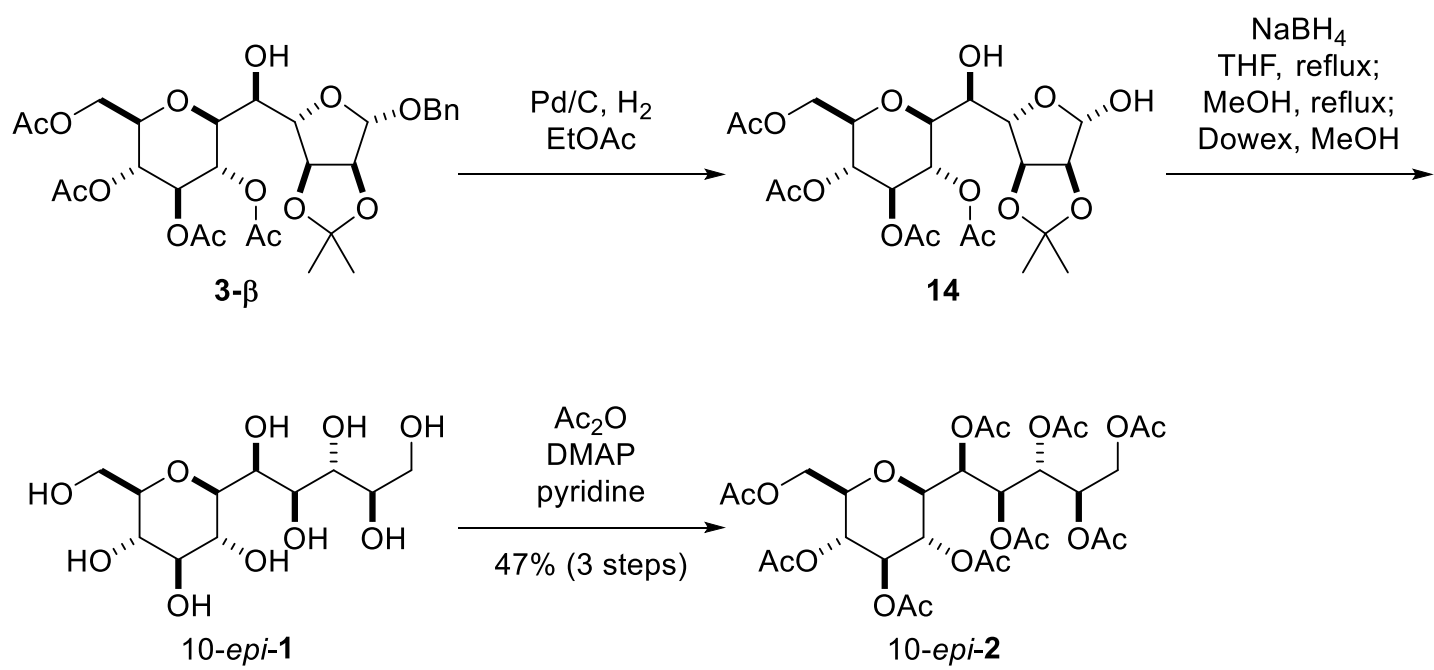

10-Epi-diospyrodin nonaacetate (10-epi-2). A suspension of benzyl acetal 3- $\boldsymbol{\beta}$ (26.2 $\mathrm{mg}$, $42.9 \mu \mathrm{mol})$ and $\mathrm{Pd} / \mathrm{C}(10 \mathrm{wt} \% \mathrm{Pd}$ on carbon, $5.2 \mathrm{mg})$ in EtOAc $(858 \mu \mathrm{L})$ was stirred under $\mathrm{H}_{2}$ atmosphere $(1 \mathrm{~atm})$ at $25^{\circ} \mathrm{C}$ for $16 \mathrm{~h}$. The reaction mixture was then filtered through a pad of Celite $(1 \mathrm{~g})$ with EtOAc. The filtrate was concentrated to afford the crude hemiacetal 14 (21.7 $\mathrm{mg}$ ), which was used in the next reaction without further purification.

$\mathrm{MeOH}(278 \mu \mathrm{L})$ was added over $1 \mathrm{~h}$ to a refluxing solution of the above crude 14 and $\mathrm{NaBH}_{4}$ (32.5 mg, $859 \mu \mathrm{mol})$ in THF $(4.29 \mathrm{~mL})$. After the reaction mixture was heated to reflux for 2 h, Dowex 50W (200-400 mesh, $550 \mathrm{mg})$ and $\mathrm{MeOH}(2.0 \mathrm{~mL})$ were added at $0{ }^{\circ} \mathrm{C}$. After being stirred at $25{ }^{\circ} \mathrm{C}$ for $16 \mathrm{~h}$, the reaction mixture was filtered, concentrated, and azeotroped with $\mathrm{MeOH}(2 \mathrm{~mL} \mathrm{x}$ ) to afford the crude 10-epi-1 (14.2 mg), which was used in the next reaction without further purification.

DMAP $(5.2 \mathrm{mg}, 43 \mu \mathrm{mol})$ and $\mathrm{Ac}_{2} \mathrm{O}(203 \mu \mathrm{L}, 2.15 \mathrm{mmol})$ were added to a solution of the above crude 10 -epi-1 in pyridine $(2.15 \mathrm{~mL})$ at $25{ }^{\circ} \mathrm{C}$. After the reaction mixture was stirred for 14 h, $\mathrm{H}_{2} \mathrm{O}(2 \mathrm{~mL})$ was added at $0{ }^{\circ} \mathrm{C}$. The resultant mixture was extracted with $\mathrm{CHCl}_{3}(3 \mathrm{~mL} \times 3)$. The combined organic layers were washed with brine $(10 \mathrm{~mL})$, dried over $\mathrm{Na}_{2} \mathrm{SO}_{4}$, filtered, and concentrated. The residue was purified by flash column chromatography on silica gel ( $2.5 \mathrm{~g}$, hexane $/ i$-PrOH $=3 / 1)$ to afford 10-epi-2 $(14.1 \mathrm{mg}, 20.4 \mu \mathrm{mol})$ in 47\% yield over 3 steps: 
colorless oil; $[\alpha]_{\mathrm{D}}^{25}+7.2\left(c 0.71, \mathrm{CHCl}_{3}\right)$; IR (film) 2925, 1750, 1433, 1372, 1221, 1041, 960 $\mathrm{cm}^{-1} ;{ }^{1} \mathrm{H}$ NMR $\left(500 \mathrm{MHz}, \mathrm{CDCl}_{3}\right) \delta 2.00\left(6 \mathrm{H}, \mathrm{s}, \mathrm{CH}_{3} \mathrm{CO}\right.$ x2), $2.03\left(3 \mathrm{H}, \mathrm{s}, \mathrm{CH} \mathrm{CO}_{3}\right), 2.04(3 \mathrm{H}$, s, $\left.\mathrm{CH}_{3} \mathrm{CO}\right), 2.06\left(3 \mathrm{H}, \mathrm{s}, \mathrm{CH}_{3} \mathrm{CO}\right), 2.07\left(6 \mathrm{H}, \mathrm{s}, \mathrm{CH}_{3} \mathrm{CO} \times 2\right), 2.09\left(3 \mathrm{H}, \mathrm{s}, \mathrm{CH}_{3} \mathrm{CO}\right), 2.15(3 \mathrm{H}, \mathrm{s}$, $\left.\mathrm{CH}_{3} \mathrm{CO}\right), 3.55$ (1H, ddd, $\left.J=10.1,4.9,2.0 \mathrm{~Hz}, \mathrm{H} 2\right), 3.66(1 \mathrm{H}, \mathrm{dd}, J=9.8,4.3 \mathrm{~Hz}, \mathrm{H} 6), 4.01$ $(1 \mathrm{H}, \mathrm{dd}, J=12.6,2.0 \mathrm{~Hz}, \mathrm{H} 1 \mathrm{a}), 4.17(1 \mathrm{H}, \mathrm{dd}, J=12.4,7.6 \mathrm{~Hz}, \mathrm{H} 11 \mathrm{a}), 4.31$ (1H, dd, $J=12.6$, $4.9 \mathrm{~Hz}, \mathrm{H} 1 \mathrm{~b}), 4.34$ (1H, dd, $J=12.4,3.2 \mathrm{~Hz}, \mathrm{H} 11 \mathrm{~b}), 5.01$ (1H, dd, $J=10.1,8.9 \mathrm{~Hz}, \mathrm{H} 3), 5.12$ $(1 \mathrm{H}, \mathrm{dd}, J=4.3,2.6 \mathrm{~Hz}, \mathrm{H} 7), 5.16(1 \mathrm{H}, \mathrm{dd}, J=8.9,8.9 \mathrm{~Hz}, \mathrm{H} 4), 5.18-5.22$ (3H, m, H5, H9, $\mathrm{H} 10), 5.62(1 \mathrm{H}, \mathrm{dd}, J=8.3,2.6 \mathrm{~Hz}, \mathrm{H} 8) ;{ }^{13} \mathrm{C}\left\{{ }^{1} \mathrm{H}\right\}$ NMR (125 MHz, $\left.\mathrm{CDCl}_{3}\right) \delta 20.57$ (2C), 20.61 (2C), 20.71 (2C), 20.76, 20.79, 20.9, 61.6, 61.9, 67.6, 67.9, 68.6 (2C), 69.4, 69.9, 74.1, 76.2, 76.9 (deduced from HMQC), 169.2, 169.3, 169.4, 169.6, 170.1 (2C), 170.3, 170.60, 170.61; HRMS (ESI-TOF) m/z: Calcd for $\mathrm{C}_{29} \mathrm{H}_{40} \mathrm{O}_{19} \mathrm{Na}[\mathrm{M}+\mathrm{Na}]^{+}$715.2056; Found 715.2075.
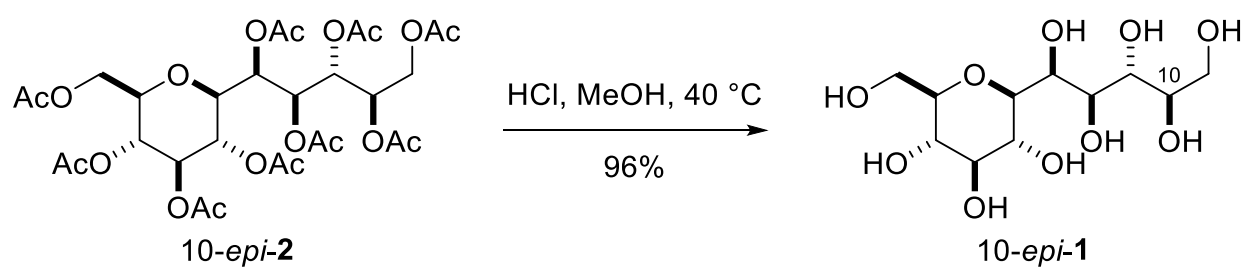

10-Epi-diospyrodin (10-epi-1). A solution of 10-epi-2 (14.1 mg, $20.4 \mu \mathrm{mol})$ in $10 \mathrm{wt} \% \mathrm{HCl}$ in $\mathrm{MeOH}(2.04 \mathrm{~mL})$ was stirred at $40{ }^{\circ} \mathrm{C}$ for $2 \mathrm{~h}$. The reaction mixture was concentrated to afford 10-epi-1 $(6.15 \mathrm{mg}, 19.6 \mu \mathrm{mol})$ in $96 \%$ yield: colorless oil; $[\alpha]_{\mathrm{D}}{ }^{24}+0.78\left(c 0.31, \mathrm{H}_{2} \mathrm{O}\right)$; IR (film) 3288, 2910, 1645, 1359, 1244, 1037, $879 \mathrm{~cm}^{-1} ;{ }^{1} \mathrm{H}$ NMR (400 MHz, $\left.\mathrm{D}_{2} \mathrm{O}\right) \delta 3.37-3.40$ (2H, m), 3.48-3.54 (3H, m), 3.65-3.72 (2H, m), 3.77-3.84 (2H, m), 3.86-3.95 (3H, m), $4.13(1 \mathrm{H}$, $\mathrm{d}, J=3.2 \mathrm{~Hz}) ;{ }^{13} \mathrm{C}\left\{{ }^{1} \mathrm{H}\right\} \mathrm{NMR}\left(100 \mathrm{MHz}, \mathrm{D}_{2} \mathrm{O}\right) \delta 61.5,62.4,70.0,70.4,70.7,71.7,72.1,73.2$, 78.0, 79.8, 80.2; HRMS (ESI-TOF) $[\mathrm{M}+\mathrm{Na}]^{+} \mathrm{m} / \mathrm{z}$ : Calcd for $\mathrm{C}_{11} \mathrm{H}_{22} \mathrm{O}_{10} \mathrm{Na}$ 337.1105; Found 337.1105 . 

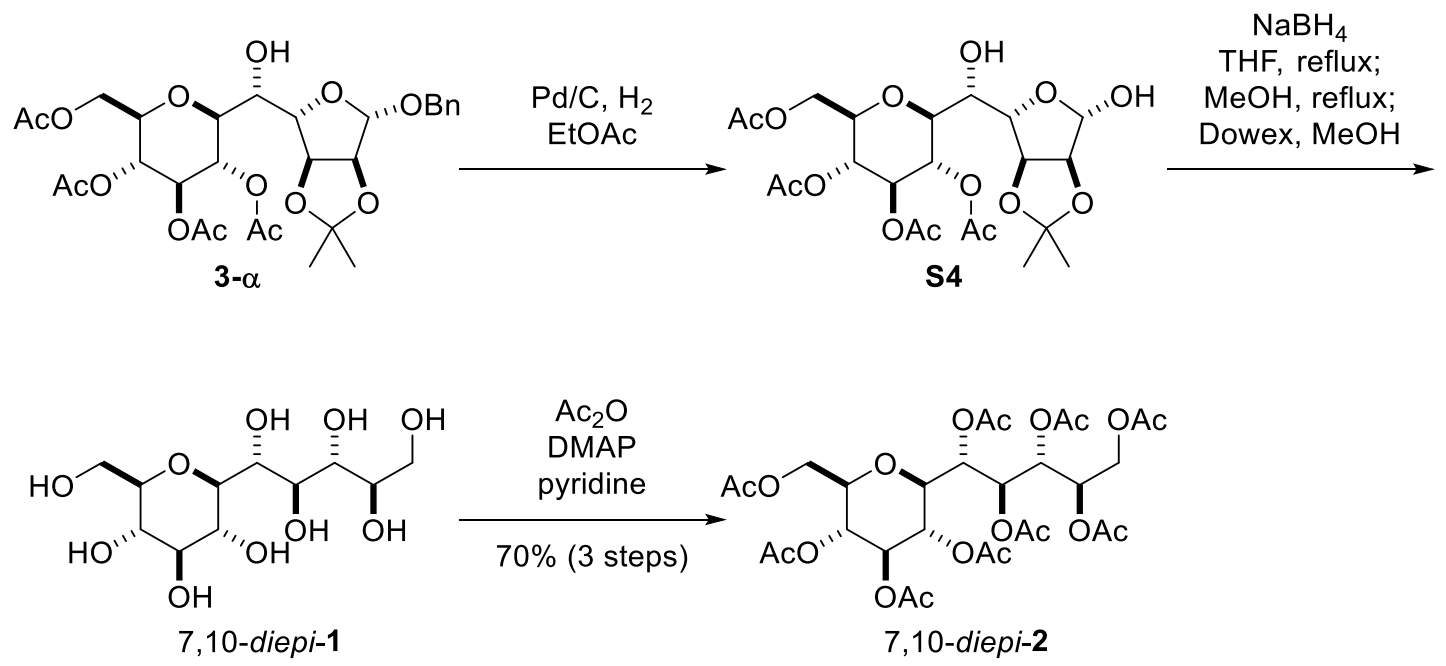

7,10-Diepi-diospyrodin nonaacetate (7,10-diepi-2). A suspension of benzyl acetal 3- $\alpha(22.9$ $\mathrm{mg}, 37.5 \mu \mathrm{mol})$ and $\mathrm{Pd} / \mathrm{C}(10 \mathrm{wt} \% \mathrm{Pd}$ on carbon, $4.6 \mathrm{mg})$ in EtOAc $(750 \mu \mathrm{L})$ was stirred under $\mathrm{H}_{2}$ atmosphere $(1 \mathrm{~atm})$ at $25^{\circ} \mathrm{C}$ for $17 \mathrm{~h}$. The reaction mixture was then filtered through a pad of Celite (1 g) with EtOAc. The filtrate was concentrated to afford the crude hemiacetal S4 $(18.5 \mathrm{mg}$ ), which was used in the next reaction without further purification. HRMS (ESITOF) $[\mathrm{M}+\mathrm{Na}]^{+} \mathrm{m} / \mathrm{z}$ : Calcd for $\mathrm{C}_{22} \mathrm{H}_{32} \mathrm{O}_{14} \mathrm{Na}$ 543.1684; Found 543.1663.

$\mathrm{MeOH}(250 \mu \mathrm{L})$ was added over $1 \mathrm{~h}$ to a refluxing solution of the above crude $\mathbf{S 3}$ and $\mathrm{NaBH}_{4}$ (28.4 mg, $751 \mu \mathrm{mol})$ in THF $(3.75 \mathrm{~mL})$. After the reaction mixture was heated to reflux for 2 h, Dowex 50W (200-400 mesh, $500 \mathrm{mg})$ and $\mathrm{MeOH}(2.0 \mathrm{~mL})$ were added at $0{ }^{\circ} \mathrm{C}$. After being stirred at $25{ }^{\circ} \mathrm{C}$ for $16 \mathrm{~h}$, the reaction mixture was filtered, concentrated, and azeotroped with $\mathrm{MeOH}(2 \mathrm{~mL} \times 3)$ to afford the crude 7,10-diepi-1 $(12.3 \mathrm{mg})$, which was used in the next reaction without further purification.

DMAP (4.6 mg, $38 \mu \mathrm{mol})$ and $\mathrm{Ac}_{2} \mathrm{O}(177 \mu \mathrm{L}, 1.87 \mathrm{mmol})$ were added to a solution of the above crude 7,10 -diepi-1 in pyridine $(1.88 \mathrm{~mL})$ at $25^{\circ} \mathrm{C}$. After the reaction mixture was stirred for $14 \mathrm{~h}, \mathrm{H}_{2} \mathrm{O}(2 \mathrm{~mL})$ was added at $0{ }^{\circ} \mathrm{C}$. The resultant mixture was extracted with $\mathrm{CHCl}_{3}(3 \mathrm{~mL}$ $\mathrm{x} 3)$. The combined organic layers were washed with brine $(10 \mathrm{~mL})$, dried over $\mathrm{Na}_{2} \mathrm{SO}_{4}$, filtered, and concentrated. The residue was purified by flash column chromatography on silica gel $(1.5 \mathrm{~g}$, hexane $/ \mathrm{i}$-PrOH $=5 / 1)$ to afford 7,10-diepi-2 $(18.3 \mathrm{mg}, 26.4 \mu \mathrm{mol})$ in $70 \%$ yield over 3 steps: colorless oil; $[\alpha]_{\mathrm{D}}{ }^{24}+17.8$ (c 0.92, $\mathrm{CHCl}_{3}$ ); IR (film) 2961, 1749, 1433, 1372, 1221, 1038, 905, $737 \mathrm{~cm}^{-1}$; ${ }^{1} \mathrm{H}$ NMR (500 MHz, $\left.\mathrm{CDCl}_{3}\right) \delta 1.97\left(3 \mathrm{H}, \mathrm{s}, \mathrm{CH} \mathrm{COO}_{3}\right), 2.00(3 \mathrm{H}, \mathrm{s}$, $\left.\mathrm{CH}_{3} \mathrm{CO}\right), 2.02\left(3 \mathrm{H}, \mathrm{s}, \mathrm{CH}_{3} \mathrm{CO}\right), 2.03\left(3 \mathrm{H}, \mathrm{s}, \mathrm{CH} \mathrm{H}_{3} \mathrm{CO}\right), 2.06\left(3 \mathrm{H}, \mathrm{s}, \mathrm{CH} \mathrm{H}_{3} \mathrm{CO}\right), 2.08(3 \mathrm{H}, \mathrm{s}$, $\left.\mathrm{CH}_{3} \mathrm{CO}\right), 2.09\left(6 \mathrm{H}, \mathrm{s}, \mathrm{CH}_{3} \mathrm{CO} \times 2\right), 2.15\left(3 \mathrm{H}, \mathrm{s}, \mathrm{CH}_{3} \mathrm{CO}\right), 3.56(1 \mathrm{H}, \mathrm{ddd}, J=9.8,5.0,2.3 \mathrm{~Hz}$, H2), 3.64 (1H, dd, $J=9.6,1.7$ Hz, H6), 4.04-4.08 (2H, m, H1a, H11a), 4.15 (1H, dd, $J=12.6$, 
$5.0 \mathrm{~Hz}, \mathrm{H} 1 \mathrm{~b}), 4.41$ (1H, dd, $J=12.6,2.9 \mathrm{~Hz}, \mathrm{H} 11 \mathrm{~b}), 4.93$ (1H, dd, $J=9.8,9.6 \mathrm{~Hz}, \mathrm{H} 5), 5.02$ $(1 \mathrm{H}, \mathrm{dd}, J=9.8,9.8 \mathrm{~Hz}, \mathrm{H} 3), 5.15(1 \mathrm{H}, \mathrm{dd}, J=9.8,9.8 \mathrm{~Hz}, \mathrm{H} 4), 5.27$ (1H, ddd, $J=6.3,6.3$, $2.9 \mathrm{~Hz}, \mathrm{H} 10), 5.31-5.33$ (2H, m, H7, H9), 5.41 (1H, dd, $J=8.6,2.3 \mathrm{~Hz}, \mathrm{H} 8) ;{ }^{13} \mathrm{C}\left\{{ }^{1} \mathrm{H}\right\} \mathrm{NMR}$ $\left(125 \mathrm{MHz}, \mathrm{CDCl}_{3}\right) \delta 20.54$ (2C), 20.58, 20.62 (2C), 20.64 (2C), 20.68, 20.75, 62.0, 62.5, 66.6, $67.0,68.4,69.3,69.7,70.1,74.5,75.6,76.6,168.8,169.37,169.44,169.54,169.56,170.1$, 170.4, 170.6, 170.8; HRMS (ESI-TOF) $[\mathrm{M}+\mathrm{Na}]^{+} \mathrm{m} / \mathrm{z}$ : Calcd for $\mathrm{C}_{29} \mathrm{H}_{40} \mathrm{O}_{19} \mathrm{Na}$ 715.2056; Found 715.2033 .
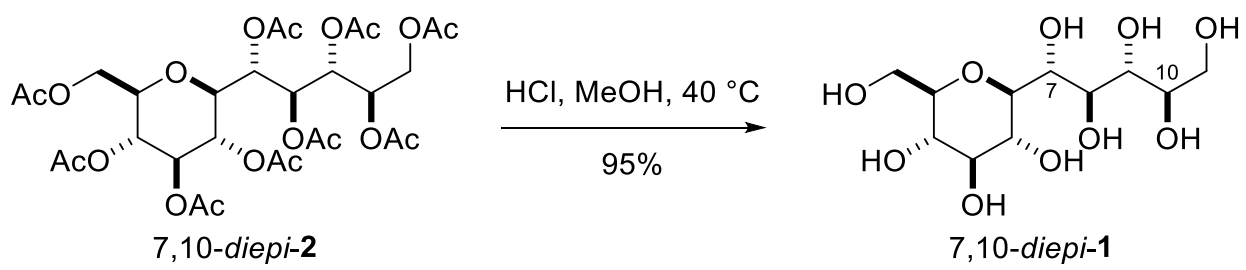

7,10-Diepi-diospyrodin (7,10-diepi-1). A solution of 7,10-diepi-2 $(18.3 \mathrm{mg}, 26.4 \mu \mathrm{mol})$ in $10 \mathrm{wt} \% \mathrm{HCl}$ in $\mathrm{MeOH}(2.64 \mathrm{~mL})$ was stirred at $40{ }^{\circ} \mathrm{C}$ for $2 \mathrm{~h}$. The reaction mixture was concentrated to afford 7,10-diepi-1 $(7.88 \mathrm{mg}, 25.1 \mu \mathrm{mol})$ in $95 \%$ yield: colorless oil; $[\alpha]_{\mathrm{D}}{ }^{24}$ 4.0 (c 0.39, $\mathrm{H}_{2} \mathrm{O}$ ); IR (film) 3359, 2929, 1648, 1423, 1255, 1085, 1047, $894 \mathrm{~cm}^{-1}$; ${ }^{1} \mathrm{H}$ NMR (400 MHz, $\left.\mathrm{D}_{2} \mathrm{O}\right) \delta 3.33-3.42(2 \mathrm{H}, \mathrm{m}), 3.49-3.60(3 \mathrm{H}, \mathrm{m}), 3.65(1 \mathrm{H}, \mathrm{dd}, J=11.9,6.9 \mathrm{~Hz}), 3.71(1 \mathrm{H}$, $\mathrm{dd}, J=12.4,5.5 \mathrm{~Hz}), 3.82(1 \mathrm{H}, \mathrm{dd}, J=11.9,3.2 \mathrm{~Hz}), 3.86-3.91(2 \mathrm{H}, \mathrm{m}), 4.06(1 \mathrm{H}, \mathrm{d}, J=8.7$ $\mathrm{Hz}) ;{ }^{13} \mathrm{C}\left\{{ }^{1} \mathrm{H}\right\} \mathrm{NMR}\left(100 \mathrm{MHz}, \mathrm{D}_{2} \mathrm{O}\right) \delta 61.5,63.2,68.8,69.4,70.2,71.2,72.3,73.8,77.7,78.1$, 79.8; HRMS (ESI-TOF) $[\mathrm{M}+\mathrm{Na}]^{+} \mathrm{m} / \mathrm{z}$ : Calcd for $\mathrm{C}_{11} \mathrm{H}_{22} \mathrm{O}_{10} \mathrm{Na} 337.1105$; Found 337.1100.

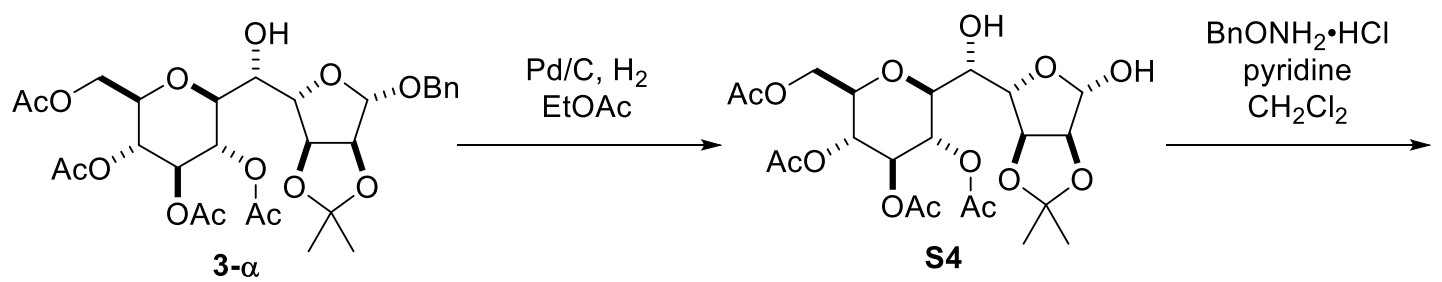

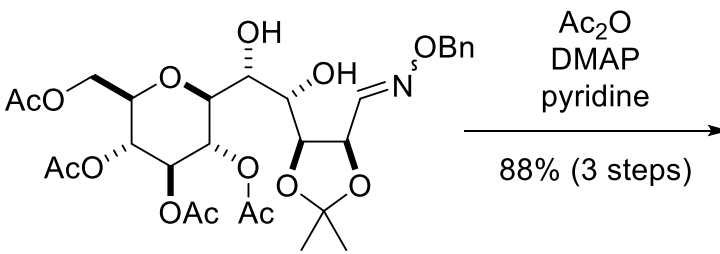

S5

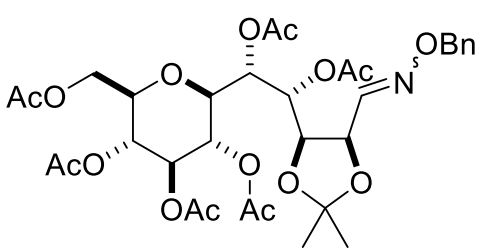

S6 $(E / Z=1: 0.3)$

Oxime S6. A suspension of benzyl acetal 3- $\alpha(86.9 \mathrm{mg}, 142 \mu \mathrm{mol})$ and $\mathrm{Pd} / \mathrm{C}(10 \mathrm{wt} \% \mathrm{Pd}$ on carbon, $17.4 \mathrm{mg})$ in EtOAc $(2.84 \mathrm{~mL})$ was stirred under $\mathrm{H}_{2}$ atmosphere $(1 \mathrm{~atm})$ at $25^{\circ} \mathrm{C}$ for 12 
h. The reaction mixture was then filtered through a pad of Celite $(2 \mathrm{~g})$ with EtOAc. The filtrate was concentrated to afford the crude hemiacetal $\mathbf{S 4}(73.8 \mathrm{mg})$, which was used in the next reaction without further purification.

$\mathrm{BnONH}_{2} \cdot \mathrm{HCl}(68.0 \mathrm{mg}, 426 \mu \mathrm{mol})$ was added to a solution of the above crude $\mathbf{S 4}$ in $\mathrm{CH}_{2} \mathrm{Cl}_{2}$ $(568 \mu \mathrm{L})$ and pyridine $(75.7 \mu \mathrm{L})$ at $25{ }^{\circ} \mathrm{C}$. After being stirred at $25{ }^{\circ} \mathrm{C}$ for $2 \mathrm{~d}$, the reaction mixture was concentrated. The residue was filtered through a pad of silica gel $(1 \mathrm{~g}$, EtOAc) to afford the crude $\mathbf{S 5}(93.1 \mathrm{mg}$ ), which was used in the next reaction without further purification.

DMAP $(1.7 \mathrm{mg}, 14 \mu \mathrm{mol})$ and $\mathrm{Ac}_{2} \mathrm{O}(268 \mu \mathrm{L}, 2.84 \mathrm{mmol})$ were added to a solution of the above crude $\mathbf{S 5}$ in pyridine $(14.2 \mathrm{~mL})$ at $25^{\circ} \mathrm{C}$. After being stirred for $14 \mathrm{~h}$, the reaction mixture was diluted with toluene $(15 \mathrm{~mL})$ and concentrated. The residue was purified by flash column chromatography on silica gel $(5 \mathrm{~g}$, hexane/EtOAc $=4 / 1$ to $1 / 1)$ to afford a $1: 0.3$ diastereomeric mixture of oxime $\mathbf{S 6}(88.5 \mathrm{mg}, 125 \mu \mathrm{mol})$ in $88 \%$ yield over 3 steps: colorless oil; IR (film) 3648, 3482, 2987, 2940, 2882, 2109, 1747, 1371, 1230, $1037 \mathrm{~cm}^{-1}$; ${ }^{1} \mathrm{H}$ NMR (400 MHz, $\mathrm{C}_{6} \mathrm{D}_{6}$ ) $\delta 1.12\left(3 \mathrm{H} \mathrm{x} 3 / 13, \mathrm{~s}, \mathrm{CH}_{3}\right.$ of acetonide), 1.19 (3H x10/13, s, $\mathrm{CH}_{3}$ of acetonide), 1.32 (3H x10/13, s, $\mathrm{CH}_{3}$ of acetonide), $1.46\left(3 \mathrm{H} \mathrm{x} 3 / 13, \mathrm{~s}, \mathrm{CH}_{3}\right.$ of acetonide), $1.65\left(3 \mathrm{H} \mathrm{x10/13,} \mathrm{s,} \mathrm{CH}_{3} \mathrm{CO}\right), 1.67$ (3H x10/13, s, $\left.\mathrm{CH}_{3} \mathrm{CO}\right), 1.71\left(3 \mathrm{H}, \mathrm{s}, \mathrm{CH}_{3} \mathrm{CO}\right), 1.74(6 \mathrm{H}, \mathrm{s}, \mathrm{CH} 3 \mathrm{CO} \times 2), 1.77(3 \mathrm{H} \mathrm{x} 3 / 13, \mathrm{~s}$, $\left.\mathrm{CH}_{3} \mathrm{CO}\right), 1.79\left(3 \mathrm{H} \times 10 / 13, \mathrm{~s}, \mathrm{CH}_{3} \mathrm{CO}\right), 1.85\left(3 \mathrm{H} \times 3 / 13, \mathrm{~s}, \mathrm{CH}_{3} \mathrm{CO}\right), 1.86\left(3 \mathrm{H} \times 10 / 13, \mathrm{~s}, \mathrm{CH}_{3} \mathrm{CO}\right)$, $3.16(1 \mathrm{H} \mathrm{x} 3 / 13, \mathrm{~m}, \mathrm{H} 2), 3.24$ (1H x10/13, m, H2), 3.67 (1H x10/13, m, H6), 3.82 (1H x3/13, d, $J=7.8 \mathrm{~Hz}, \mathrm{H6}$ ), 4.08-4.14 (1H, m, H1a), 4.22-4.28 (1H, m, H1b), 4.50-4.56 (1H, m, H9), 4.87 $(1 \mathrm{H} \mathrm{x} 10 / 13, \mathrm{dd}, J=8.7,6.0 \mathrm{~Hz}, \mathrm{H} 10), 5.01\left(2 \mathrm{H} \mathrm{x10/13,} \mathrm{s,} \mathrm{OCH}_{2} \mathrm{Ph}\right), 5.06(2 \mathrm{H} \mathrm{x10/13,} \mathrm{s,}$ $\left.\mathrm{OCH}_{2} \mathrm{Ph}\right), 5.23-5.34(1 \mathrm{H}, \mathrm{m}, \mathrm{H} 3), 5.36-5.43$ (3H x3/13 and $2 \mathrm{H} \mathrm{x10/13,} \mathrm{m}, \mathrm{H} 4, \mathrm{H} 5, \mathrm{H} 10$ and H4, H5), 5.55-5.57 (1H, m, H7), 5.70-5.77 (1H, m, H8), 6.99 (1H x3/13, d, $J=6.0 \mathrm{~Hz}, \mathrm{H} 11)$, 7.07-7.32 (5H, m, aromatic), $7.64(1 \mathrm{H} \mathrm{x10/13,} \mathrm{d,} J=8.7 \mathrm{~Hz}, \mathrm{H} 11)$; Detectable signals of ${ }^{13} \mathrm{C}\left\{{ }^{1} \mathrm{H}\right\} \mathrm{NMR}\left(100 \mathrm{MHz}, \mathrm{C}_{6} \mathrm{D}_{6}\right), \delta 20.07,20.11,20.21,20.23,20.30,20.35,20.46,20.49,20.7$, 25.3, 25.4, 27.7, 28.0, 61.9, 62.0, 67.6, 67.7, 67.9, 68.2, 68.5, 68.7, 69.4, 71.3, 74.9, 75.0, 76.0, 76.3, 76.4, 76.5, 76.7, 76.96, 76.98, 77.1, 109.8, 109.9, 128.1, 128.5, 128.6, 128.71, 128.74, 137.6, 137.9, 147.3, 148.0, 169.00, 169.05, 169.08, 169.17, 169.30, 169.34, 169.98, 170.02, 170.07, 170.12, 170.22, 170.4; HRMS (ESI-TOF) $[\mathrm{M}+\mathrm{Na}]^{+} \mathrm{m} / \mathrm{z}$ : Calcd for $\mathrm{C}_{33} \mathrm{H}_{43} \mathrm{NO}_{16} \mathrm{Na}$ 732.2474; Found 732.2448. 

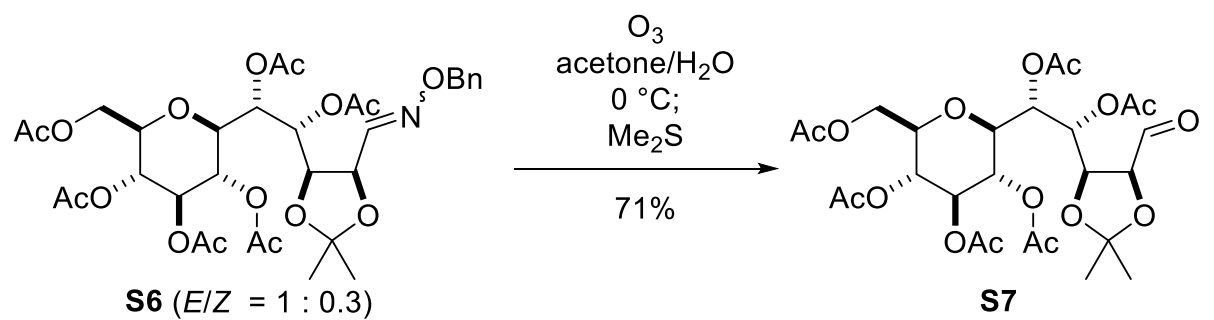

Aldehyde S7. $\mathrm{O}_{3}$ was bubbled into a solution of oxime $\mathbf{S 6}(32.3 \mathrm{mg}, 45.5 \mu \mathrm{mol})$ in acetone $(13.7 \mathrm{~mL})$ and $\mathrm{H}_{2} \mathrm{O}(1.52 \mathrm{~mL})$ for $1 \mathrm{~h}$ at $0{ }^{\circ} \mathrm{C}$. The excess $\mathrm{O}_{3}$ was removed by passing a stream of $\mathrm{Ar}$ at $25{ }^{\circ} \mathrm{C}$ for $10 \mathrm{~min}$, and then $\mathrm{Me}_{2} \mathrm{~S}(1.52 \mathrm{~mL}, 20.6 \mathrm{mmol})$ was added. The reaction mixture was warmed to $25{ }^{\circ} \mathrm{C}$ and stirred for $13 \mathrm{~h}$. The mixture was concentrated, and the residue was purified by flash column chromatography on silica gel $(2.5 \mathrm{~g}$, hexane/EtOAc $=2 / 1$ to $3 / 2$ ) to afford aldehyde $\mathbf{S 7}(19.6 \mathrm{mg}, 32.4 \mu \mathrm{mol})$ in $71 \%$ yield: colorless oil; $[\alpha]_{\mathrm{D}}{ }^{24}+35.7$ ( $c$ 0.89, $\mathrm{CHCl}_{3}$ ); IR (film) 3486, 2987, 2940, 1754, 1433, 1372, 1227, $1037 \mathrm{~cm}^{-1} ;{ }^{1} \mathrm{H}$ NMR (400 $\left.\mathrm{MHz}, \mathrm{C}_{6} \mathrm{D}_{6}\right) \delta 1.23\left(3 \mathrm{H}, \mathrm{s}, \mathrm{CH}_{3}\right.$ of acetonide), $1.44\left(3 \mathrm{H}, \mathrm{s}, \mathrm{CH}_{3}\right.$ of acetonide), $1.65(3 \mathrm{H}, \mathrm{s}$, $\left.\mathrm{CH}_{3} \mathrm{CO}\right), 1.66\left(3 \mathrm{H}, \mathrm{s}, \mathrm{CH}_{3} \mathrm{CO}\right), 1.70\left(3 \mathrm{H}, \mathrm{s}, \mathrm{CH}_{3} \mathrm{CO}\right), 1.74\left(3 \mathrm{H}, \mathrm{s}, \mathrm{CH}_{3} \mathrm{CO}\right), 1.83(3 \mathrm{H}, \mathrm{s}$, $\left.\mathrm{CH}_{3} \mathrm{CO}\right), 1.89\left(3 \mathrm{H}, \mathrm{s}, \mathrm{CH}_{3} \mathrm{CO}\right), 3.11(1 \mathrm{H}, \mathrm{ddd}, J=10.1,5.0,2.3, \mathrm{H} 2), 3.54(1 \mathrm{H}, \mathrm{dd}, J=10.1$, $2.3 \mathrm{~Hz}, \mathrm{H6}), 4.08$ (1H, dd, $J=12.4,2.3 \mathrm{~Hz}, \mathrm{H1a}), 4.15$ (1H, dd, $J=12.4,5.0 \mathrm{~Hz}, \mathrm{H} 1 \mathrm{~b}), 4.48$ $(1 \mathrm{H}, \mathrm{dd}, J=6.8,3.6 \mathrm{~Hz}, \mathrm{H10}) 4.77(1 \mathrm{H}, \mathrm{dd}, J=6.8,3.9 \mathrm{~Hz}, \mathrm{H} 9), 5.24(1 \mathrm{H}, \mathrm{dd}, J=10.1,9.6$ Hz, H3), 5.30 (1H, dd, $J=10.1,9.2 \mathrm{~Hz}, \mathrm{H} 5), 5.62(1 \mathrm{H}, \mathrm{dd}, J=9.6,9.2 \mathrm{~Hz}, \mathrm{H} 4), 5.64(1 \mathrm{H}, \mathrm{dd}$, $J=5.5,2.3 \mathrm{~Hz}, \mathrm{H} 7), 5.82(1 \mathrm{H}, \mathrm{dd}, J=5.5,2.8 \mathrm{~Hz}, \mathrm{H} 8), 9.66(1 \mathrm{H}, \mathrm{d}, J=3.6 \mathrm{~Hz}, \mathrm{H} 11) ;{ }^{13} \mathrm{C}\left\{{ }^{1} \mathrm{H}\right\}$ NMR (100 MHz, $\left.\mathrm{C}_{6} \mathrm{D}_{6}\right), \delta$ 20.05, 20.18, 20.23 (2C), 20.27, 20.7, 25.1, 27.5, 61.8, 67.4, 67.7, $68.5,70.8,74.7,76.8,76.9,79.1,81.3,110.1,168.7,169.1,169.5,169.9,170.1,170.5,198.4$; HRMS (ESI-TOF) $[\mathrm{M}+\mathrm{Na}]^{+} \mathrm{m} / \mathrm{z}$ : Calcd for $\mathrm{C}_{26} \mathrm{H}_{36} \mathrm{O}_{16} \mathrm{Na}$ 627.1896; Found 627.1868.
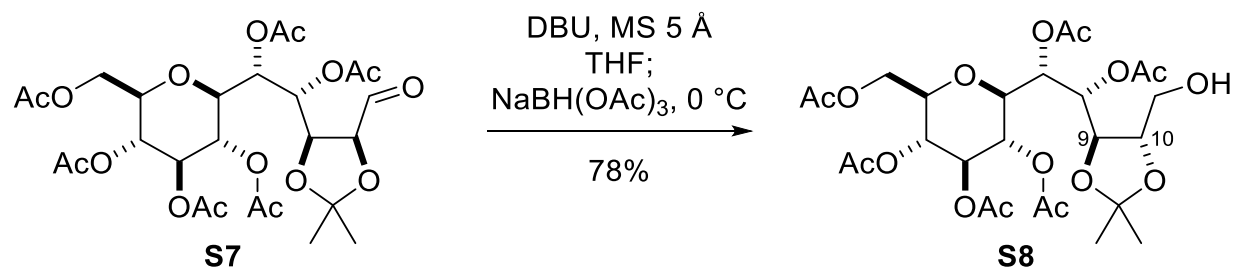

Alcohol S8. MS $5 \AA$ (160 mg) was added to a solution of aldehyde S7 (16.0 mg, $26.5 \mu \mathrm{mol})$ in THF $(2.65 \mathrm{~mL})$ at $25^{\circ} \mathrm{C}$. After the resultant mixture was stirred at $25^{\circ} \mathrm{C}$ for $2 \mathrm{~h}, \mathrm{DBU}(4.0$ $\mu \mathrm{L}, 27 \mu \mathrm{mol}$ ) was added. The reaction mixture was stirred at $25{ }^{\circ} \mathrm{C}$ for $16 \mathrm{~h}$, and then $\mathrm{NaBH}(\mathrm{OAc})_{3}(112 \mathrm{mg}, 528 \mu \mathrm{mol})$ was added at $0{ }^{\circ} \mathrm{C}$. The reaction mixture was stirred at $0{ }^{\circ} \mathrm{C}$ for $1 \mathrm{~h}$, and then saturated aqueous $\mathrm{NH}_{4} \mathrm{Cl}(3 \mathrm{~mL})$ was added. The resultant mixture was extracted with EtOAc (3 mL x3). The combined organic layers were washed with brine (10 
$\mathrm{mL}$ ), dried over $\mathrm{Na}_{2} \mathrm{SO}_{4}$, filtered, and concentrated. The residue was purified by flash column chromatography on silica gel $(2 \mathrm{~g}$, hexane/EtOAc $=1 / 1$ to $1 / 2)$ to afford alcohol $\mathbf{S 8}(12.5 \mathrm{mg}$, $20.6 \mu \mathrm{mol})$ in $78 \%$ yield: colorless oil; $[\alpha]_{\mathrm{D}}{ }^{27}+13.2\left(c 0.66, \mathrm{CHCl}_{3}\right)$; IR (film) 3713, 2987, 1750, 1432, 1371, 1224, 1037, $906 \mathrm{~cm}^{-1} ;{ }^{1} \mathrm{H}$ NMR (500 MHz, $\left.\mathrm{C}_{6} \mathrm{D}_{6}\right) \delta 1.326\left(3 \mathrm{H}, \mathrm{s}, \mathrm{CH}_{3}\right.$ of acetonide), $1.328\left(3 \mathrm{H}, \mathrm{s}, \mathrm{CH}_{3}\right.$ of acetonide), $1.63\left(3 \mathrm{H}, \mathrm{s}, \mathrm{CH}_{3} \mathrm{CO}\right), 1.70\left(3 \mathrm{H}, \mathrm{s}, \mathrm{CH}_{3} \mathrm{CO}\right), 1.73$ $\left(3 \mathrm{H}, \mathrm{s}, \mathrm{CH}_{3} \mathrm{CO}\right), 1.77\left(3 \mathrm{H}, \mathrm{s}, \mathrm{CH}_{3} \mathrm{CO}\right), 1.79\left(3 \mathrm{H}, \mathrm{s}, \mathrm{CH}_{3} \mathrm{CO}\right), 1.80\left(3 \mathrm{H}, \mathrm{s}, \mathrm{CH}_{3} \mathrm{CO}\right), 3.13(1 \mathrm{H}$, ddd, $J=9.8,5.5,2.3 \mathrm{~Hz}, \mathrm{H} 2), 3.43(1 \mathrm{H}, \mathrm{d}, J=9.8,1.2 \mathrm{~Hz}, \mathrm{H} 6), 3.66(1 \mathrm{H}, \mathrm{m}, \mathrm{H} 11 \mathrm{a}), 3.79(1 \mathrm{H}$, m, H11b), 4.10 (1H, dd, $J=12.3,2.3 \mathrm{~Hz}, \mathrm{H1a}), 4.17$ (1H, dd, $J=12.3,5.5 \mathrm{~Hz}, \mathrm{H} 1 \mathrm{~b}), 4.22(1 \mathrm{H}$, m, H10), 4.44 (1H, ddd, $J=7.5,2.3,2.3 \mathrm{~Hz}, \mathrm{H} 9), 5.20$ (1H, dd, $J=9.8,9.2 \mathrm{~Hz}, \mathrm{H} 3), 5.27(1 \mathrm{H}$, $\mathrm{dd}, J=9.8,9.2 \mathrm{~Hz}, \mathrm{H} 5), 5.32(1 \mathrm{H}, \mathrm{dd}, J=9.2,9.2 \mathrm{~Hz}, \mathrm{H} 4), 5.63-5.64(2 \mathrm{H}, \mathrm{m}, \mathrm{H} 7, \mathrm{H} 8) ;{ }^{13} \mathrm{C}\left\{{ }^{1} \mathrm{H}\right\}$ NMR (125 MHz, $\mathrm{C}_{6} \mathrm{D}_{6}$ ), $\delta$ 20.0, 20.18, 20.21, 20.32 (2C), 20.37, 26.9, 27.3, 62.0, 62.8, 67.5, 68.0, 68.6, 71.9, 74.8, 76.4, 76.81, 76.85, 79.1, 109.4, 169.0, 169.28, 169.34, 169.9, 170.07, 170.14; HRMS (ESI-TOF) [M+Na] $]^{+} \mathrm{m} / \mathrm{z}$ : Calcd for $\mathrm{C}_{26} \mathrm{H}_{38} \mathrm{O}_{16} \mathrm{Na} 629.2052$; Found 629.2022.

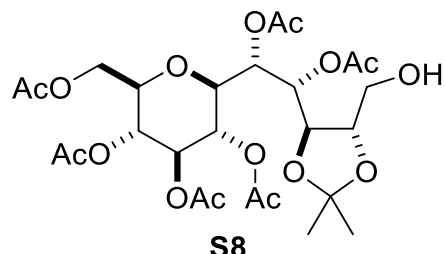

S8

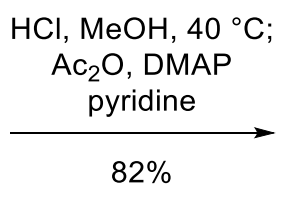

7-epi-2

7-Epi-diospyrodin nonaacetate (7-epi-2). A solution of alcohol S8 (17.1 mg, $28.2 \mu \mathrm{mol})$ in $0.5 \mathrm{M}$ solution of $\mathrm{HCl}$ in $\mathrm{MeOH}(2.82 \mathrm{~mL})$ was stirred at $40^{\circ} \mathrm{C}$ for $2 \mathrm{~h}$. The reaction mixture was concentrated, and the residue was dissolved in pyridine. DMAP (3.4 mg, $28 \mu \mathrm{mol})$ and $\mathrm{Ac}_{2} \mathrm{O}(133 \mu \mathrm{L}, 1.41 \mathrm{mmol})$ were successively added to the resultant solution at $0{ }^{\circ} \mathrm{C}$. After the reaction mixture was stirred at $25^{\circ} \mathrm{C}$ for $16 \mathrm{~h}, \mathrm{MeOH}(1 \mathrm{~mL})$ was added. The resultant mixture was concentrated and azeotroped with toluene $(1 \mathrm{~mL})$. The residue was purified by PTLC (hexane/EtOAc $=1 / 1)$ to afford 7-epi-2 $(16.0 \mathrm{mg}, 23.1 \mu \mathrm{mol})$ in 82\% yield: colorless oil; $[\alpha]_{\mathrm{D}}{ }^{25}-0.3\left(c 0.80, \mathrm{CHCl}_{3}\right)$; IR (film) 2958, 1750, 1434, 1372, 1223, 1038, $904 \mathrm{~cm}^{-1} ;{ }^{1} \mathrm{H}$ NMR $\left(500 \mathrm{MHz}, \mathrm{CDCl}_{3}\right) \delta 1.98\left(3 \mathrm{H}, \mathrm{s}, \mathrm{CH}_{3} \mathrm{CO}\right), 2.01\left(6 \mathrm{H}, \mathrm{s}, \mathrm{CH}_{3} \mathrm{CO} \times 2\right), 2.04\left(3 \mathrm{H}, \mathrm{s}, \mathrm{CH}_{3} \mathrm{CO}\right), 2.06$ $\left(6 \mathrm{H}, \mathrm{s}, \mathrm{CH}_{3} \mathrm{CO} \times 2\right), 2.07$ (3H, s, $\left.\mathrm{CH}_{3} \mathrm{CO}\right), 2.09$ (3H, s, $\left.\mathrm{CH}_{3} \mathrm{CO}\right), 2.13\left(3 \mathrm{H}, \mathrm{s}, \mathrm{CH}_{3} \mathrm{CO}\right), 3.58$ (1H, ddd, $J=9.7,5.7,2.3 \mathrm{~Hz}, \mathrm{H} 2), 3.65$ (1H, dd, $J=10.3,1.7 \mathrm{~Hz}, \mathrm{H} 6), 4.06-4.09$ (2H, m, H1a, H11a), 4.18-4.22 (2H, m, H1b, H11b), 4.94 (1H, dd, $J=10.3,9.8 \mathrm{~Hz}, \mathrm{H} 5), 5.02$ (1H, dd, $J=$ 9.8, $9.7 \mathrm{~Hz}, \mathrm{H} 3), 5.17$ (1H, dd, $J=9.8,9.8 \mathrm{~Hz}, \mathrm{H} 4), 5.25$ (1H, dd, $J=4.0,4.0 \mathrm{~Hz}, \mathrm{H} 9), 5.30$ $(1 \mathrm{H}, \mathrm{dd}, J=8.0,1.7 \mathrm{~Hz}, \mathrm{H} 7), 5.40(1 \mathrm{H}, \mathrm{dd}, J=8.0,4.0 \mathrm{~Hz}, \mathrm{H} 8), 4.51(1 \mathrm{H}, \mathrm{m}, \mathrm{H} 10) ;{ }^{13} \mathrm{C}\left\{{ }^{1} \mathrm{H}\right\}$ 
NMR (125 MHz, $\left.\mathrm{CDCl}_{3}\right) \delta 20.55$ (2C), 20.58, 20.60, 20.63 (3C), 20.66, 20.8, 62.2, 62.4, 66.8, $67.0,68.35,68.41,68.9,69.9,74.4,75.7,76.5,168.9,169.4,169.5,169.88,169.92,170.1$, 170.3, 170.4, 170.7; HRMS (ESI-TOF) $[\mathrm{M}+\mathrm{Na}]^{+} \mathrm{m} / \mathrm{z}$ : Calcd for $\mathrm{C}_{29} \mathrm{H}_{40} \mathrm{O}_{19} \mathrm{Na} 715.2056$; Found 715.2046 .
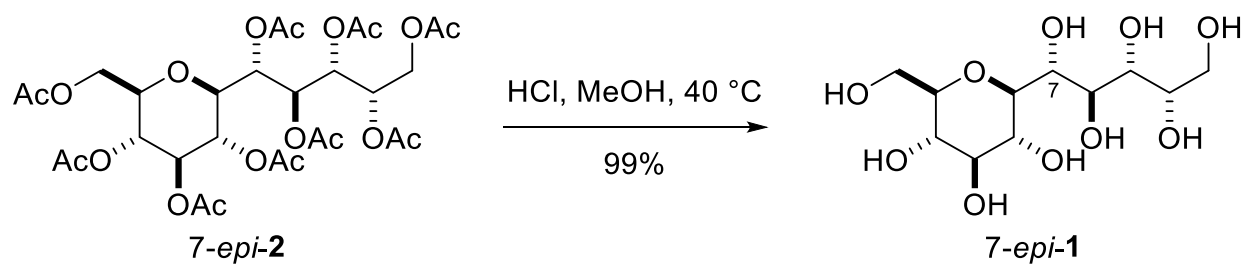

7-Epi-diospyrodin (7-epi-1). A solution of 7-epi-2 $(16.0 \mathrm{mg}, 23.1 \mu \mathrm{mol})$ in $10 \mathrm{wt} \% \mathrm{HCl}$ in $\mathrm{MeOH}(2.31 \mathrm{~mL})$ was stirred at $40^{\circ} \mathrm{C}$ for $2 \mathrm{~h}$. The reaction mixture was concentrated to afford 7-epi-1 (7.18 mg, $22.8 \mu \mathrm{mol})$ in 99\% yield: colorless oil; $[\alpha]_{\mathrm{D}}{ }^{24}-3.3\left(c 0.36, \mathrm{H}_{2} \mathrm{O}\right)$; IR (film) 3359, 2929, 1648, 1423, 1255, 1085, 1047, $894 \mathrm{~cm}^{-1}$; ${ }^{1} \mathrm{H}$ NMR (400 MHz, $\left.\mathrm{D}_{2} \mathrm{O}\right) \delta 3.33-3.44$ $(2 \mathrm{H}, \mathrm{m}), 3.54(1 \mathrm{H}, \mathrm{dd}, J=8.7,8.7 \mathrm{~Hz}), 3.57(1 \mathrm{H}, \mathrm{dd}, J=8.7,8.7 \mathrm{~Hz}), 3.61-3.74(4 \mathrm{H}, \mathrm{m}), 3.79$ $(1 \mathrm{H}, \mathrm{dd}, J=6.4,2.3 \mathrm{~Hz}), 3.87-3.94(2 \mathrm{H}, \mathrm{m}), 3.98-4.04(2 \mathrm{H}, \mathrm{m}) ;{ }^{13} \mathrm{C}\left\{{ }^{1} \mathrm{H}\right\} \mathrm{NMR}(100 \mathrm{MHz}$, $\left.\mathrm{D}_{2} \mathrm{O}\right) \delta 61.5,63.4,69.3,69.4,70.1,71.0,71.3,72.5,77.8,78.1,79.8$; HRMS (ESI-TOF) $[\mathrm{M}+\mathrm{Na}]^{+} \mathrm{m} / \mathrm{z}$ : Calcd for $\mathrm{C}_{11} \mathrm{H}_{22} \mathrm{O}_{10} \mathrm{Na} 337.1105$; Found 337.1110. 
2. Comparison of ${ }^{1} \mathrm{H}$ and ${ }^{13} \mathrm{C}\left\{{ }^{1} \mathrm{H}\right\}$ NMR data of the reported and synthetic diospyrodin nonaacetate (2) in $\mathrm{CDCl}_{3}$

Table S1. ${ }^{1} \mathrm{H}$ NMR data of the reported ${ }^{\mathrm{S} 3}$ and synthetic diospyrodin nonaacetate (2) in $\mathrm{CDCl}_{3}$

\begin{tabular}{|c|c|c|c|}
\hline \multirow[b]{2}{*}{ No. } & \multirow{2}{*}{$\begin{array}{c}\text { reported } 2(400 \mathrm{MHz})^{a} \\
{ }^{1} \mathrm{H}[\delta, \text { multi, } J(\mathrm{~Hz})]\end{array}$} & \multirow{2}{*}{$\begin{array}{l}\text { synthetic } 2(400 \mathrm{MHz}) \\
{ }^{1} \mathrm{H}[\delta, \text { multi, } J(\mathrm{~Hz})]\end{array}$} & \multirow[t]{2}{*}{$\Delta\left(\delta_{\text {reported }}-\delta_{\text {synthetic }}\right)$} \\
\hline & & & \\
\hline $1 \mathrm{a}$ & $3.97(\mathrm{dd}, 12.0,2.5)$ & $3.97(\mathrm{dd}, 12.6,2.3)$ & 0.00 \\
\hline $1 b$ & $4.33(\mathrm{dd}, 12.0,4.5)$ & $4.33(\mathrm{dd}, 12.6,4.6)$ & 0.00 \\
\hline 2 & $3.49(\mathrm{ddd}, 9.5,4.5,2.5)$ & $3.49(\mathrm{ddd}, 9.8,4.6,2.3)$ & 0.00 \\
\hline 3 & $4.97(\mathrm{dd}, 9.5,9.5)$ & $4.98(\mathrm{dd}, 9.8,9.2)$ & -0.01 \\
\hline 4 & $5.14(\mathrm{dd}, 9.5,9.5)$ & $5.14(\mathrm{dd}, 9.2,9.2)$ & 0.00 \\
\hline 5 & $5.27(\mathrm{dd}, 9.5,9.5)$ & $5.26(\mathrm{~m})$ & 0.01 \\
\hline 6 & $3.64(\mathrm{dd}, 9.5,4.5)$ & $3.64(\mathrm{dd}, 10.3,5.2)$ & 0.00 \\
\hline 7 & $4.95(\mathrm{dd}, 4.5,1.5)$ & $4.95(\mathrm{~d}, 5.2)$ & 0.00 \\
\hline 8 & $5.62(\mathrm{dd}, 4.5,1.5)$ & $5.62(\mathrm{dd}, 9.8,1.2)$ & 0.00 \\
\hline 9 & $5.24(\mathrm{dd}, 9.5,1.5)$ & $5.26(\mathrm{~m})$ & -0.02 \\
\hline 10 & $5.27(\mathrm{~m})$ & $5.26(\mathrm{~m})$ & 0.01 \\
\hline $11 \mathrm{a}$ & $3.82(\mathrm{dd}, 11.5,7.5)$ & $3.82(\mathrm{dd}, 11.5,7.5)$ & 0.00 \\
\hline $11 b$ & $4.24(\mathrm{dd}, 11.5,4.5)$ & $4.24(\mathrm{dd}, 11.5,4.6)$ & 0.00 \\
\hline \multirow[t]{9}{*}{ Ac $x 9$} & $1.99(\mathrm{~s})$ & $1.99(\mathrm{~s})$ & 0.00 \\
\hline & $2.00(\mathrm{~s})$ & $2.00(\mathrm{~s})$ & 0.00 \\
\hline & $2.01(\mathrm{~s})$ & $2.01(\mathrm{~s})$ & 0.00 \\
\hline & $2.04(\mathrm{~s})$ & $2.06(\mathrm{~s})$ & -0.02 \\
\hline & $2.05(\mathrm{~s})$ & $2.06(\mathrm{~s})$ & -0.01 \\
\hline & $2.06(\mathrm{~s})$ & $2.07(\mathrm{~s})$ & -0.01 \\
\hline & $2.08(\mathrm{~s})$ & $2.08(\mathrm{~s})$ & 0.00 \\
\hline & $2.11(\mathrm{~s})$ & $2.11(\mathrm{~s})$ & 0.00 \\
\hline & $2.14(\mathrm{~s})$ & $2.14(\mathrm{~s})$ & 0.00 \\
\hline
\end{tabular}

${ }^{a}$ Internal standard was not indicated. 
Table S2. ${ }^{13} \mathrm{C}\left\{{ }^{1} \mathrm{H}\right\}$ NMR data of the reported ${ }^{\mathrm{S} 3}$ and synthetic diospyrodin nonaacetate (2) in $\mathrm{CDCl}_{3}$

\begin{tabular}{|c|c|c|c|}
\hline \multirow[b]{2}{*}{ No. } & reported $2(100 \mathrm{MHz})^{a}$ & \multirow{2}{*}{$\begin{array}{c}\text { synthetic } 2(100 \mathrm{MHz}) \\
{ }^{13} \mathrm{C}\left\{{ }^{1} \mathrm{H}\right\}[\delta]\end{array}$} & \multirow{2}{*}{$\Delta\left(\delta_{\text {reported }}-\delta_{\text {synthetic }}\right)$} \\
\hline & ${ }^{13} \mathrm{C}\left\{{ }^{1} \mathrm{H}\right\}[\delta]$ & & \\
\hline 1 & 61.80 & 61.75 & 0.05 \\
\hline 2 & 76.27 & 76.26 & 0.01 \\
\hline 3 & 67.81 & 67.74 & 0.07 \\
\hline 4 & 74.17 & 74.15 & 0.02 \\
\hline 5 & 67.34 & 67.30 & 0.04 \\
\hline 6 & 77.58 & 77.61 & -0.03 \\
\hline 7 & 67.91 & 67.84 & 0.07 \\
\hline 8 & 66.17 & 66.12 & 0.05 \\
\hline 9 & 67.75 & 67.71 & 0.04 \\
\hline 10 & 69.16 & 69.10 & 0.06 \\
\hline 11 & 62.16 & 62.17 & -0.01 \\
\hline \multirow[t]{18}{*}{ Ac $\times 9$} & 20.48 & 20.51 & -0.03 \\
\hline & 20.52 & 20.55 & -0.03 \\
\hline & 20.52 & 20.55 & -0.03 \\
\hline & 20.58 & 20.61 & -0.03 \\
\hline & 20.60 & 20.64 & -0.04 \\
\hline & 20.67 & 20.71 & -0.04 \\
\hline & 20.69 & 20.72 & -0.03 \\
\hline & 20.80 & 20.84 & -0.04 \\
\hline & 20.83 & 20.87 & -0.04 \\
\hline & 169.14 & 169.20 & -0.06 \\
\hline & 169.21 & 169.27 & -0.06 \\
\hline & 169.58 & 169.64 & -0.06 \\
\hline & 169.62 & 169.69 & -0.07 \\
\hline & 170.22 & 170.29 & -0.07 \\
\hline & 170.31 & 170.38 & -0.07 \\
\hline & 170.37 & 170.45 & -0.08 \\
\hline & 170.40 & 170.48 & -0.08 \\
\hline & 170.46 & 170.54 & -0.08 \\
\hline
\end{tabular}

${ }^{a}$ Internal standard was not indicated. 


\section{Comparison of ${ }^{1} \mathrm{H}$ and ${ }^{13} \mathrm{C}\left\{{ }^{1} \mathrm{H}\right\}$ NMR data of the reported and synthetic diospyrodin (1) in $\mathrm{D}_{2} \mathrm{O}$}

Table S3. ${ }^{1} \mathrm{H}$ NMR data of the reported ${ }^{\mathrm{S} 3}$ and synthetic diospyrodin (1) in $\mathrm{D}_{2} \mathrm{O}$

\begin{tabular}{rlll}
\hline & ${\text { reported } \mathbf{1}(400 \mathrm{MHz})^{a}}$ & synthetic $\mathbf{1}(400 \mathrm{MHz})^{b}$ & $\Delta\left(\delta_{\text {reported }}-\delta_{\text {synthetic }}\right)$ \\
\hline No. & ${ }^{1} \mathrm{H}[\delta$, multi, $J(\mathrm{~Hz})]$ & ${ }^{1} \mathrm{H}[\delta$, multi, $J(\mathrm{~Hz})]$ & \\
\hline $1 \mathrm{a}$ & $3.66(\mathrm{dd}, 12.0,2.0)$ & $3.72(\mathrm{~m})$ & -0.06 \\
\hline $\mathrm{b}$ & $3.91(\mathrm{dd}, 12.0,4.5)$ & $3.89(\mathrm{~d}, 12.4)$ & 0.02 \\
3 & $3.36(\mathrm{ddd}, 9.5,4.5,2.0)$ & $3.38(\mathrm{~m})$ & -0.02 \\
4 & $3.37(\mathrm{dd}, 9.5,9.5)$ & $3.55(\mathrm{~m})$ & -0.18 \\
4 & $3.48(\mathrm{dd}, 9.5,9.5)$ & $3.54(\mathrm{~m})$ & -0.06 \\
5 & $3.68(\mathrm{dd}, 9.5,9.5)$ & $3.66(\mathrm{~m})$ & 0.02 \\
6 & $3.35(\mathrm{dd}, 9.5,4.5)$ & $3.38(\mathrm{~m})$ & -0.03 \\
7 & $3.51(\mathrm{dd}, 4.5,1.5)$ & $3.54(\mathrm{~m})$ & -0.03 \\
8 & $4.13(\mathrm{dd}, 4.5,1.5)$ & $4.17(\mathrm{~d}, 3.2)$ & -0.04 \\
9 & $3.89(\mathrm{dd}, 9.5,1.5)$ & $3.92(\mathrm{~d}, 9.6)$ & -0.03 \\
10 & $3.93(\mathrm{ddd}, 9.5,4.5,1.5)$ & $3.97(\mathrm{dd}, 7.3,7.3)$ & -0.04 \\
$11 \mathrm{a}$ & $3.61(\mathrm{dd}, 11.0,6.5)$ & $3.67(\mathrm{~m})$ & -0.06 \\
$11 \mathrm{~b}$ & $3.64(\mathrm{dd}, 11.0,4.5)$ & $3.69(\mathrm{~m})$ & -0.05 \\
\hline
\end{tabular}

${ }^{a}$ Internal standard was not indicated. ${ }^{b}$ Chemical shifts were assigned by using ${ }^{1} \mathrm{H}_{-}{ }^{13} \mathrm{C}$ HMQC spectra.

Table S4. ${ }^{13} \mathrm{C}\left\{{ }^{1} \mathrm{H}\right\}$ NMR data of the reported ${ }^{\mathrm{S} 3}$ and synthetic diospyrodin (1) in $\mathrm{D}_{2} \mathrm{O}$

\begin{tabular}{rccc}
\hline & reported $\mathbf{1}(100 \mathrm{MHz})^{a}$ & synthetic $\mathbf{1}(100 \mathrm{MHz})$ & $\Delta\left(\delta_{\text {reported }}-\delta_{\text {synthetic }}\right)$ \\
\cline { 2 - 4 } No. & ${ }^{13} \mathrm{C}\left\{{ }^{1} \mathrm{H}\right\}(\delta)$ & ${ }^{13} \mathrm{C}\left\{{ }^{1} \mathrm{H}\right\}(\delta)$ & \\
\hline 1 & 66.24 & 61.51 & 4.73 \\
2 & 82.67 & 77.95 & 4.72 \\
3 & 74.83 & 70.10 & 4.73 \\
4 & 84.90 & 80.23 & 4.67 \\
5 & 74.23 & 69.48 & 4.75 \\
6 & 84.98 & 80.28 & 4.70 \\
7 & 76.81 & 72.09 & 4.72 \\
8 & 74.96 & 70.22 & 4.74 \\
9 & 73.82 & 69.10 & 4.72 \\
10 & 75.34 & 70.62 & 4.72 \\
11 & 68.45 & 63.74 & 4.71 \\
\hline
\end{tabular}

${ }^{a}$ Internal standard was not indicated. 


\section{Structural determination of 3- $\alpha$}

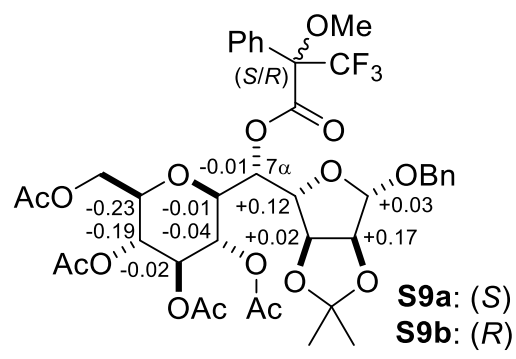

Figure S2. Determination of the C7-stereochemistry of alcohol 3- $\alpha$. The values are the differences $(\Delta \delta)$ in ${ }^{1} \mathrm{H}$ chemical shifts between $\mathbf{S 9 a}$ and $\mathbf{S 9 b}(\Delta \delta=\delta(\mathbf{S 9 a})-\delta(\mathbf{S 9 b}))$ in $\mathrm{CD}_{3} \mathrm{OD}$.

The C7-configuration of alcohol 3- $\alpha$ was determined by the application of the modified Mosher method (Figure S2). ${ }^{\text {S4 }}$ Namely, 3- $\alpha$ was derivatized into $(S)$-MTPA ester S9a and $(R)$-MTPA ester S9b. The difference in ${ }^{1} \mathrm{H}$ NMR chemical shifts between $\mathbf{S 9 a}$ and $\mathbf{S 9 b}$ was calculated to confirm the C7 $\alpha$-stereochemistry of $3-\alpha$.

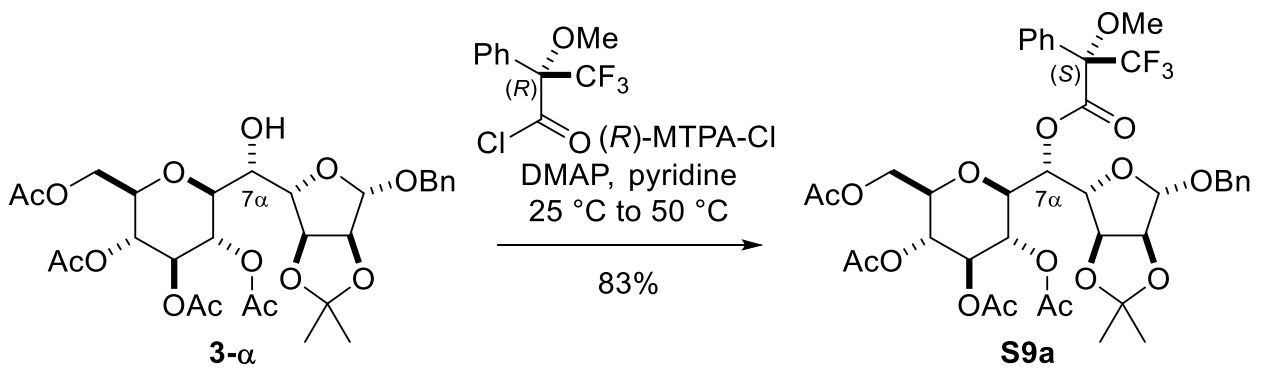

MTPA ester S9a. DMAP $(1.0 \quad \mathrm{mg}, \quad 8.2 \mu \mathrm{mol})$ and $(R)$ - $\alpha$-methoxy- $\alpha$ (trifluoromethyl)phenylacetyl chloride $((R)-\mathrm{MTPACl}, 15.6 \mu \mathrm{L}, 83.4 \mu \mathrm{mol})$ were successively added to a solution of alcohol 3- $\alpha(10.2 \mathrm{mg}, 16.7 \mu \mathrm{mol})$ in pyridine $(855 \mu \mathrm{L})$ at $0{ }^{\circ} \mathrm{C}$. After the reaction mixture was stirred at $25^{\circ} \mathrm{C}$ for $3.5 \mathrm{~h}$, additional $(R)-\mathrm{MTPACl}(31.2 \mu \mathrm{L}, 167 \mu \mathrm{mol})$ was added at $25{ }^{\circ} \mathrm{C}$. After being stirred at $50{ }^{\circ} \mathrm{C}$ for $21 \mathrm{~h}$, the reaction mixture was concentrated. The residue was filtered through a pad of amine-modified silica gel $(1 \mathrm{~g}$, EtOAc) and the filtrate was concentrated. The residue was purified by PTLC (hexane/EtOAc $=3 / 2)$ to afford MTPA ester S9a $(11.4 \mathrm{mg}, 13.8 \mu \mathrm{mol})$ in $83 \%$ yield: colorless oil; $[\alpha]_{\mathrm{D}}{ }^{26}+36.1$ (c $0.57, \mathrm{CHCl}_{3}$ ); IR (film) 2948, 1754, 1446, 1374, 1228, 1089, 1036, 870, $730 \mathrm{~cm}^{-1} ;{ }^{1} \mathrm{H}$ NMR (400 MHz, $\left.\mathrm{CD}_{3} \mathrm{OD}\right) \delta 1.24\left(3 \mathrm{H}, \mathrm{s}, \mathrm{CH}_{3}\right.$ of acetonide), $1.46\left(3 \mathrm{H}, \mathrm{s}, \mathrm{CH}_{3}\right.$ of acetonide), 1.59 (3H, s, $\left.\mathrm{CH}_{3} \mathrm{CO}\right), 1.95\left(3 \mathrm{H}, \mathrm{s}, \mathrm{CH}_{3} \mathrm{CO}\right), 2.00\left(3 \mathrm{H}, \mathrm{s}, \mathrm{CH}_{3} \mathrm{CO}\right), 2.02\left(3 \mathrm{H}, \mathrm{s}, \mathrm{CH}_{3} \mathrm{CO}\right), 3.63(3 \mathrm{H}, \mathrm{s}$, $\left.\mathrm{OCH}_{3}\right), 3.83(1 \mathrm{H}, \mathrm{ddd}, J=10.1,3.2,3.2 \mathrm{~Hz}, \mathrm{H} 2), 4.06(1 \mathrm{H}, \mathrm{d}, J=10.5 \mathrm{~Hz}, \mathrm{H} 6), 4.17$ (2H, d, $J$ 
$=3.2 \mathrm{~Hz}, \mathrm{H1}), 4.44(1 \mathrm{H}, \mathrm{d}, J=10.5 \mathrm{~Hz}, \mathrm{H} 8), 4.52(1 \mathrm{H}, \mathrm{d}, J=6.0 \mathrm{~Hz}, \mathrm{H} 10), 4.64(1 \mathrm{H}, \mathrm{d}, J=$ $12.4 \mathrm{~Hz}, \mathrm{OCHaHbPh}), 4.66(1 \mathrm{H}, \mathrm{dd}, J=10.5,8.7 \mathrm{~Hz}, \mathrm{H} 5), 4.71(1 \mathrm{H}, \mathrm{d}, J=6.0 \mathrm{~Hz}, \mathrm{H} 9), 4.79$ $(1 \mathrm{H}, \mathrm{dd}, J=10.1,9.6 \mathrm{~Hz}, \mathrm{H} 3), 4.81(1 \mathrm{H}, \mathrm{d}, J=12.4 \mathrm{~Hz}, \mathrm{OCHa} H \mathrm{bPh}), 5.22(1 \mathrm{H}, \mathrm{dd}, J=9.6$, $8.7 \mathrm{~Hz}, \mathrm{H} 4), 5.22$ (1H, s, H11), 5.23 (1H, d, $J=10.5 \mathrm{~Hz}, \mathrm{H} 7), 7.30$ (1H, m, aromatic), 7.36$7.47\left(7 \mathrm{H}, \mathrm{m}\right.$, aromatic), 7.66-7.68 (2H, m, aromatic); ${ }^{13} \mathrm{C}\left\{{ }^{1} \mathrm{H}\right\} \mathrm{NMR}\left(100 \mathrm{MHz}, \mathrm{CD}_{3} \mathrm{OD}\right), \delta$ 20.3, 20.5, 20.6 (2C), 24.9, 26.7, 56.5, 62.8, 69.1, 69.2, 71.8, 72.3, 75.5, 75.9, 76.7, 82.9, 84.7, 85.7 (q, $J=27.3 \mathrm{~Hz}), 86.4,109.9,113.9,124.7$ (q, $J=289.3 \mathrm{~Hz}), 128.7$ (2C), $128.9(2 \mathrm{C}), 129.0$, 129.6 (2C), 129.7 (2C), 131.0, 133.2, 138.7, 167.2, 171.0, 171.1, 171.8, 172.3; HRMS (ESITOF) $[\mathrm{M}+\mathrm{Na}]^{+} \mathrm{m} / \mathrm{z}$ : Calcd for $\mathrm{C}_{39} \mathrm{H}_{45} \mathrm{~F}_{3} \mathrm{O}_{16} \mathrm{Na} \quad 849.2552$; Found 849.2536.
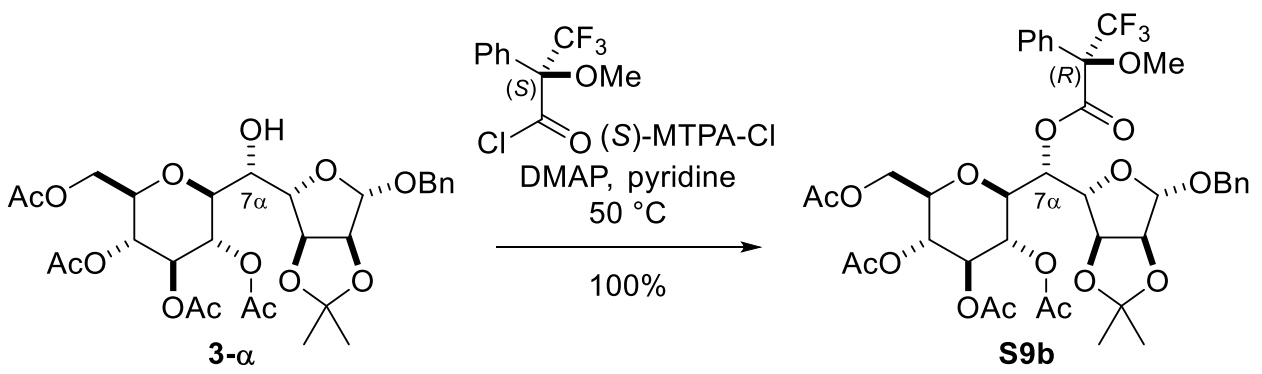

MTPA ester S9b. DMAP $(1.0 \mathrm{mg}, 8.2 \mu \mathrm{mol})$ and (S)-MTPACl $(61.8 \mu \mathrm{L}, 330 \mu \mathrm{mol})$ were successively added to a solution of alcohol 3- $\alpha(10.1 \mathrm{mg}, 16.5 \mu \mathrm{mol})$ in pyridine $(825 \mu \mathrm{L})$ at $0{ }^{\circ} \mathrm{C}$. After being stirred at $50^{\circ} \mathrm{C}$ for $4 \mathrm{~h}$, the reaction mixture was concentrated. The residue was filtered through a pad of amine-modified silica gel ( $1 \mathrm{~g}$, EtOAc) and the filtrate was concentrated. The residue was purified by PTLC (hexane/EtOAc $=3 / 2$ ) to afford MTPA ester S9b $(13.7 \mathrm{mg}, 16.5 \mu \mathrm{mol})$ in $100 \%$ yield: colorless oil; $[\alpha]_{\mathrm{D}}{ }^{27}+33.0\left(c 0.42, \mathrm{CHCl}_{3}\right)$; IR (film) 2941, 1757, 1454, 1374, 1230, 1082, 1038, $869 \mathrm{~cm}^{-1}$; ${ }^{1} \mathrm{H}$ NMR (400 MHz, CD $\left.{ }_{3} \mathrm{OD}\right) \delta 1.20(3 \mathrm{H}$, s, $\mathrm{CH}_{3}$ of acetonide), $1.37\left(3 \mathrm{H}, \mathrm{s}, \mathrm{CH}_{3}\right.$ of acetonide), $1.60\left(3 \mathrm{H}, \mathrm{s}, \mathrm{CH}_{3} \mathrm{CO}\right), 1.92\left(3 \mathrm{H}, \mathrm{s}, \mathrm{CH}_{3} \mathrm{CO}\right)$, $2.04\left(6 \mathrm{H}, \mathrm{s}, \mathrm{CH}_{3} \mathrm{CO} \times 2\right), 3.63\left(3 \mathrm{H}, \mathrm{s}, \mathrm{OCH}_{3}\right), 3.88(1 \mathrm{H}, \mathrm{ddd}, J=10.0,4.6,2.3 \mathrm{~Hz}, \mathrm{H} 2), 4.07$ $(1 \mathrm{H}, \mathrm{d}, J=10.5 \mathrm{~Hz}, \mathrm{H} 6), 4.17(1 \mathrm{H}, \mathrm{dd}, J=12.8,2.3 \mathrm{~Hz}, \mathrm{H} 1 \mathrm{a}), 4.27(1 \mathrm{H}, \mathrm{dd}, J=12.8,4.6 \mathrm{~Hz}$, H1b), 4.33 (1H, d, $J=10.5 \mathrm{~Hz}, \mathrm{H} 8), 4.35(1 \mathrm{H}, \mathrm{d}, J=6.0 \mathrm{~Hz}, \mathrm{H} 10), 4.64(1 \mathrm{H}, \mathrm{d}, J=11.9 \mathrm{~Hz}$, OCHaHbPh), 4.69 (1H, d, $J=6.0 \mathrm{~Hz}, \mathrm{H} 9), 4.70$ (1H, dd, $J=10.5,9.2 \mathrm{~Hz}, \mathrm{H} 5), 4.81$ (1H, d, $J$ $=11.9 \mathrm{~Hz}, \mathrm{OCHa} H \mathrm{bPh}), 4.98(1 \mathrm{H}, \mathrm{dd}, J=10.0,9.2 \mathrm{~Hz}, \mathrm{H} 3), 5.19(1 \mathrm{H}, \mathrm{s}, \mathrm{H} 11), 5.24(1 \mathrm{H}, \mathrm{dd}$, $J=10.0,9.2 \mathrm{~Hz}, \mathrm{H} 4), 5.24$ (1H, d, $J=10.6 \mathrm{~Hz}, \mathrm{H} 7), 7.29-7.53$ (8H, m, aromatic), 7.69-7.71 (2H, m, aromatic); ${ }^{13} \mathrm{C}\left\{{ }^{1} \mathrm{H}\right\} \mathrm{NMR}\left(100 \mathrm{MHz}, \mathrm{CD}_{3} \mathrm{OD}\right), \delta 20.3,20.5,20.6,20.7,24.9,26.6$, $56.3,62.9,69.1,69.2,71.9,72.2,75.7,76.0,76.9,82.8,84.4,86.3,110.1,113.7,128.88(2 \mathrm{C})$, 128.98, 129.04 (2C), 129.6 (2C), 129.8 (2C), 131.1, 132.5, 138.7, 167.0, 170.9, 171.2, 171.8, 
$172.3\left(\mathrm{CF}_{3}\right.$ and $\mathrm{PhCCF}_{3}(\mathrm{OMe})$ carbon peaks were not observed); HRMS (ESI-TOF) $[\mathrm{M}+\mathrm{Na}]^{+}$ $\mathrm{m} / \mathrm{z}$ : Calcd for $\mathrm{C}_{39} \mathrm{H}_{45} \mathrm{~F}_{3} \mathrm{O}_{16} \mathrm{Na}$ 849.2552; Found 849.2522.

\section{Structural determination of compounds $16,17, \mathrm{S7}$, and $\mathrm{S8}$ by ${ }^{13} \mathrm{C}\left\{{ }^{1} \mathrm{H}\right\} \mathrm{NMR}$ analysis} It was demonstrated that the ${ }^{13} \mathrm{C}\left\{{ }^{1} \mathrm{H}\right\}$ NMR chemical shifts of the two methyl groups of the 2,2'-dimethyl dioxolane ring are 25-27 ppm and 27-28 ppm in the cis-configuration, and are 27 ppm and $27 \mathrm{ppm}$ in the trans-counterpart. ${ }^{\mathrm{S} 5}$ Accordingly, the stereostructures of 16, 17, S7, and S8 were determined by the ${ }^{13} \mathrm{C}\left\{{ }^{1} \mathrm{H}\right\}$ NMR chemical shifts of the two methyl groups as shown below.
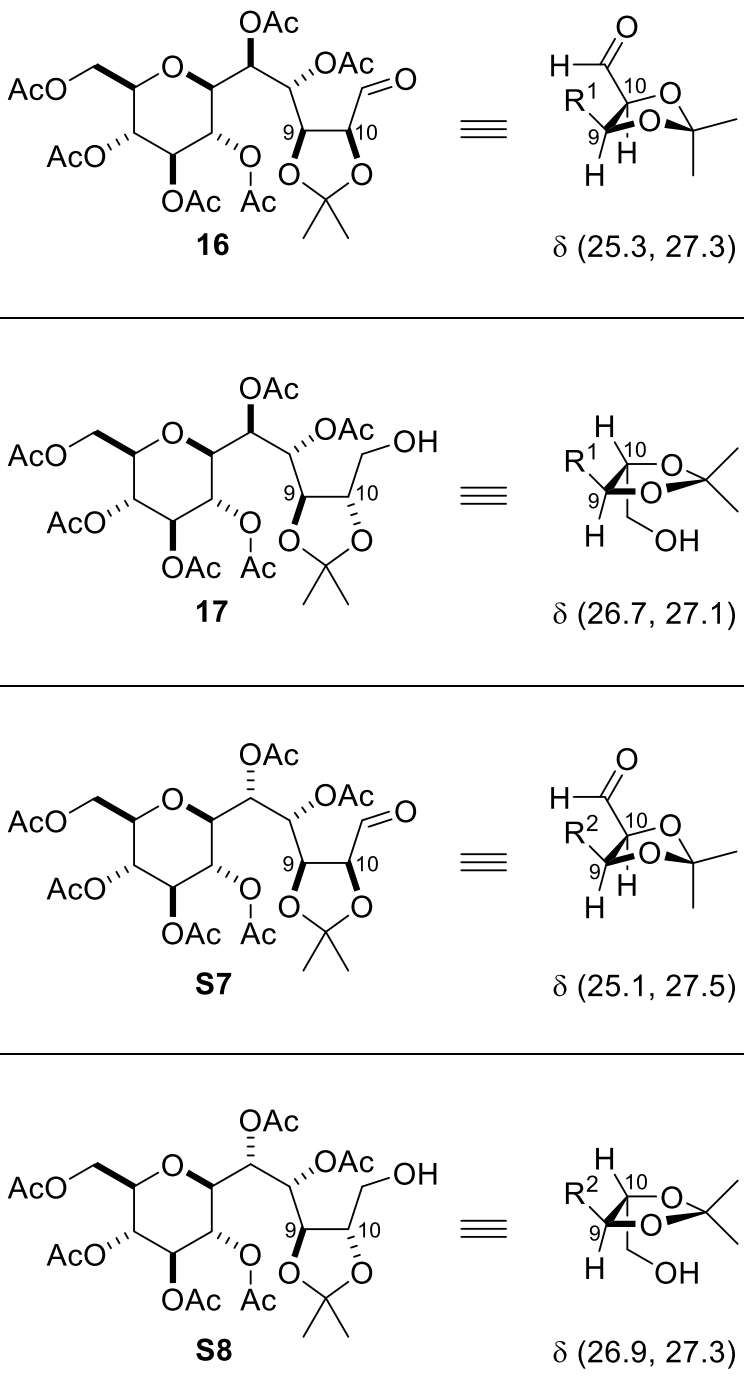


\section{References}

S1. Shulman, M. L.; Shiyan, S. D.; Khorlin A. Y. Carbohydr. Res. 1974, 33, 229-235.

S2. Norsikian, S.; Zeitouni, J.; Rat, S.; Gérard, S.; Lubineau, A. Carbohydr. Res. 2007, 342, 2716-2728.

S3. Dinda, B.; Bhattacharya, A.; De, U. C.; Arima, S.; Takayanagi, H.; Harigaya, Y. Chem. Pharm. Bull. 2006, 54, 679-681.

S4. Ohtani, I.; Kusumi, T.; Kashman, Y.; Kakisawa, H. J. Am. Chem. Soc. 1991, 113, 40924096.

S5. Dana, G.; Danechpajouh, H. Bull. Soc. Chim. Fr. II 1980, 395-399. 

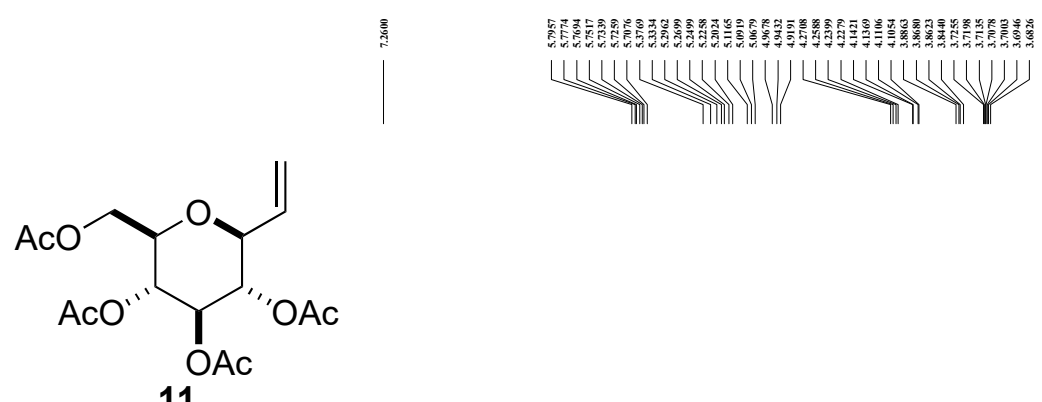

${ }^{1} \mathrm{H}$ NMR $\left(400 \mathrm{MHz}, \mathrm{CDCl}_{3}\right)$

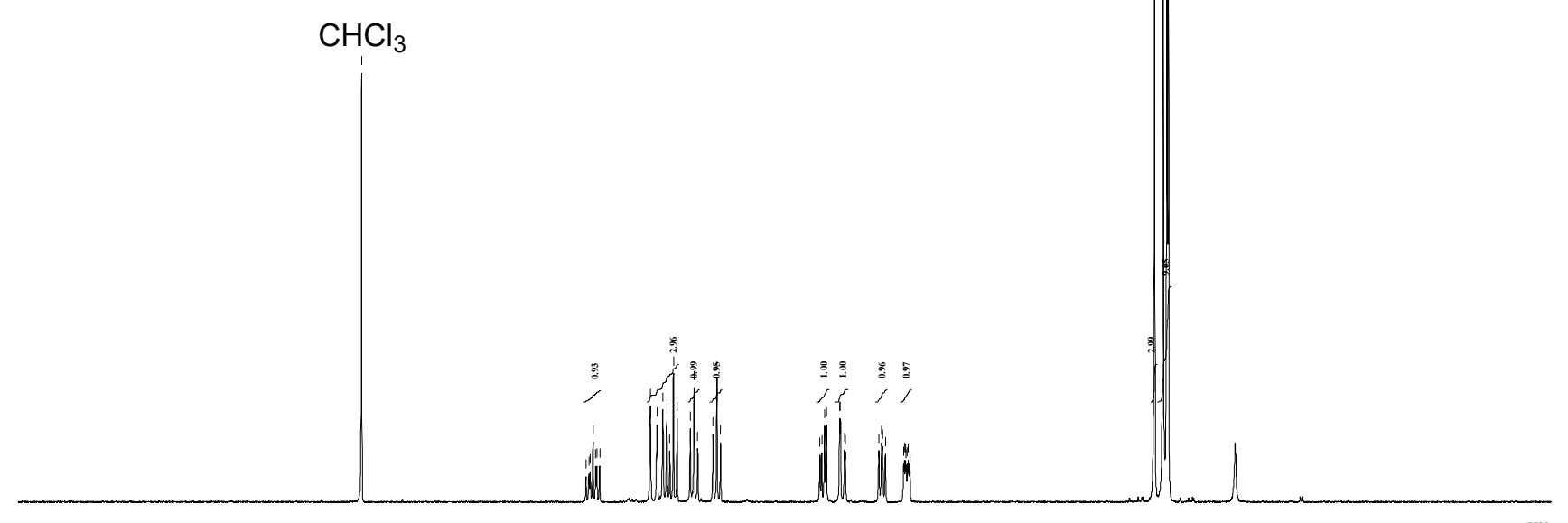

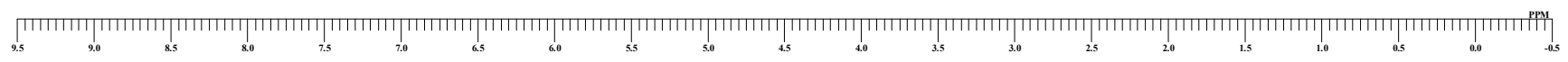

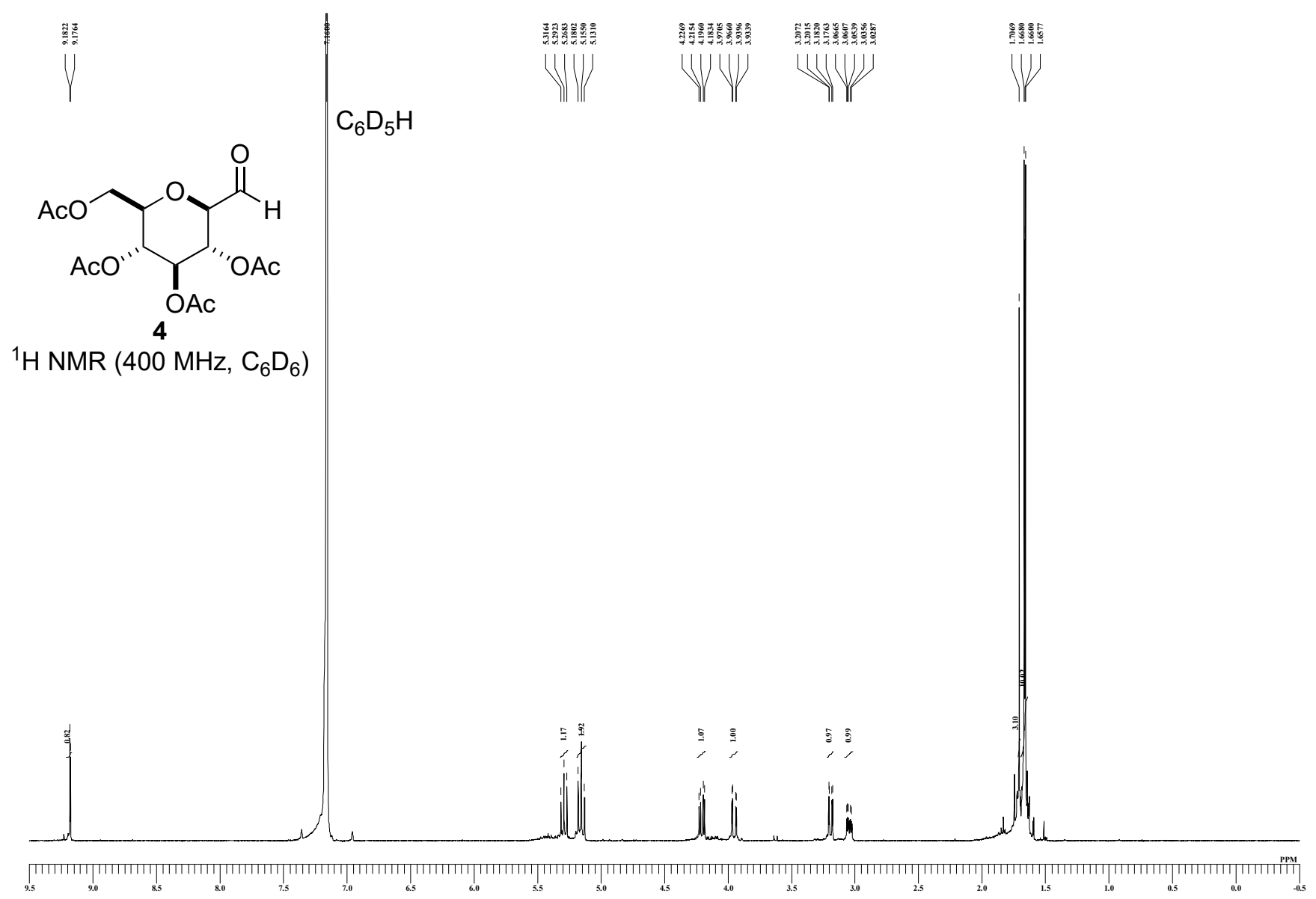


<smiles>CC1(C)O[C@@H]2[C@@H](CO)O[C@@H](OCc3ccccc3)[C@@H]2O1</smiles>

12

${ }^{1} \mathrm{H}$ NMR $\left(400 \mathrm{MHz}, \mathrm{CDCl}_{3}\right)$

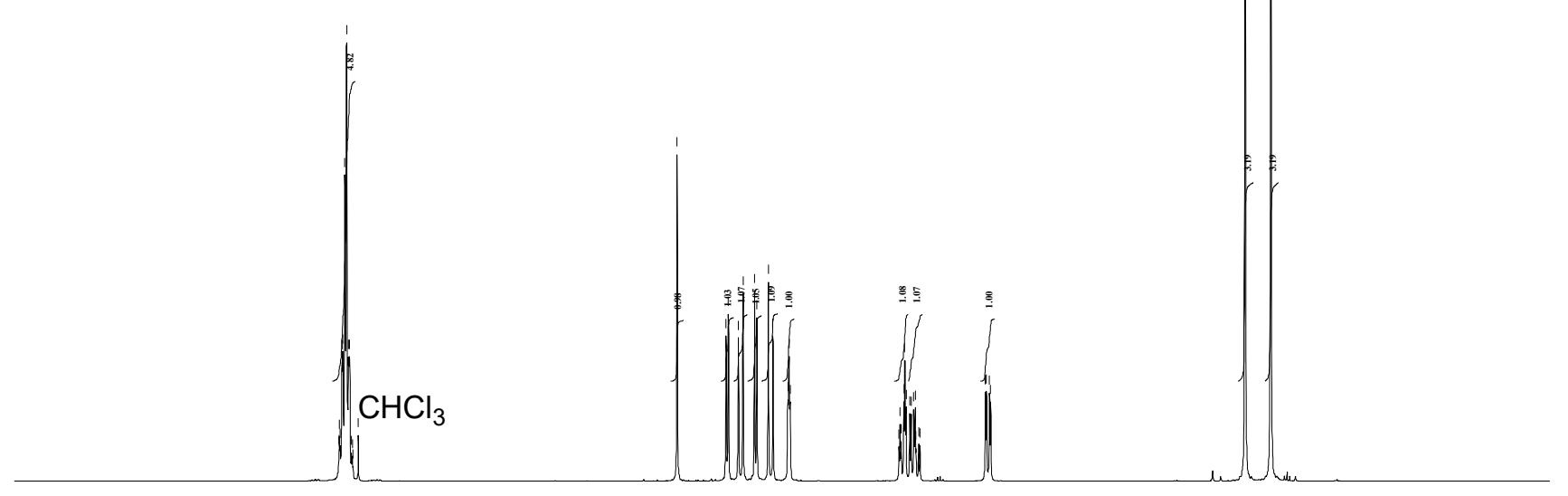

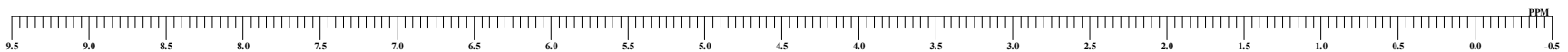

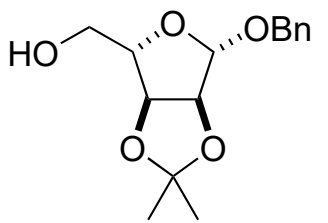

12

${ }^{13} \mathrm{C}$ NMR $\left(100 \mathrm{MHz}, \mathrm{CDCl}_{3}\right)$

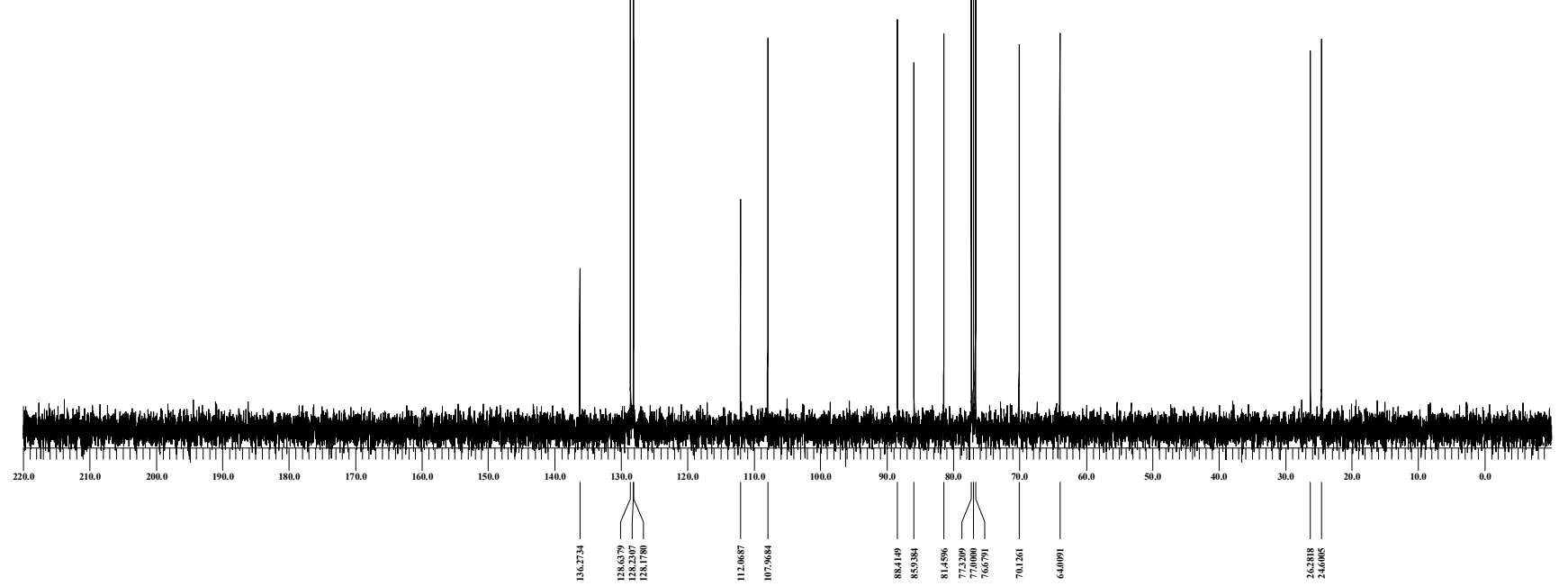


<smiles>CC1(C)OC2[C@H](O1)[C@@H](C(=O)O)O[C@H]2OCc1ccccc1</smiles>

13

${ }^{1} \mathrm{H}$ NMR $\left(400 \mathrm{MHz}, \mathrm{CDCl}_{3}\right)$

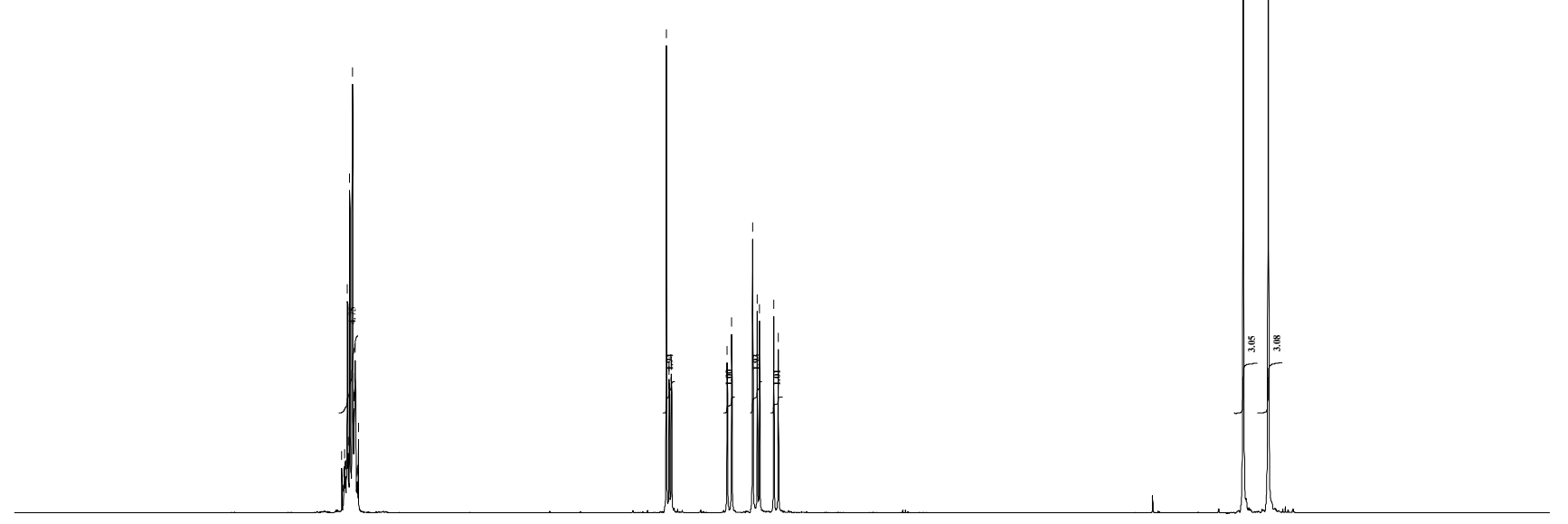

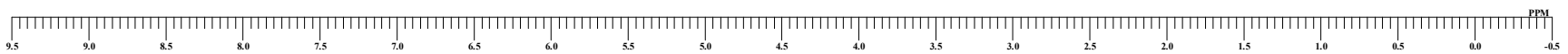<smiles>CC1(C)OC2[C@@H](O[C@H]2OCc2ccccc2)C1C(=O)O</smiles>

13

${ }^{13} \mathrm{C}$ NMR (100 MHz, $\left.\mathrm{CDCl}_{3}\right)$

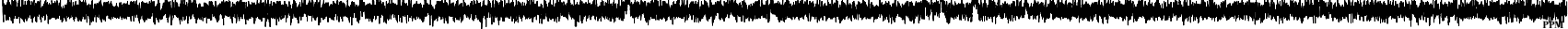
(

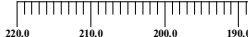


$\mathrm{PhTe}$<smiles>CC(=O)[C@H]1O[C@H](Cc2ccccc2)C2OC(C)(C)OC21</smiles>

5

${ }^{1} \mathrm{H}$ NMR $\left(400 \mathrm{MHz}, \mathrm{CDCl}_{3}\right)$

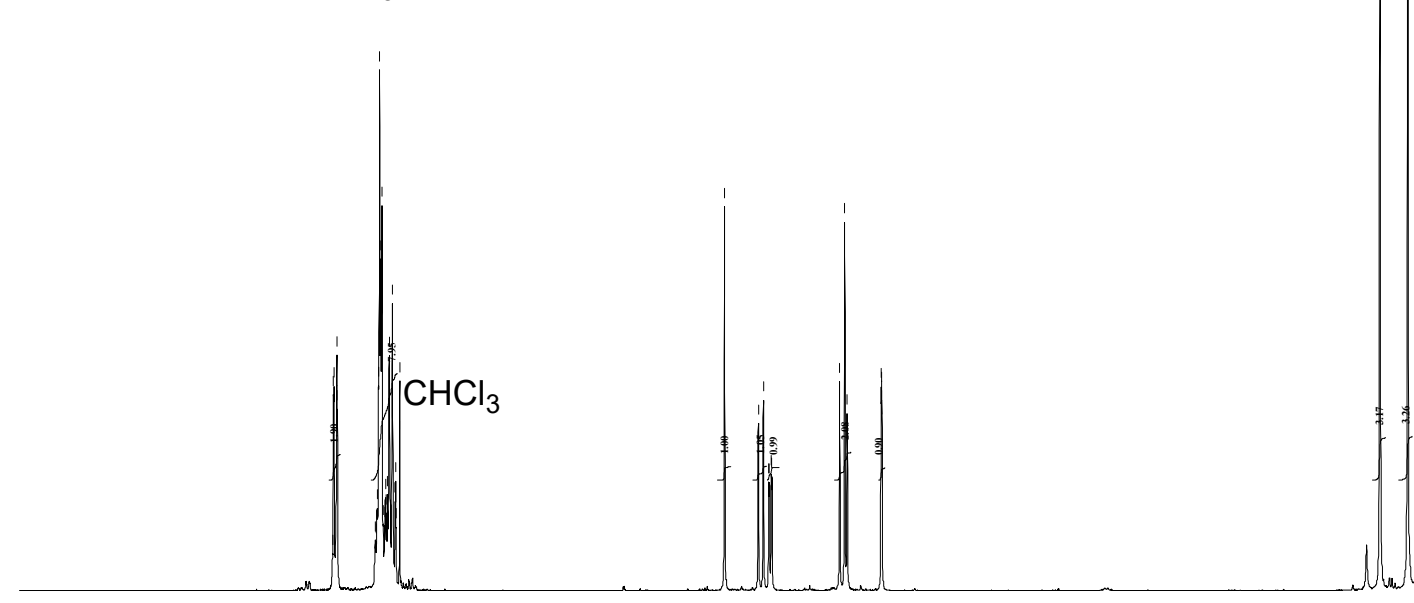

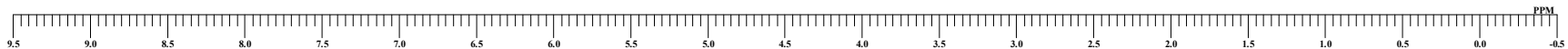<smiles>CC1(C)OC2C(C(=O)Oc3ccccc3)O[C@@H](Cc3ccccc3)[C@H]2O1</smiles>

$\mathrm{CDCl}_{3}$

5

${ }^{13} \mathrm{C} \mathrm{NMR}\left(100 \mathrm{MHz}, \mathrm{CDCl}_{3}\right)$

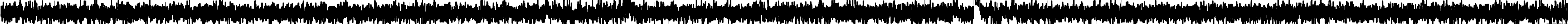

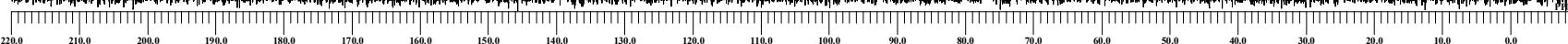

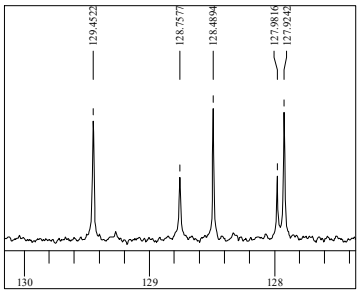
| 


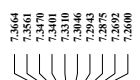

YII

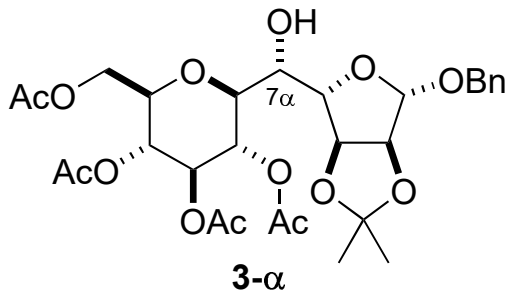

${ }^{1} \mathrm{H}$ NMR $\left(400 \mathrm{MHz}, \mathrm{CDCl}_{3}\right)$
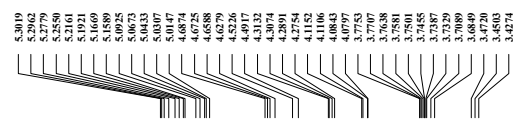
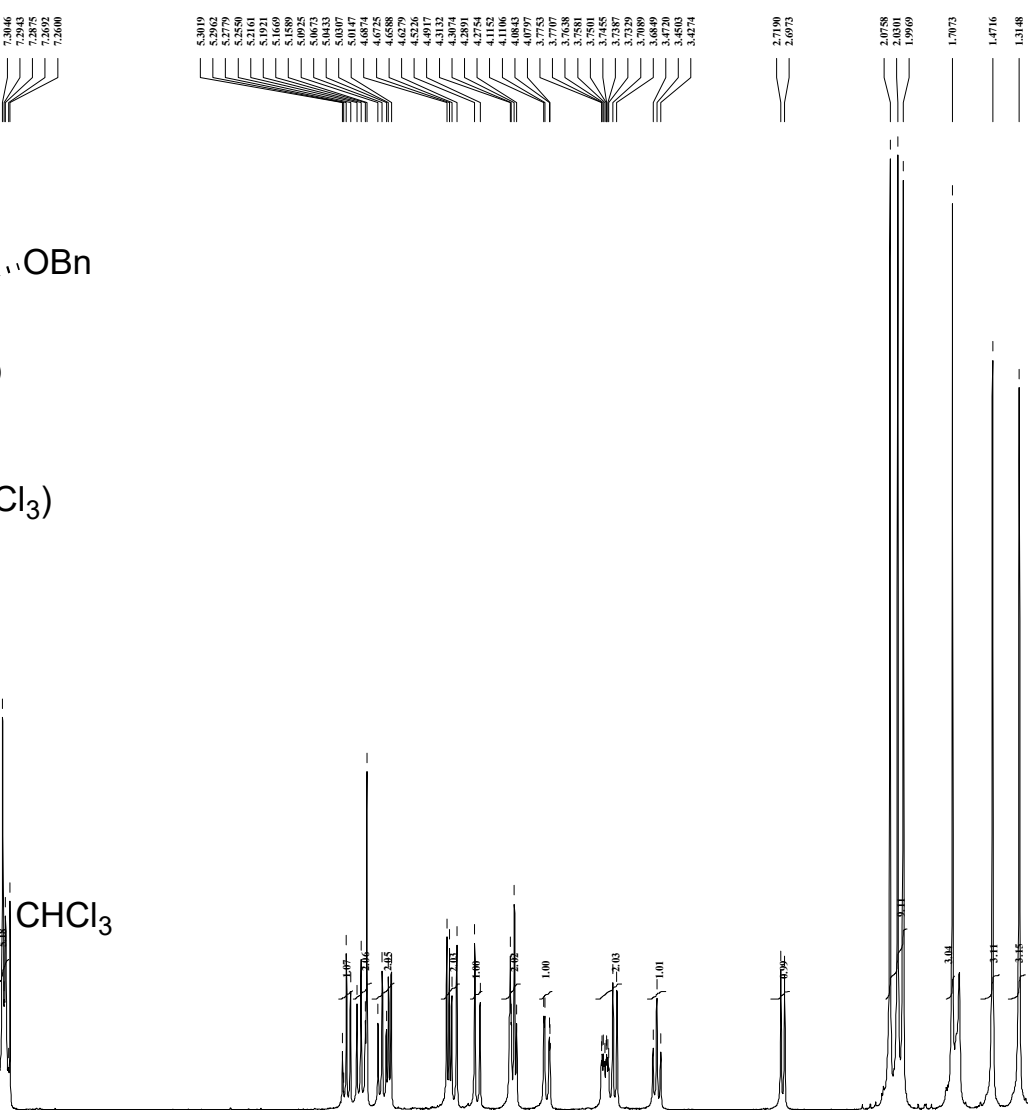

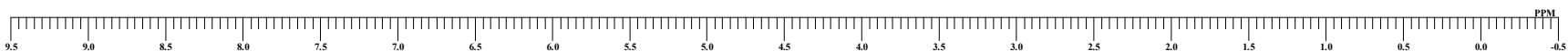<smiles>CC(=O)OC[C@H]1O[C@H]([C@H](O)[C@H]2O[C@H](Cc3ccccc3)C3OC(C)(C)OC32)[C@H](OC(C)=O)C(OC(C)=O)[C@H]1OC(C)=O</smiles>

3- $\alpha$

${ }^{13} \mathrm{C}$ NMR $\left(100 \mathrm{MHz}, \mathrm{CDCl}_{3}\right)$

$\mathrm{CDCl}_{3}$
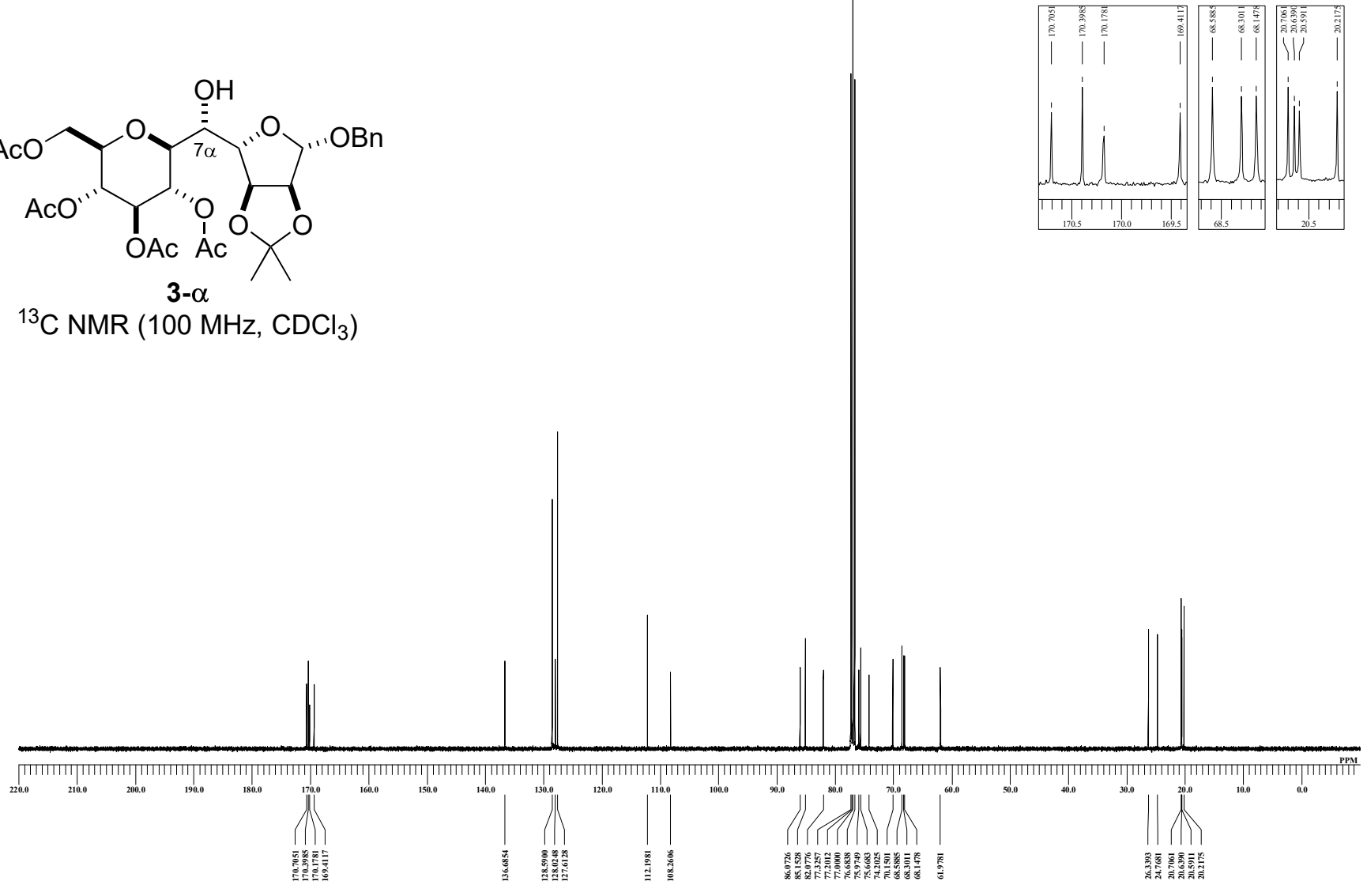

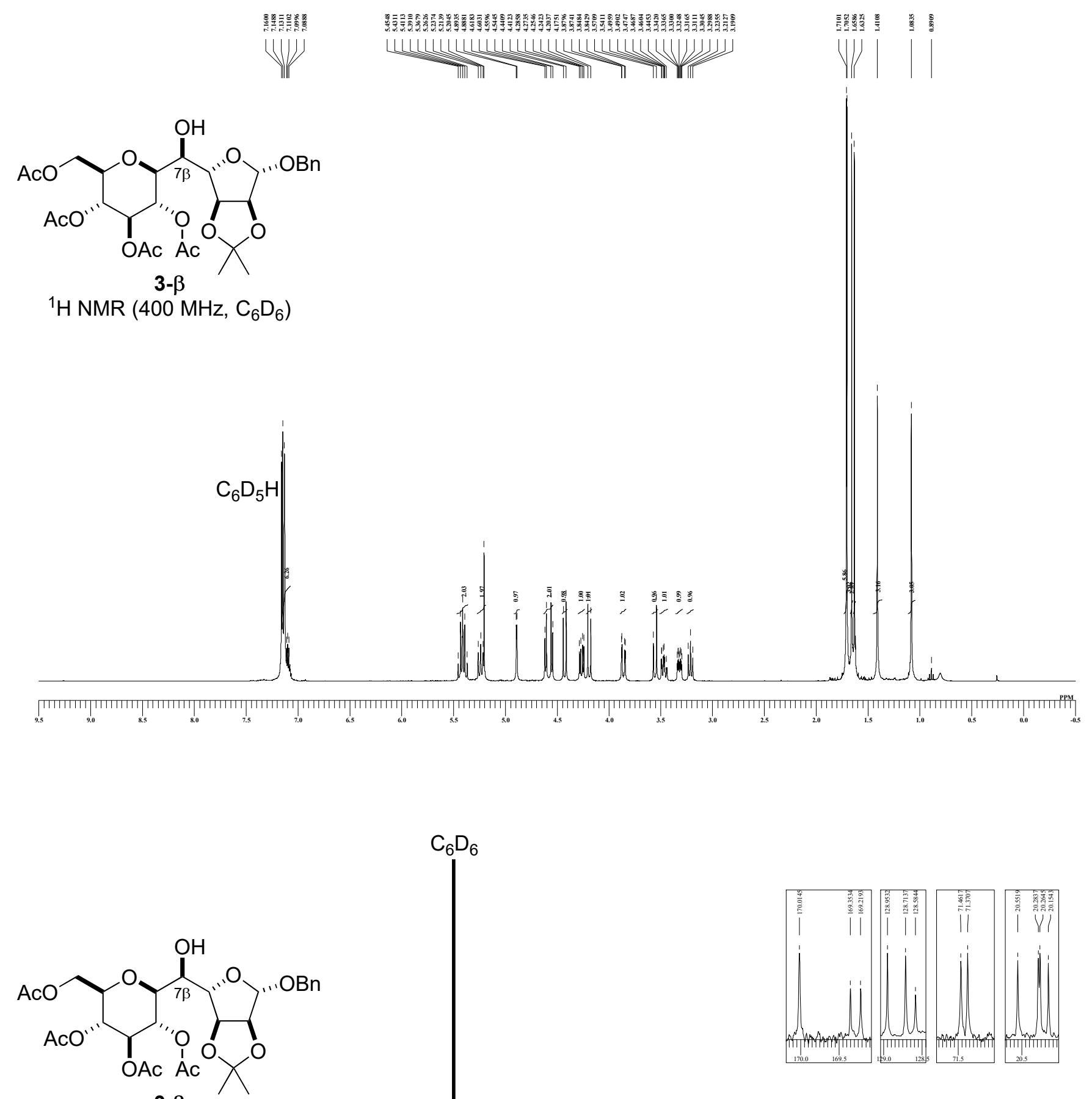

${ }^{13} \mathrm{C}$ NMR $\left(100 \mathrm{MHz}, \mathrm{C}_{6} \mathrm{D}_{6}\right)$

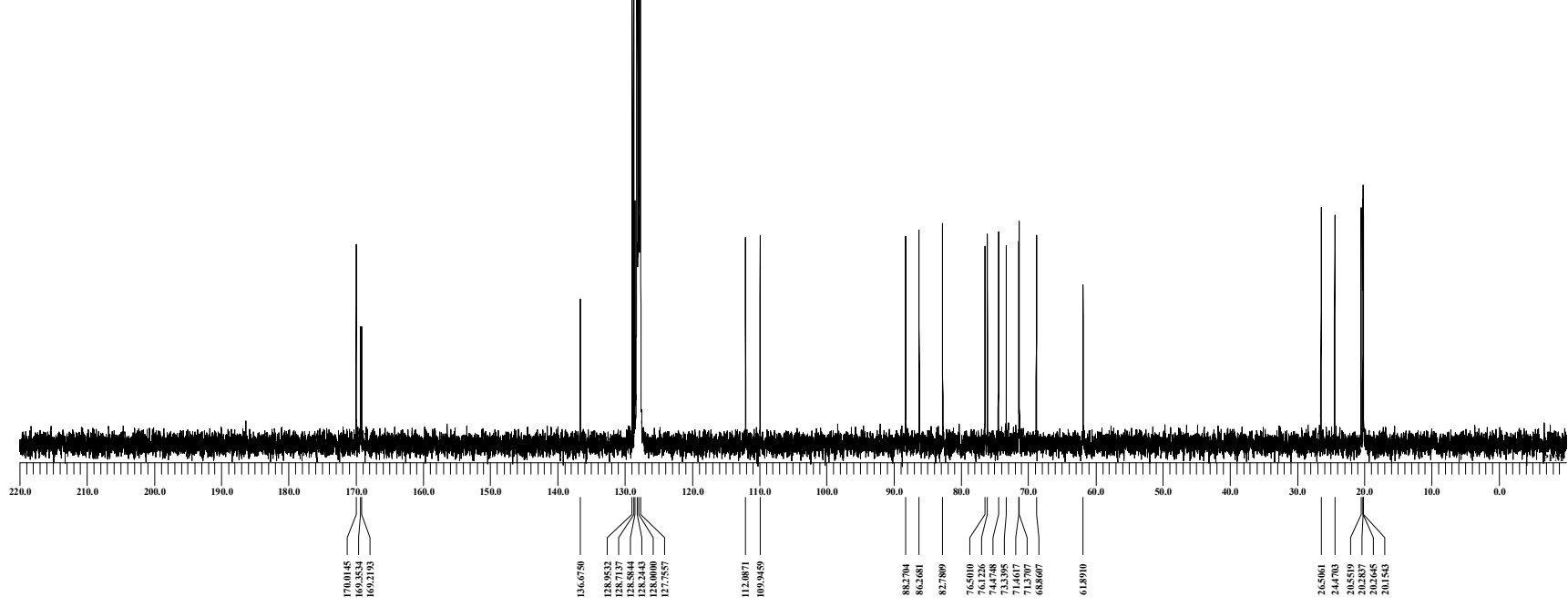




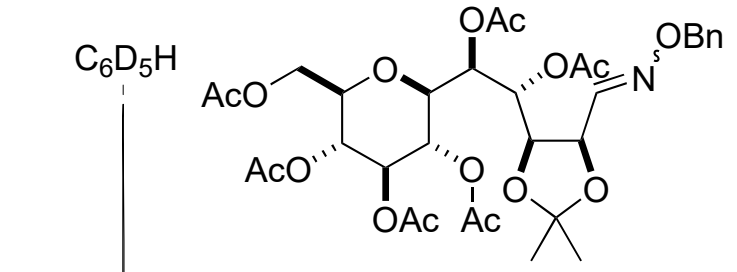

$15(E / Z=1: 0.2)$

${ }^{1} \mathrm{H}$ NMR $\left(400 \mathrm{MHz}, \mathrm{C}_{6} \mathrm{D}_{6}\right)$
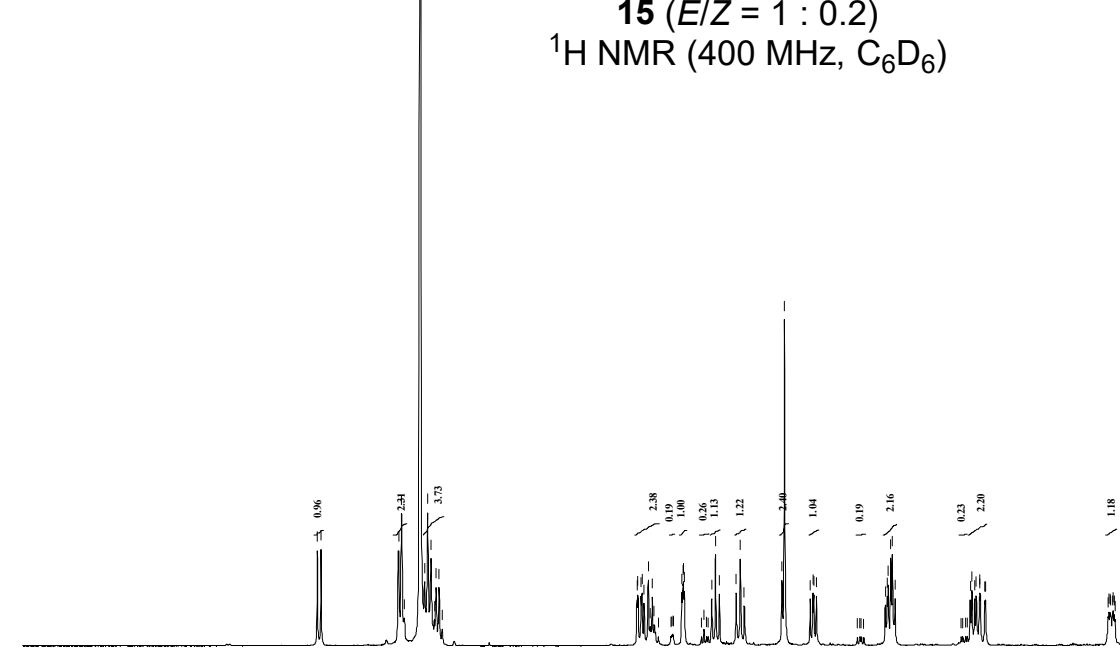

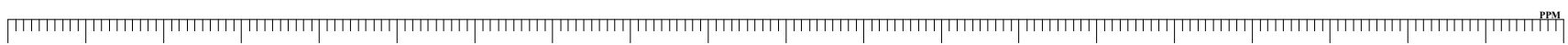

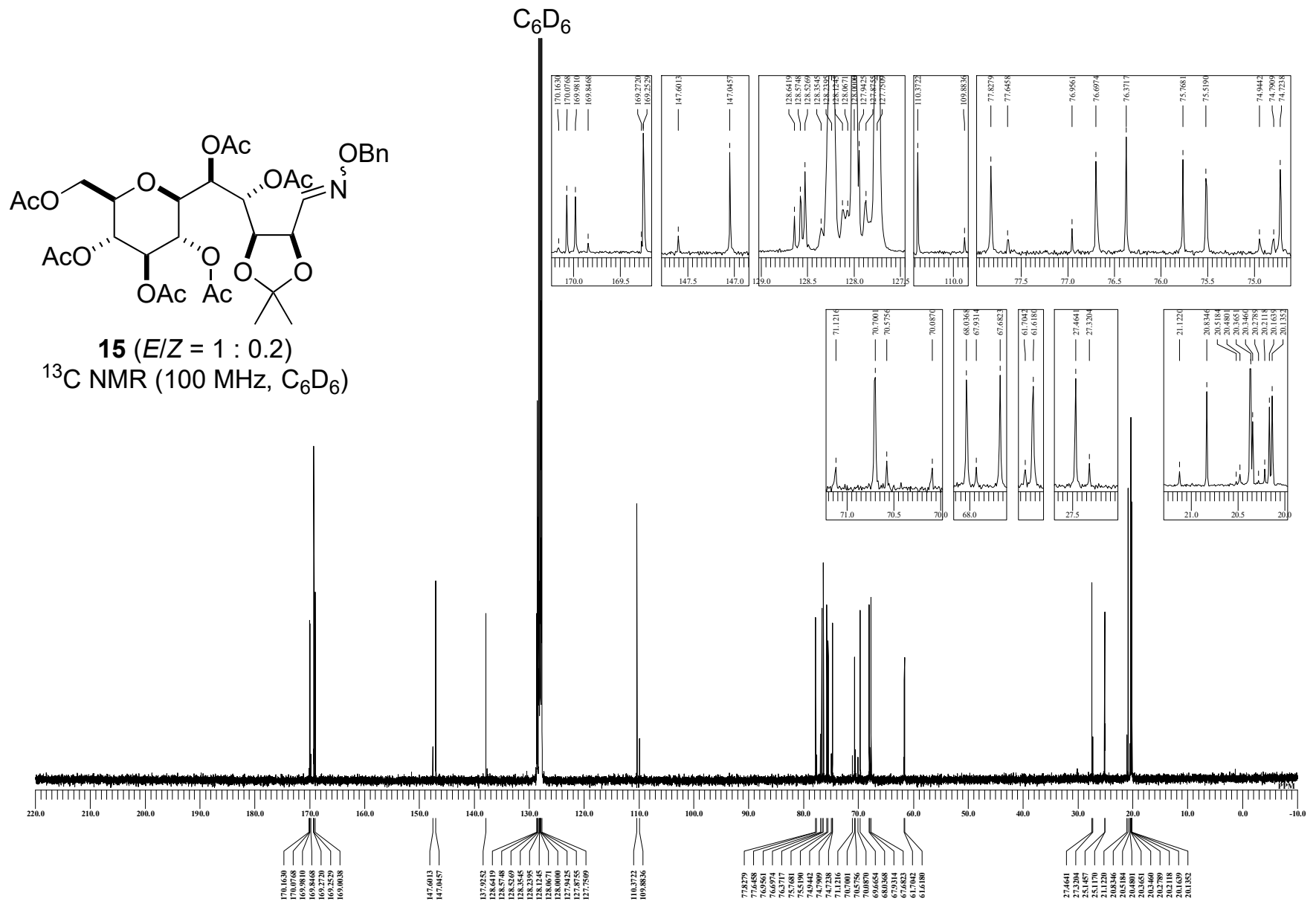



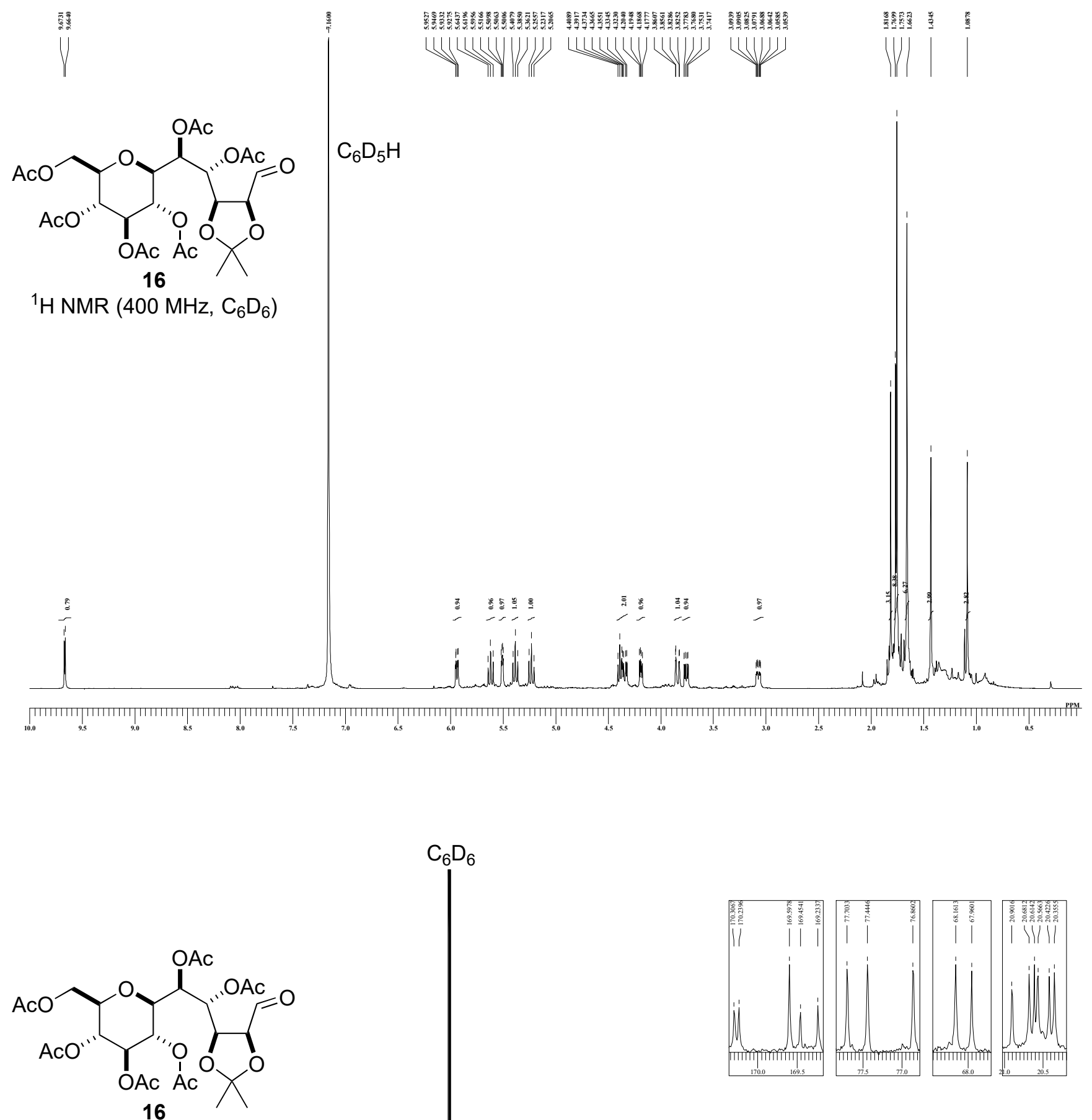

${ }^{13} \mathrm{C}$ NMR $\left(100 \mathrm{MHz}, \mathrm{C}_{6} \mathrm{D}_{6}\right)$

$\mathrm{C}_{6} \mathrm{D}_{6}$

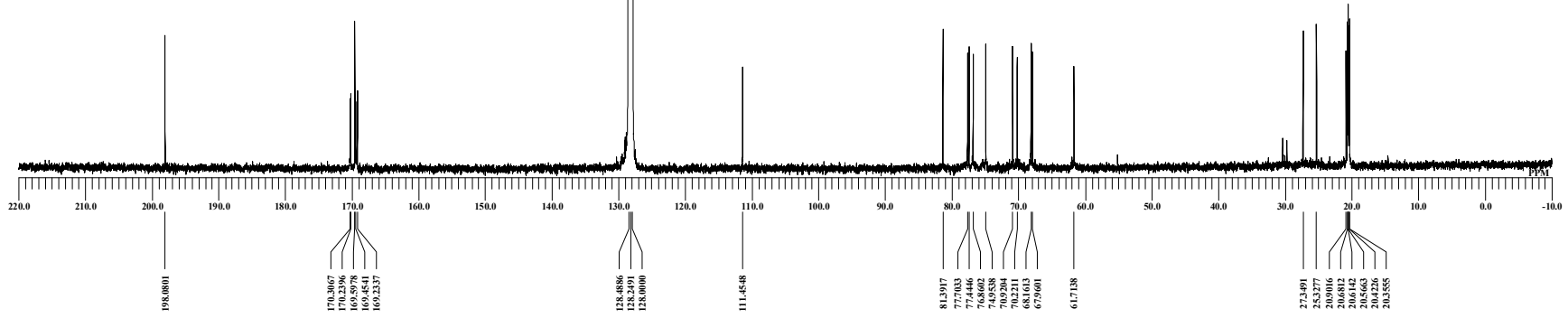



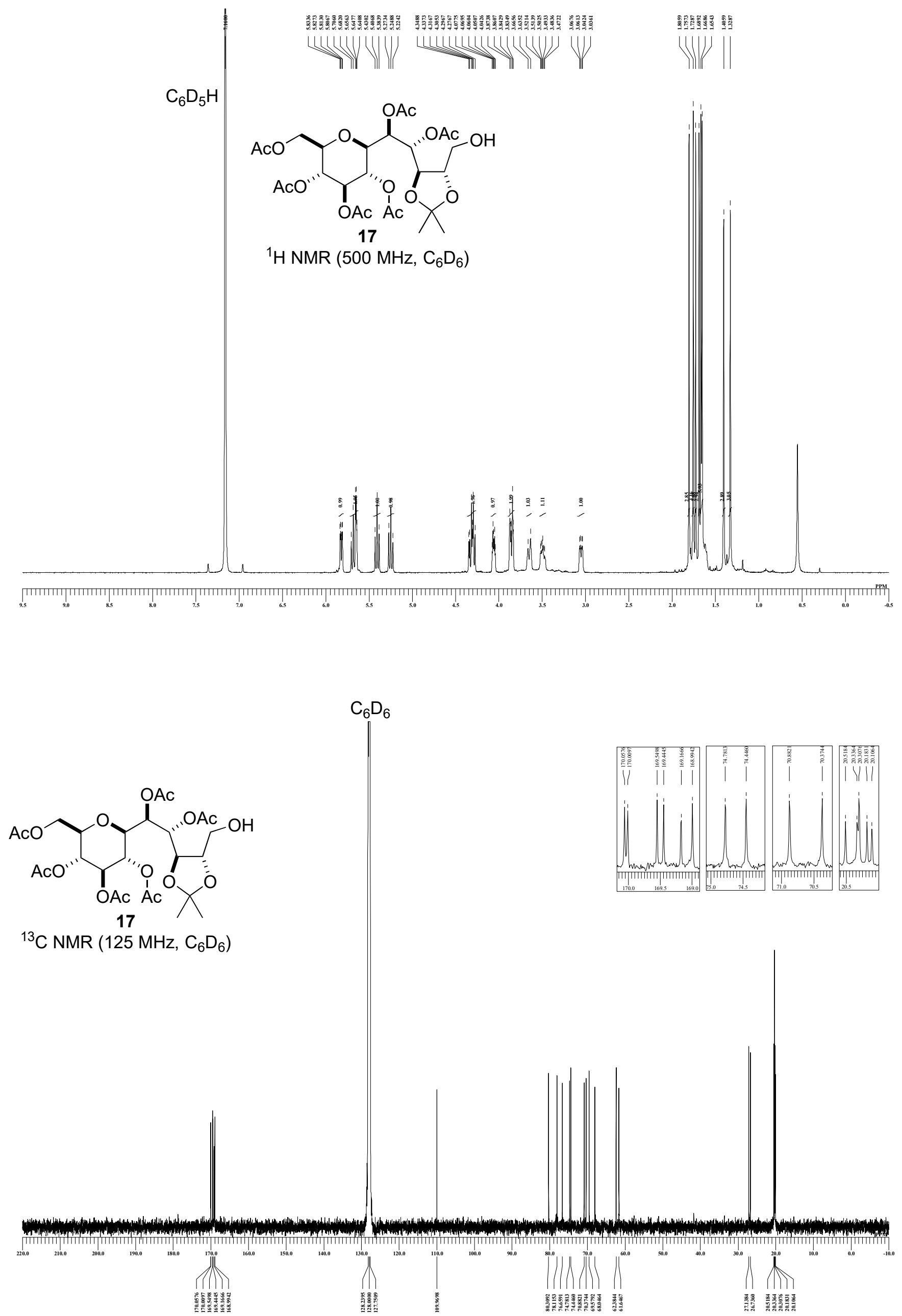


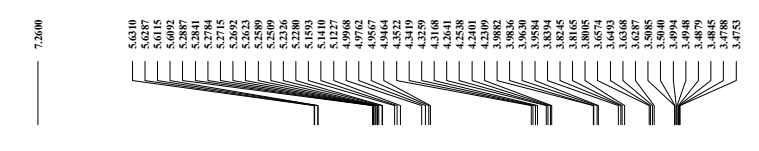

$\underbrace{O A C}_{O A c}$

diospyrodin nonaacetate (2)

${ }^{1} \mathrm{H}$ NMR $\left(500 \mathrm{MHz}, \mathrm{CDCl}_{3}\right)$

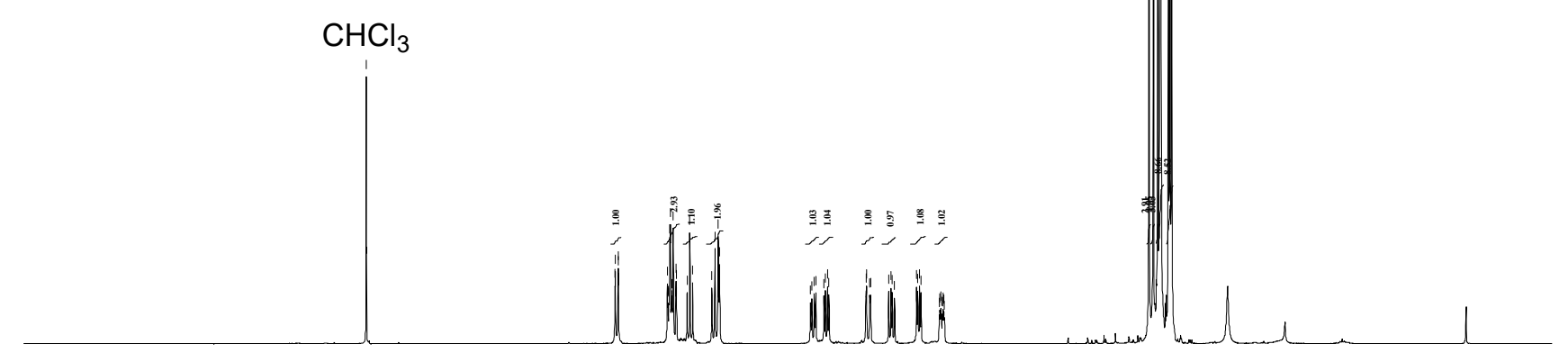

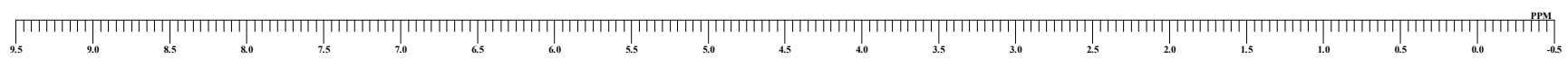

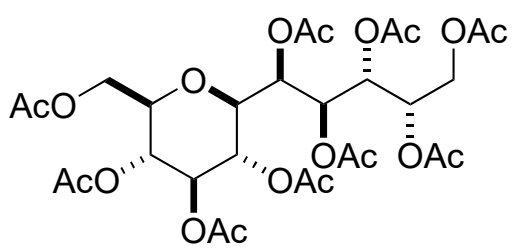

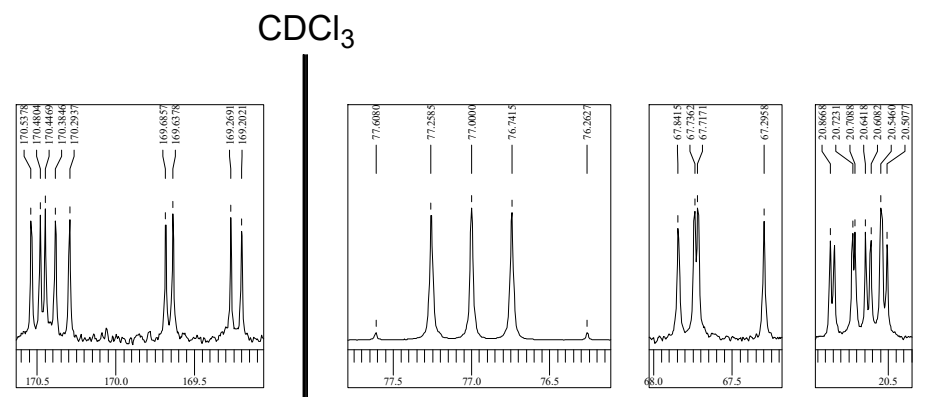

diospyrodin nonaacetate (2)

${ }^{13} \mathrm{C}$ NMR $\left(125 \mathrm{MHz}, \mathrm{CDCl}_{3}\right)$

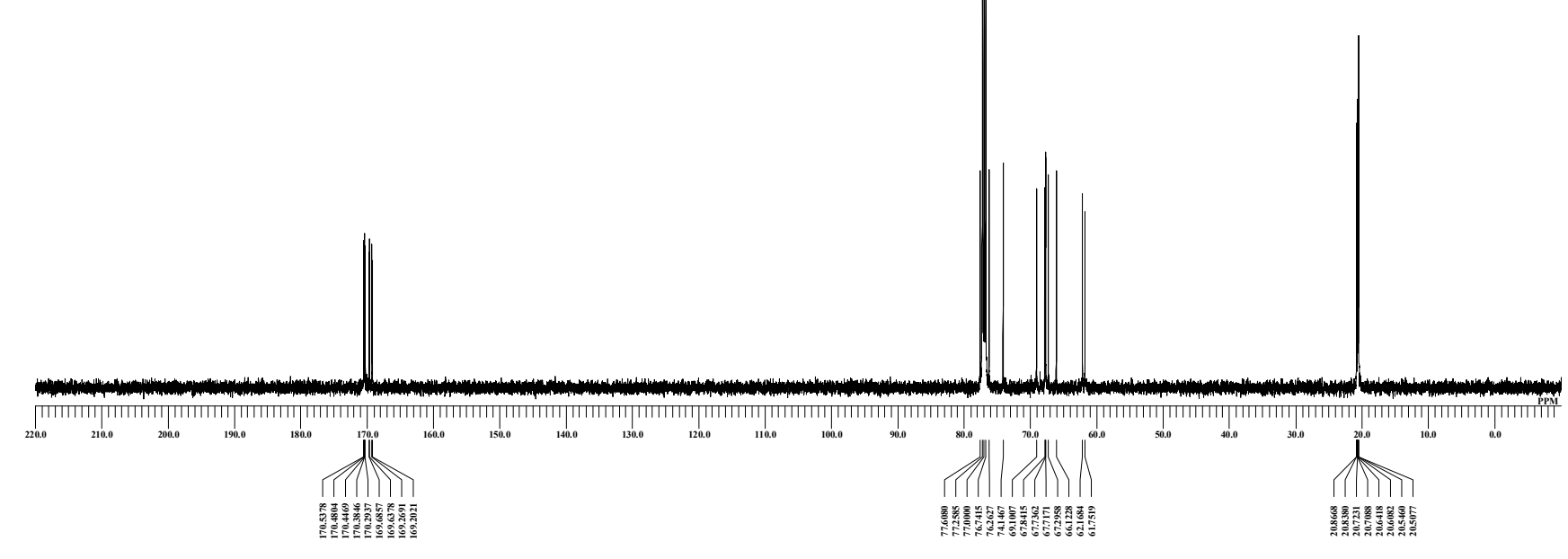




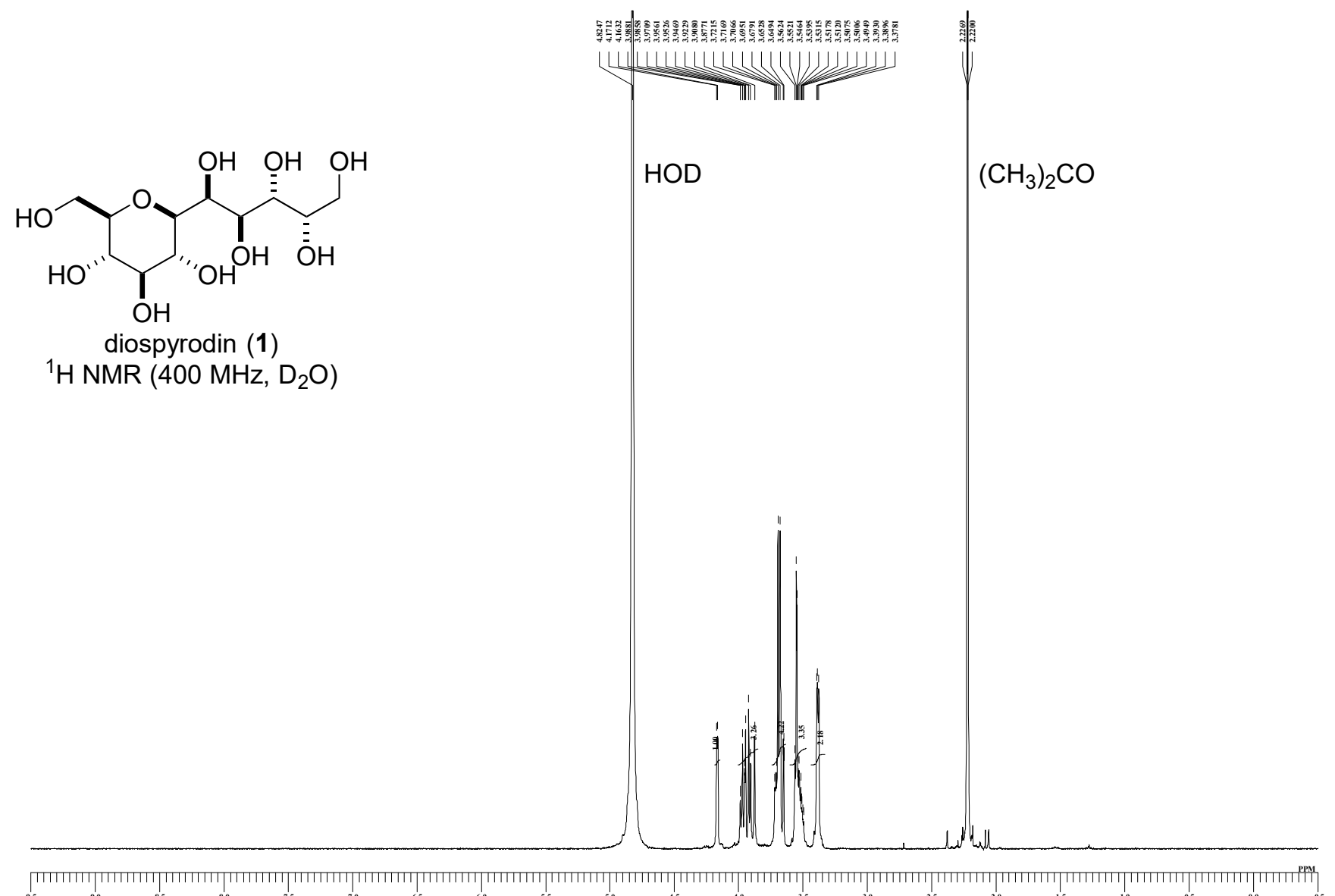

$\left(\mathrm{CH}_{3}\right)_{2} \mathrm{CO}$<smiles>OC[C@H](O)[C@@H](O)C(O)C(O)C1O[C@H](CO)[C@@H](O)C(O)[C@H]1O</smiles>

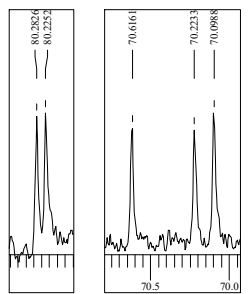

diospyrodin (1)

${ }^{13} \mathrm{C}$ NMR $\left(100 \mathrm{MHz}, \mathrm{D}_{2} \mathrm{O}\right)$

$\left(\mathrm{CH}_{3}\right)_{2} \mathrm{CO}$

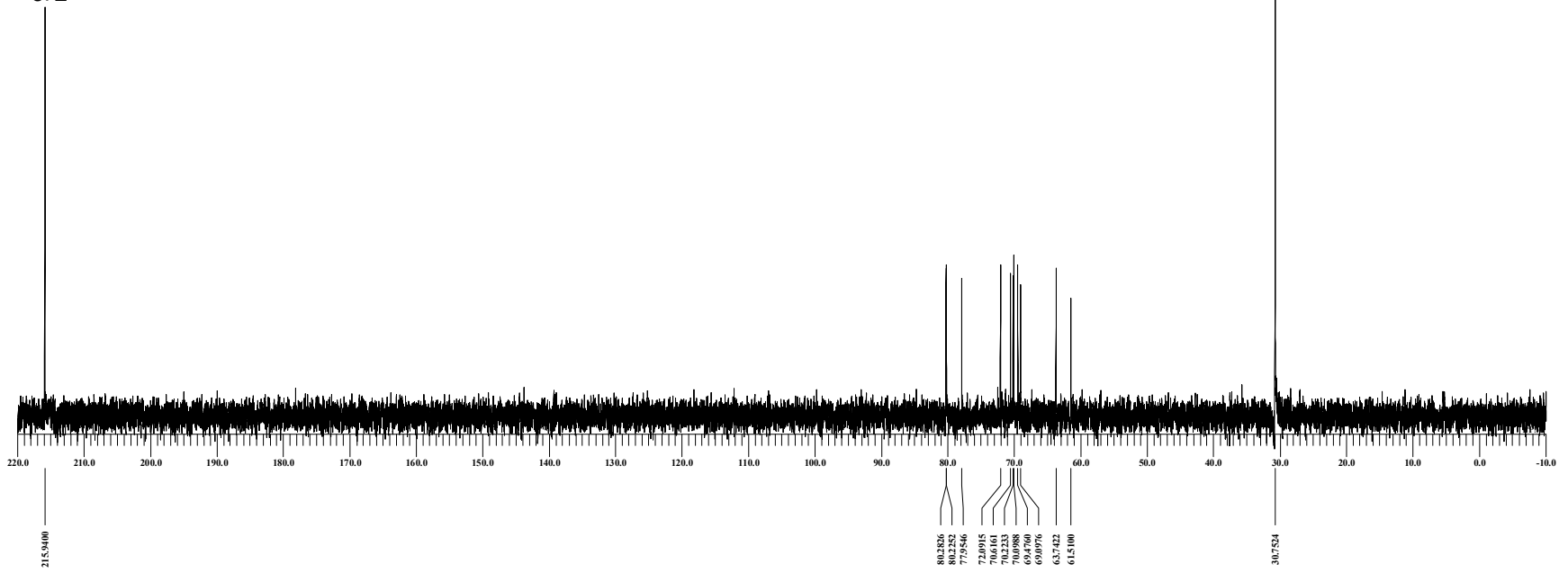




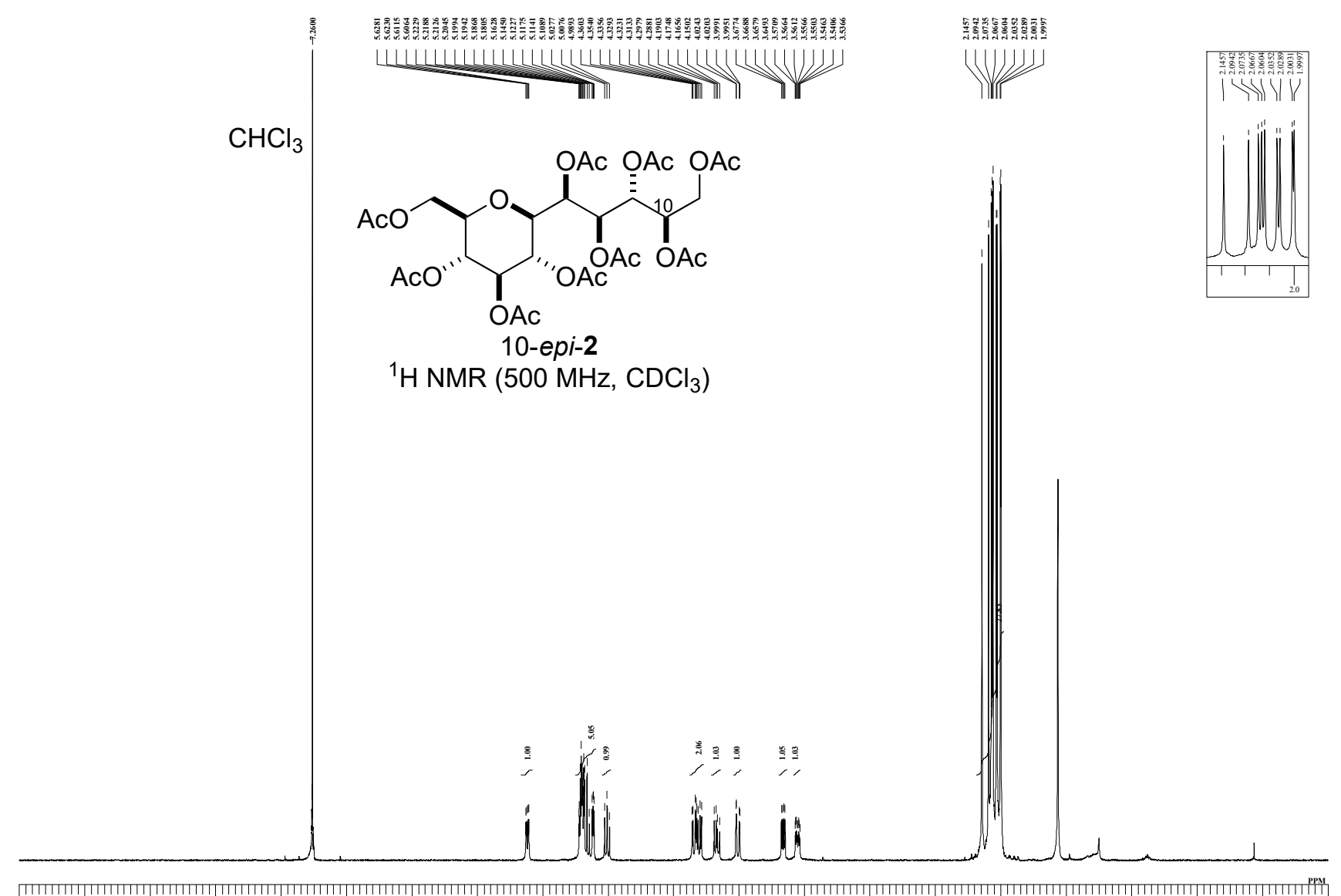

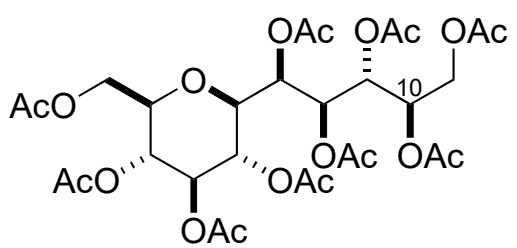

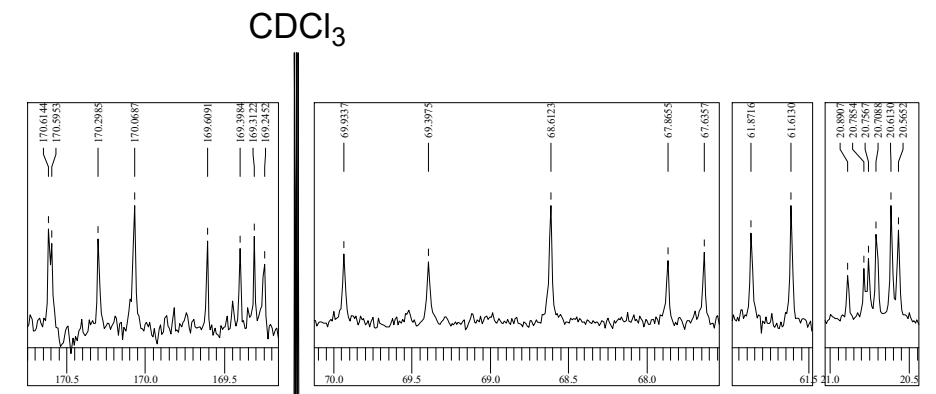

10-ері-2

${ }^{13} \mathrm{C}$ NMR (125 MHz, $\mathrm{CDCl}_{3}$ )

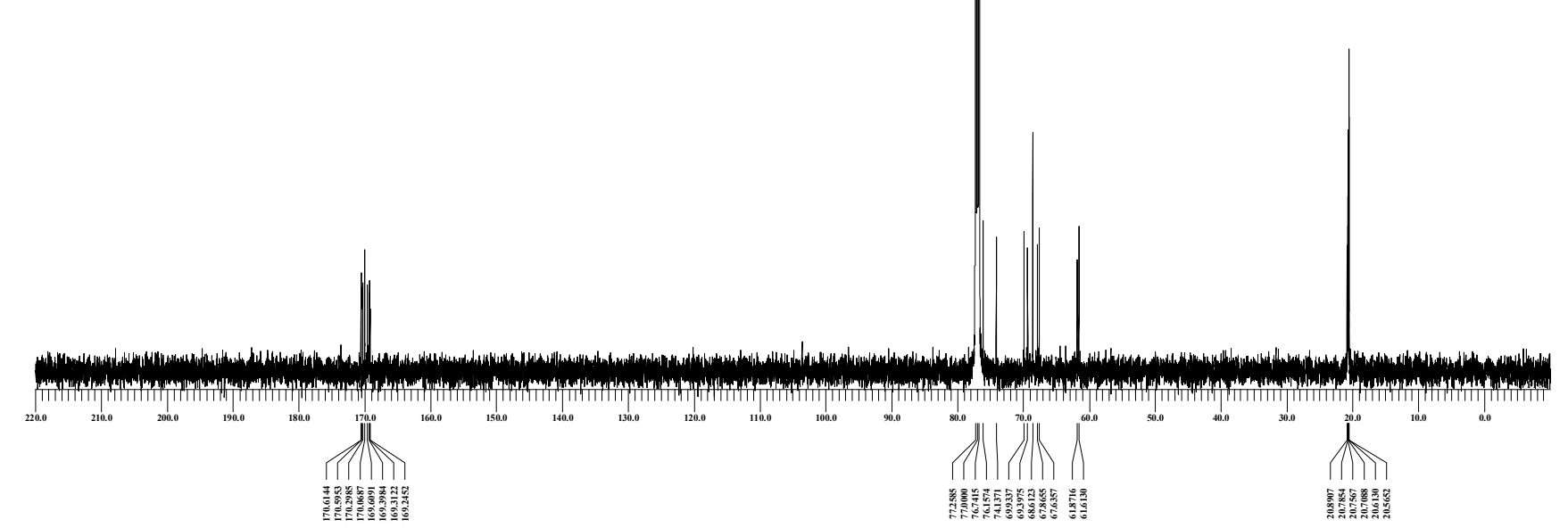



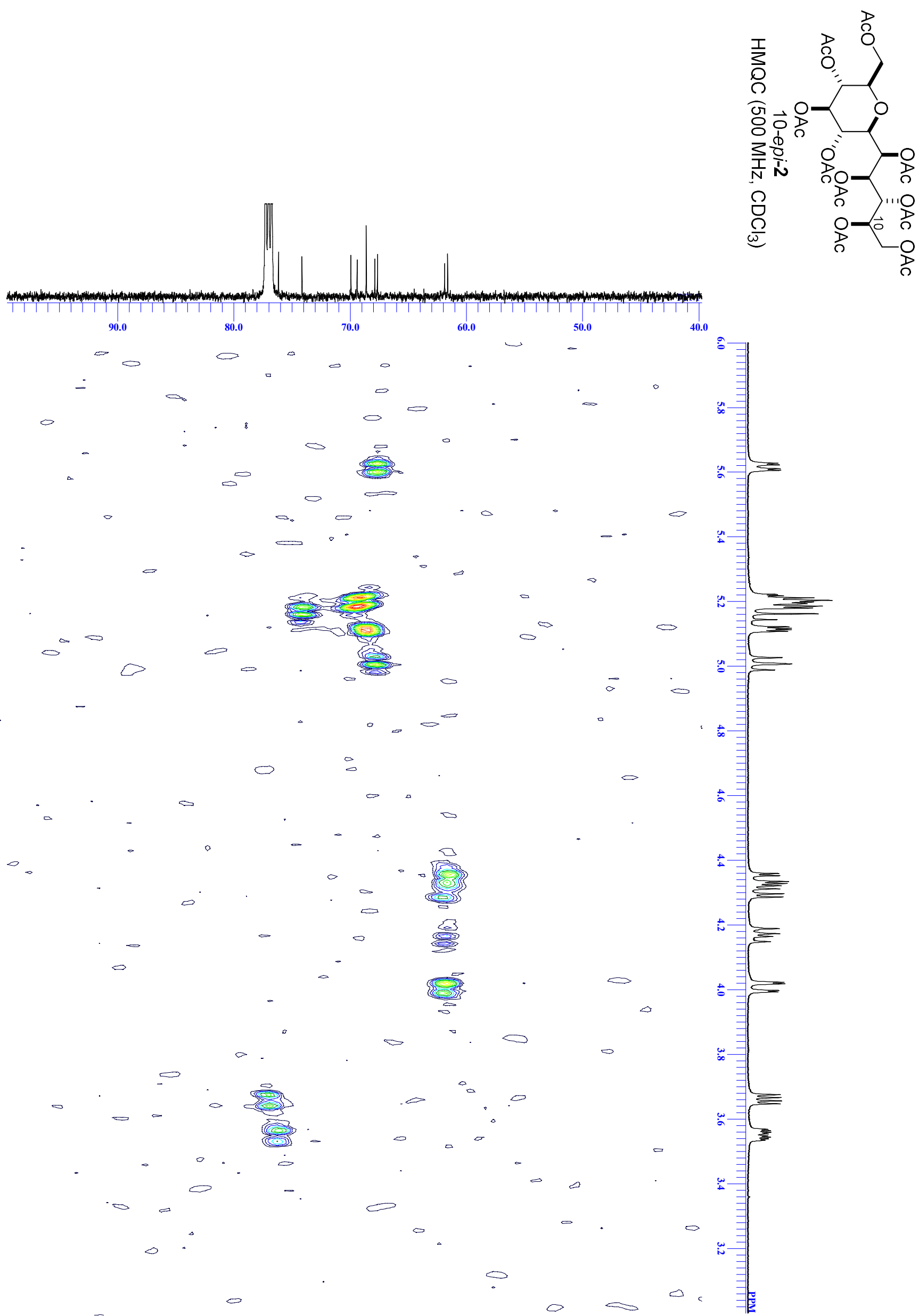


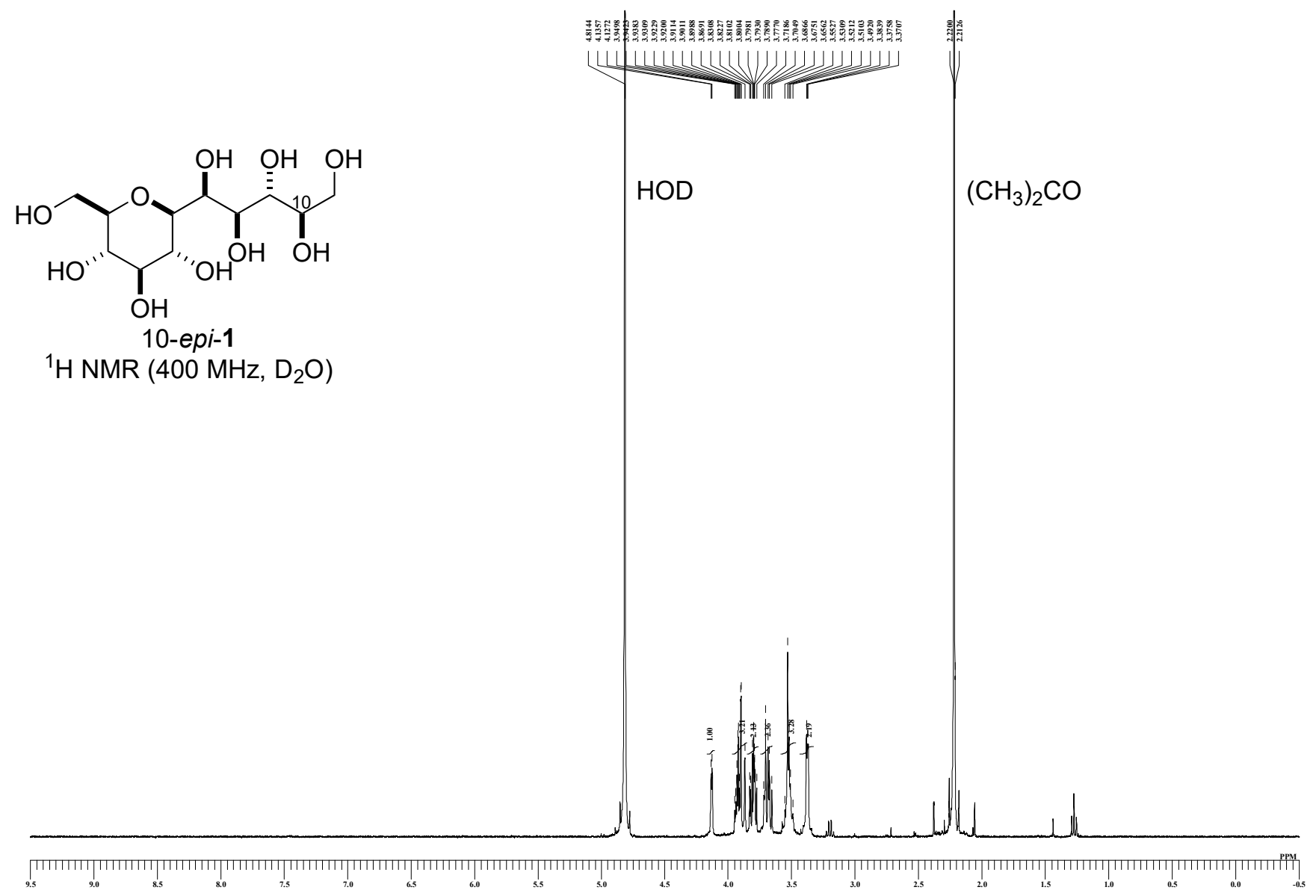

$\left(\mathrm{CH}_{3}\right)_{2} \mathrm{CO}$<smiles>OC[C@H](O)[C@@H](O)[C@H](O)[C@H](O)[C@H]1O[C@H](CO)[C@@H](O)C(O)[C@H]1O</smiles>

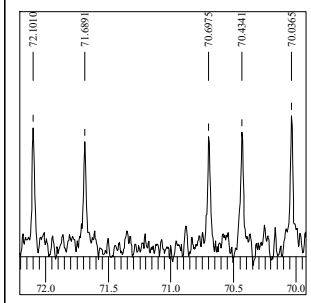

${ }^{13} \mathrm{C}$ NMR $\left(100 \mathrm{MHz}, \mathrm{D}_{2} \mathrm{O}\right)$

$\left(\mathrm{CH}_{3}\right)_{2} \mathrm{CO}$

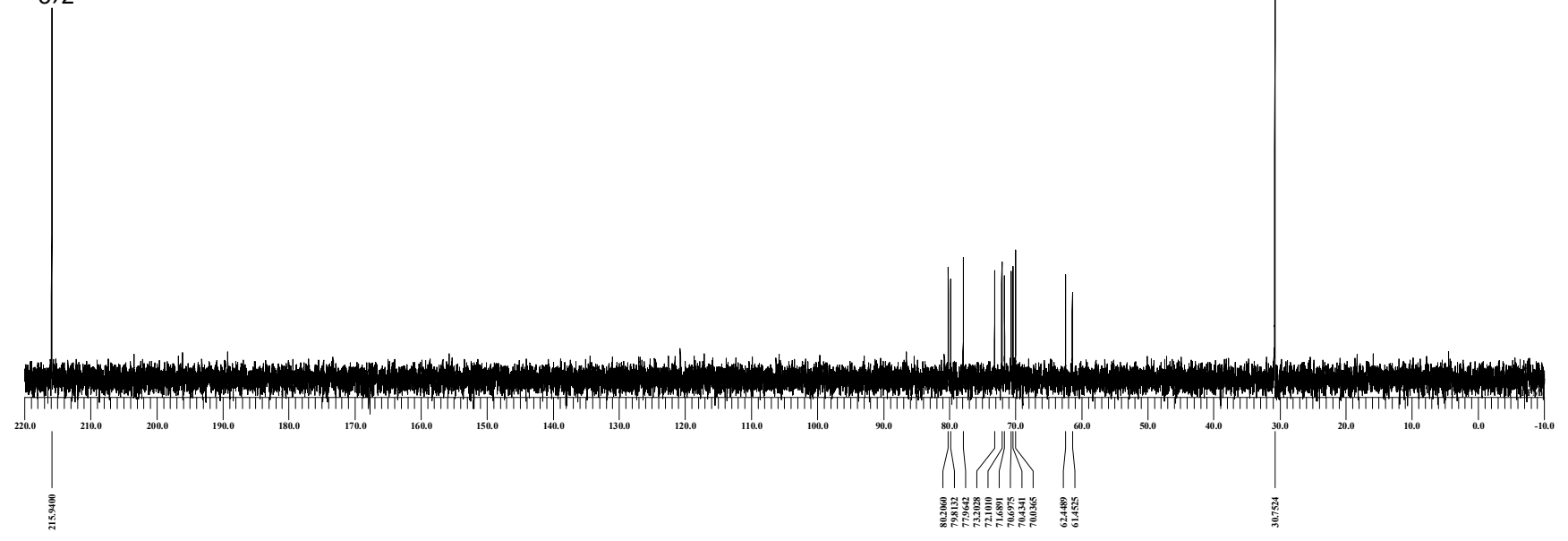




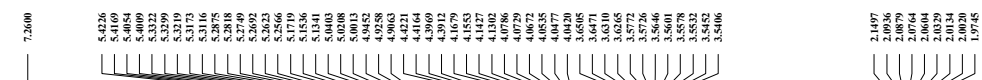

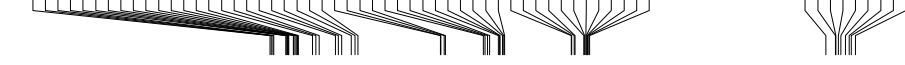

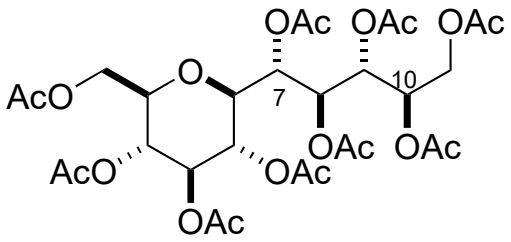

7,10-diepi-2

${ }^{1} \mathrm{H}$ NMR $\left(500 \mathrm{MHz}, \mathrm{CDCl}_{3}\right)$

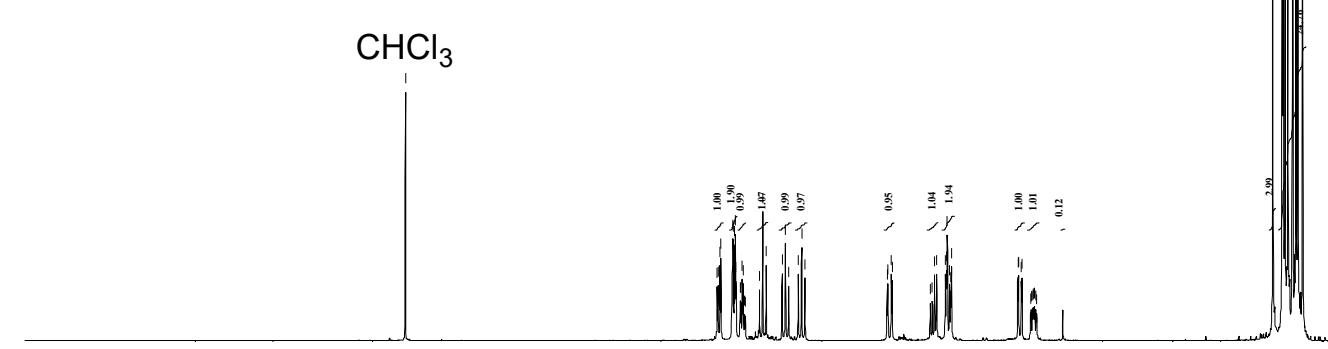

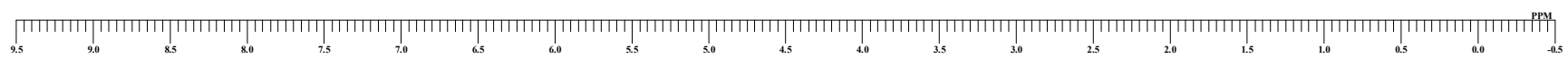

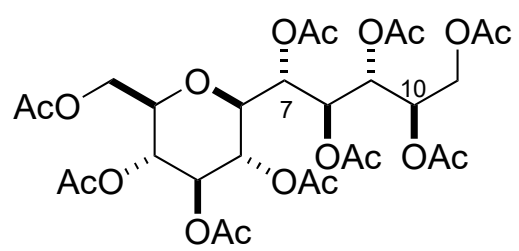

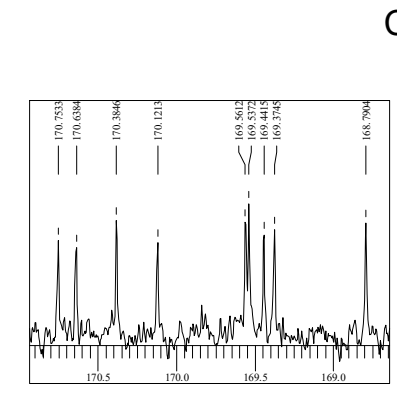

$\mathrm{CDCl}_{3}$

7,10-diepi-2

${ }^{13} \mathrm{C}$ NMR $\left(125 \mathrm{MHz}, \mathrm{CDCl}_{3}\right)$
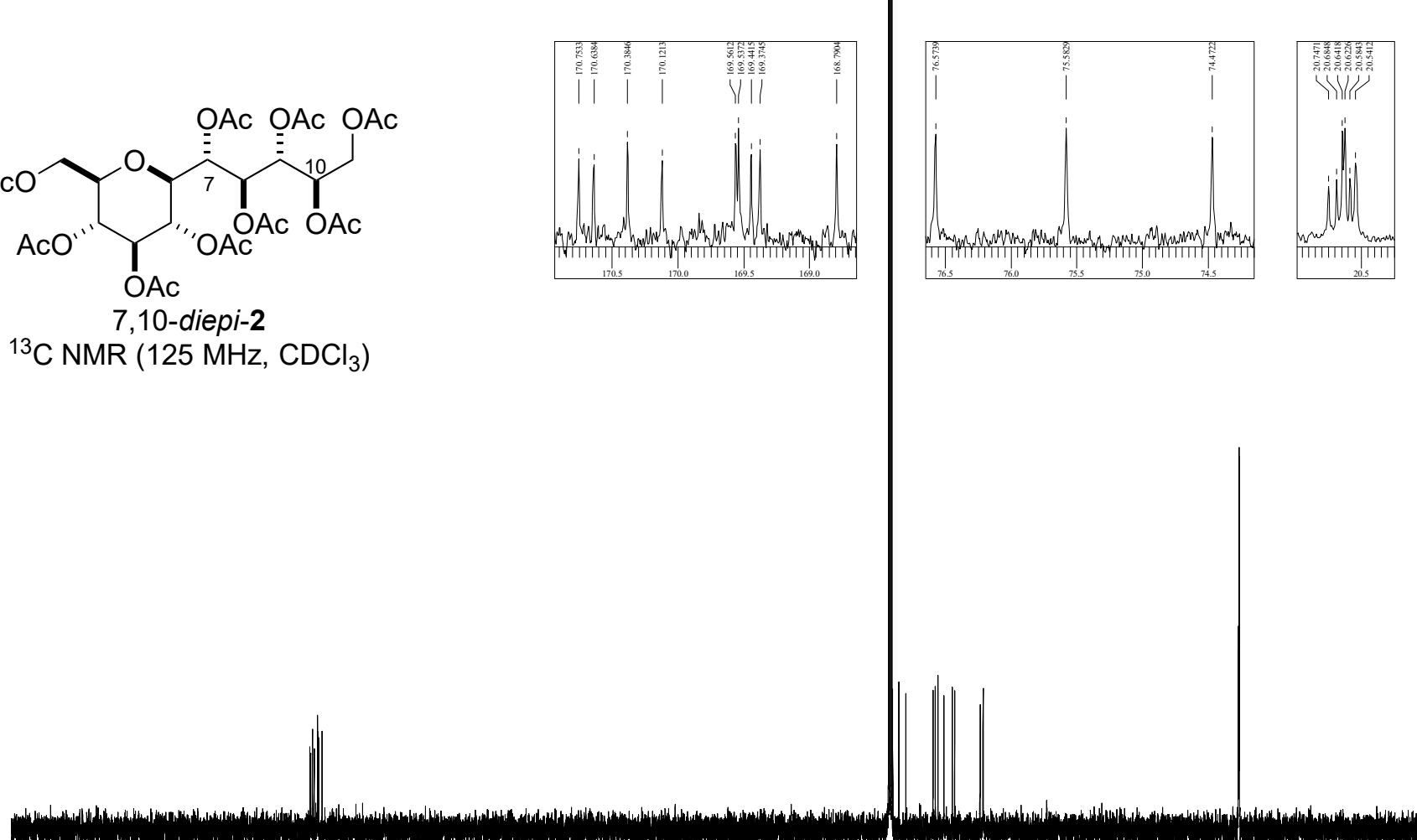
<smiles>OC[C@H](O)[C@@H](O)[C@H](O)[C@H](O)[C@H]1O[C@H](CO)[C@@H](O)C(O)[C@H]1O</smiles>

7,10-diepi-1

${ }^{1} \mathrm{H}$ NMR $\left(400 \mathrm{MHz}, \mathrm{D}_{2} \mathrm{O}\right)$

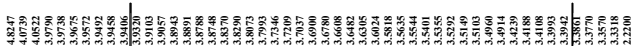

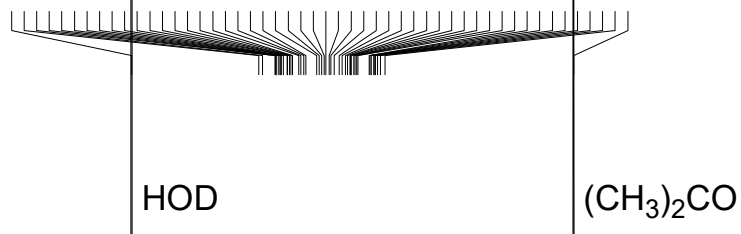

$\left(\mathrm{CH}_{3}\right)_{2} \mathrm{CO}$<smiles>OC[C@H](O)[C@@H](O)[C@H](O)[C@H](O)[C@H]1O[C@H](CO)[C@@H](O)C(O)[C@H]1O</smiles>

7,10-diepi-1

${ }^{13} \mathrm{C}$ NMR $\left(100 \mathrm{MHz}, \mathrm{D}_{2} \mathrm{O}\right)$

$\left(\mathrm{CH}_{3}\right)_{2} \mathrm{CO}$

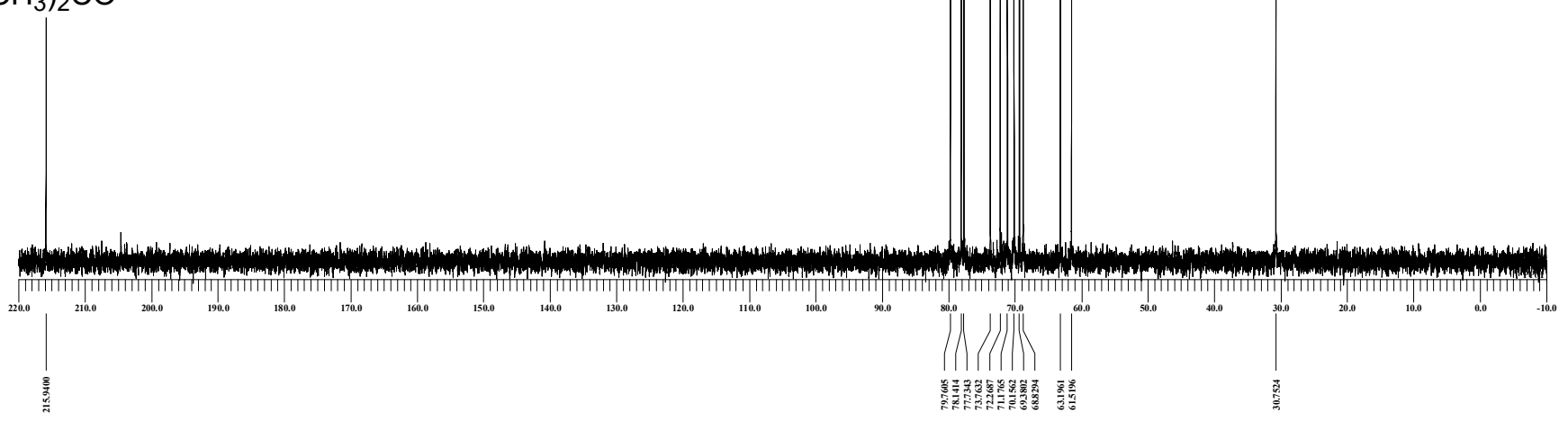




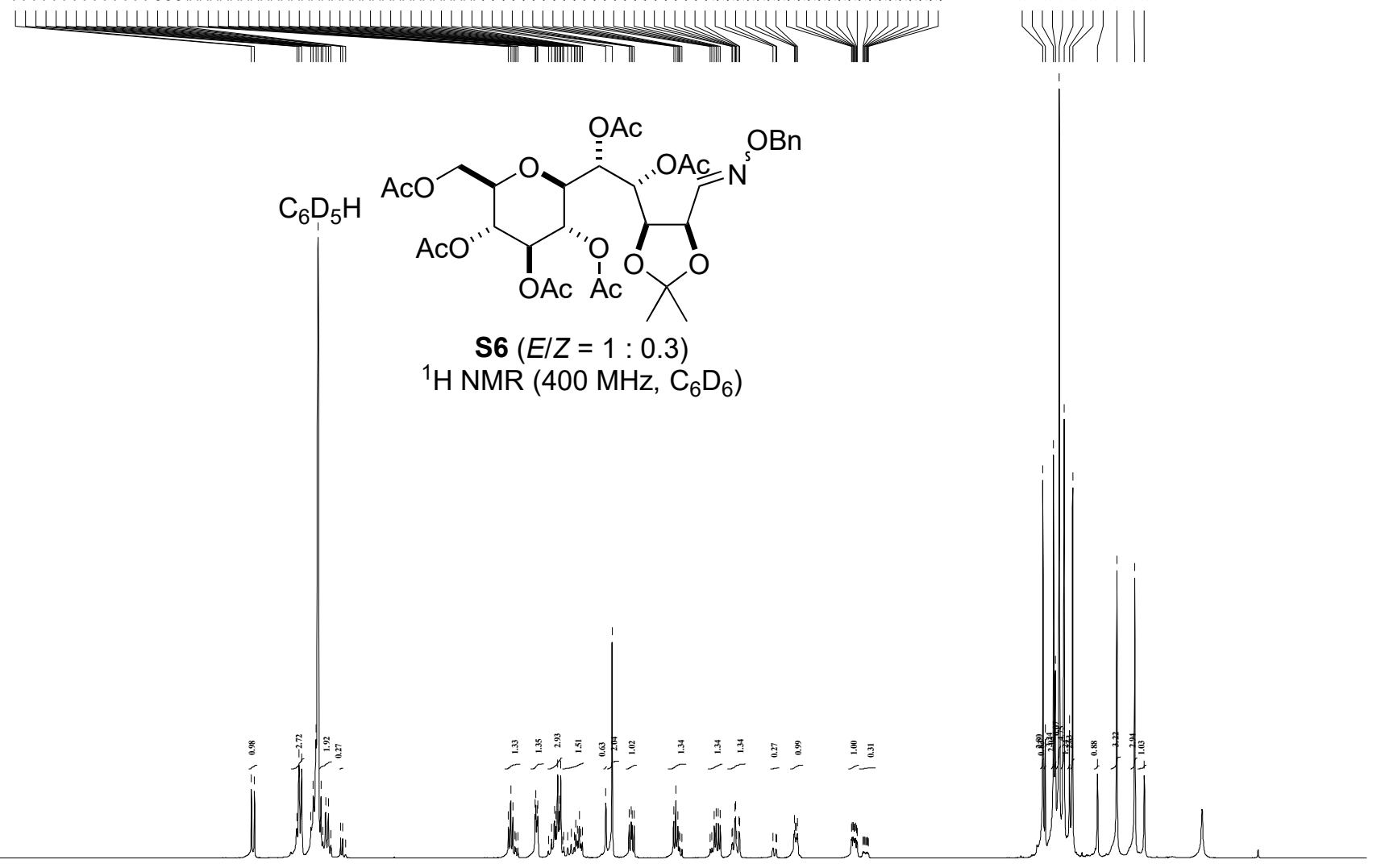

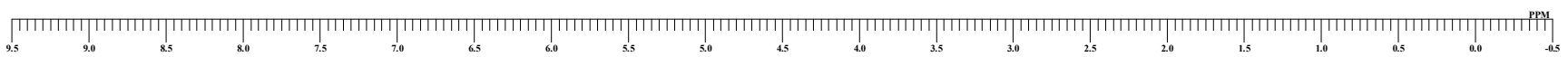

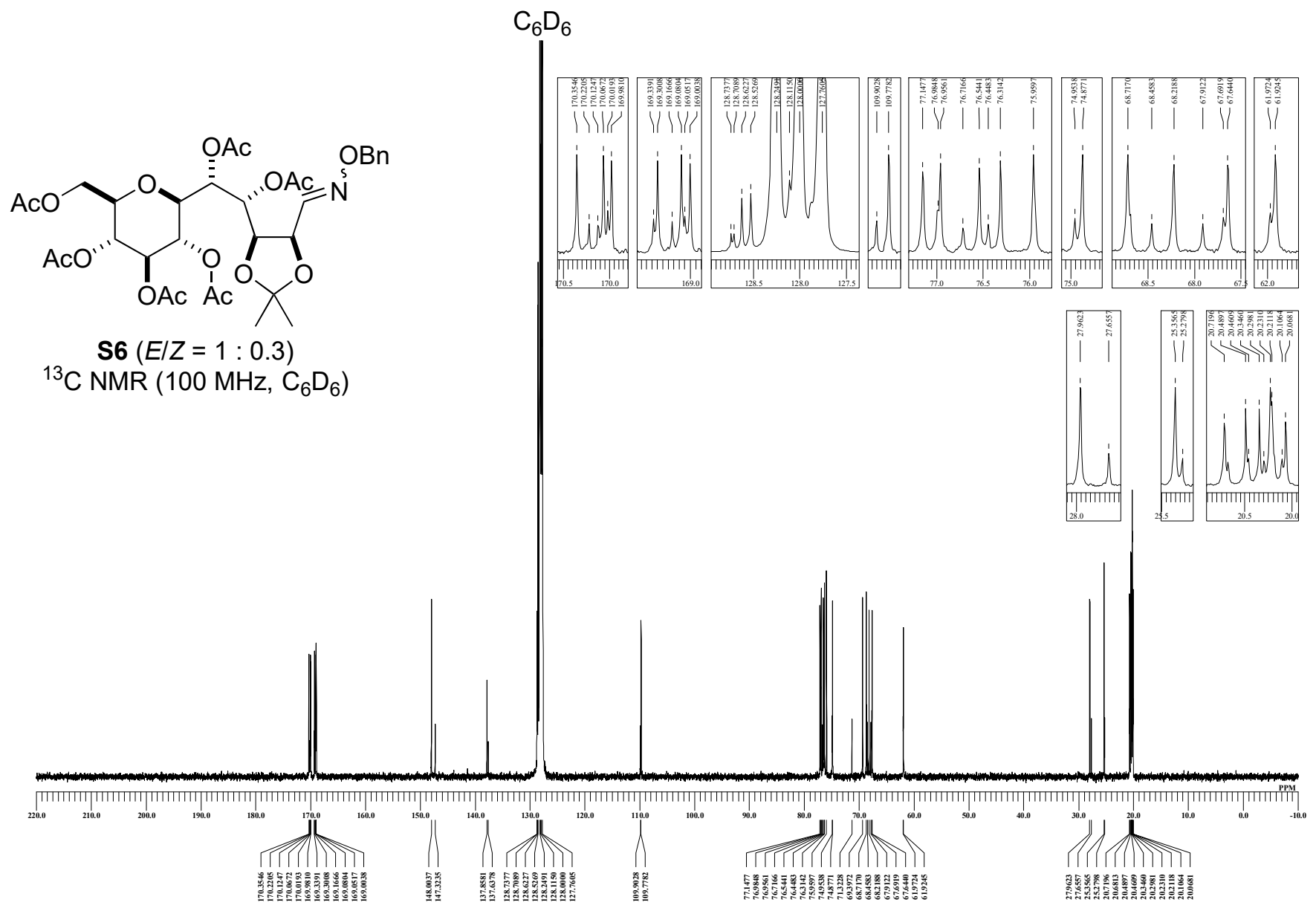



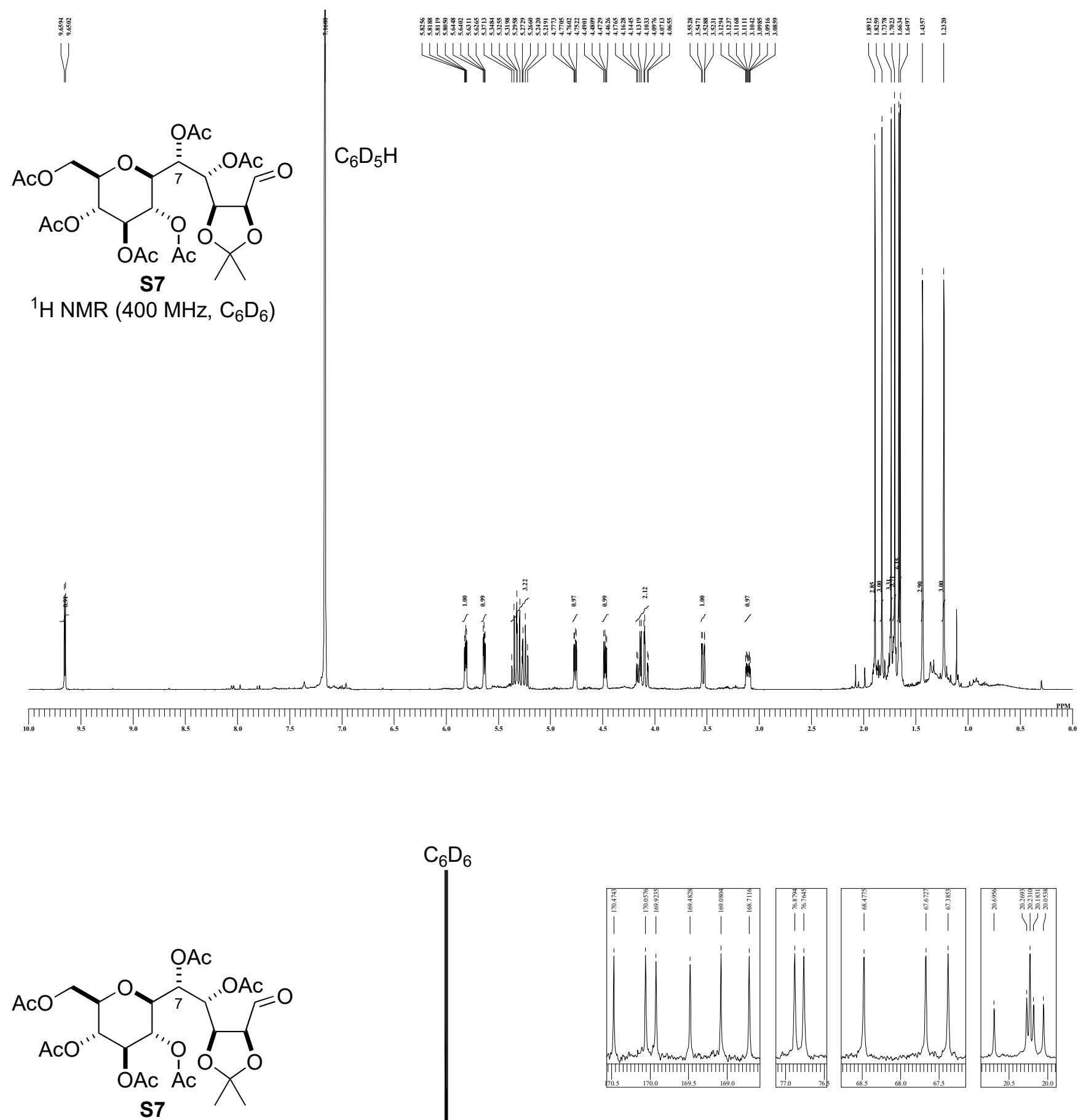

${ }^{13} \mathrm{C}$ NMR $\left(100 \mathrm{MHz}, \mathrm{C}_{6} \mathrm{D}_{6}\right)$

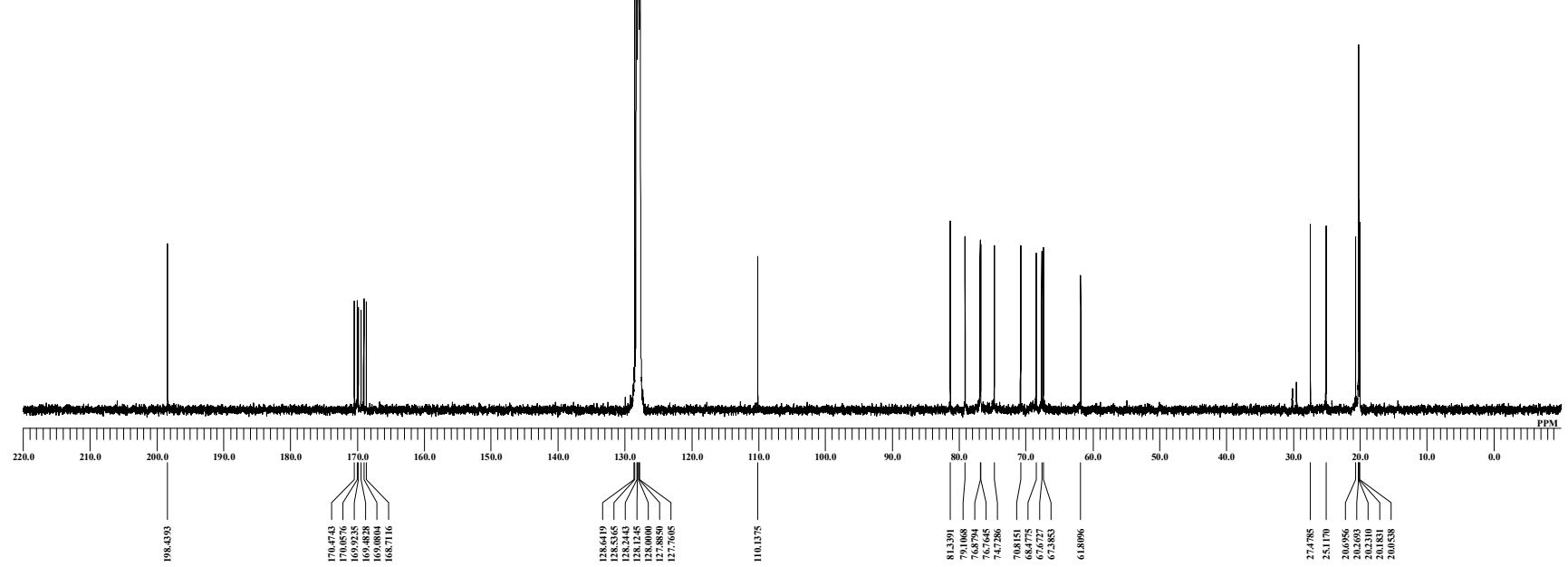



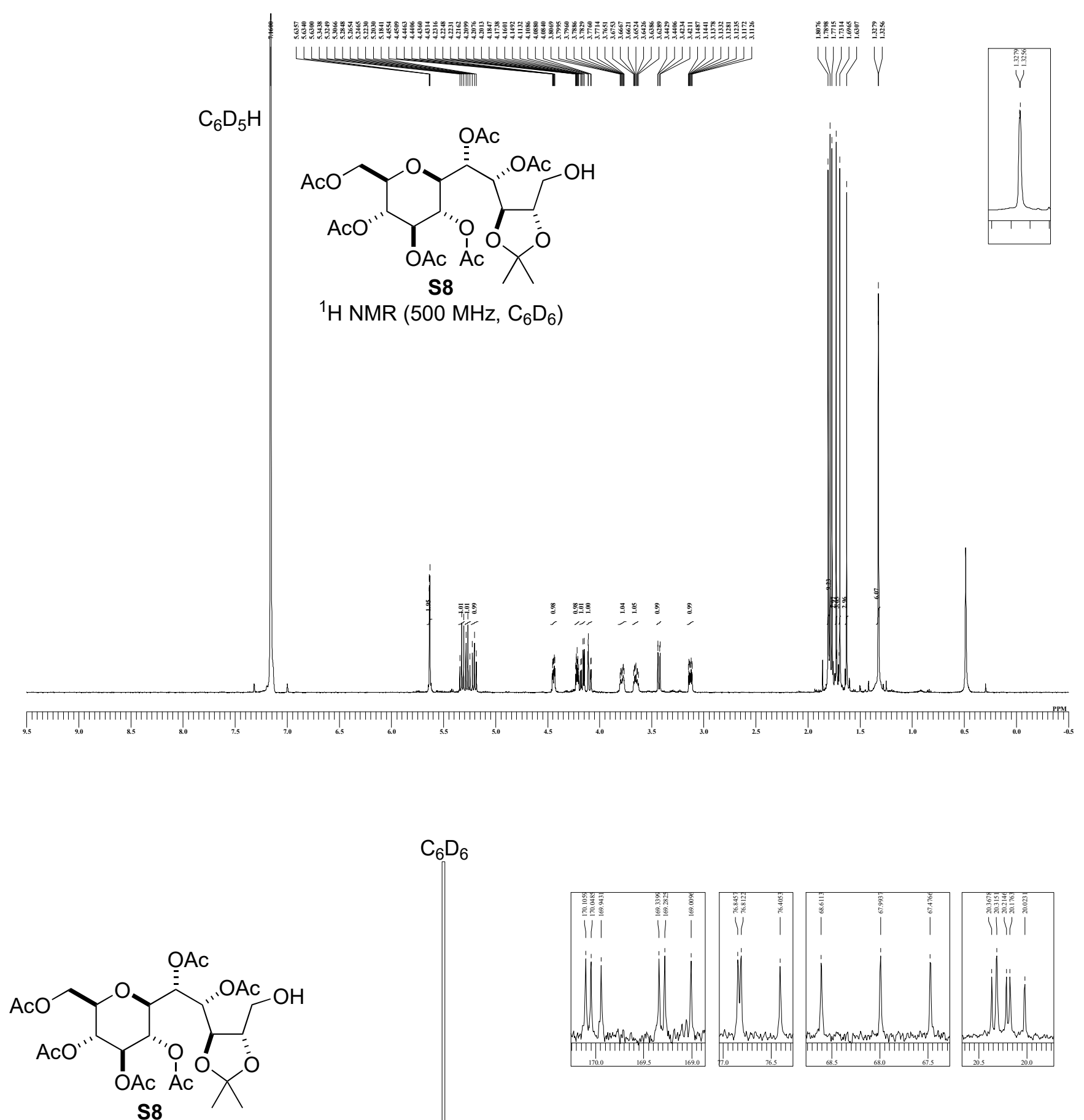

$\mathrm{C}_{6} \mathrm{D}_{6}$

${ }^{13} \mathrm{C}$ NMR $\left(125 \mathrm{MHz}, \mathrm{C}_{6} \mathrm{D}_{6}\right)$
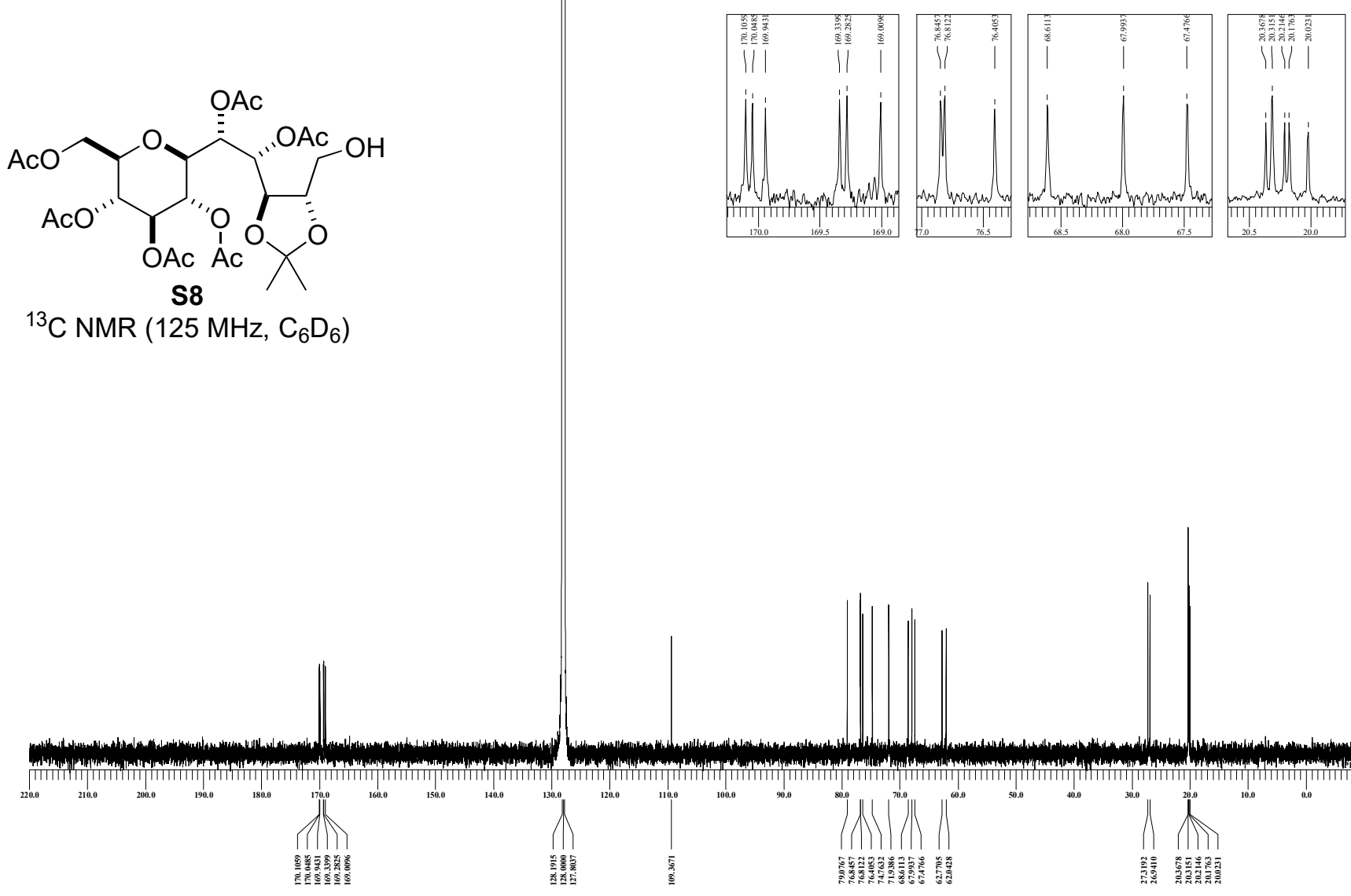


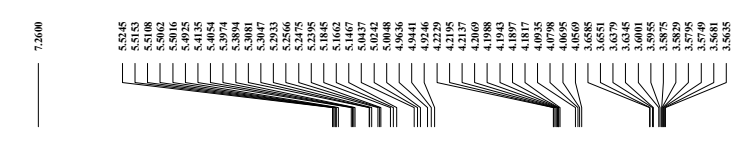<smiles>CC(=O)OCC1OC([C@H](OC(C)=O)C(OC(C)=O)C(OC(C)=O)C(C)OC(C)=O)[C@H](OC(C)=O)[C@@H](OC(C)=O)[C@@H]1OC(C)=O</smiles>

7-epi-2

${ }^{1} \mathrm{H}$ NMR $\left(500 \mathrm{MHz}, \mathrm{CDCl}_{3}\right)$

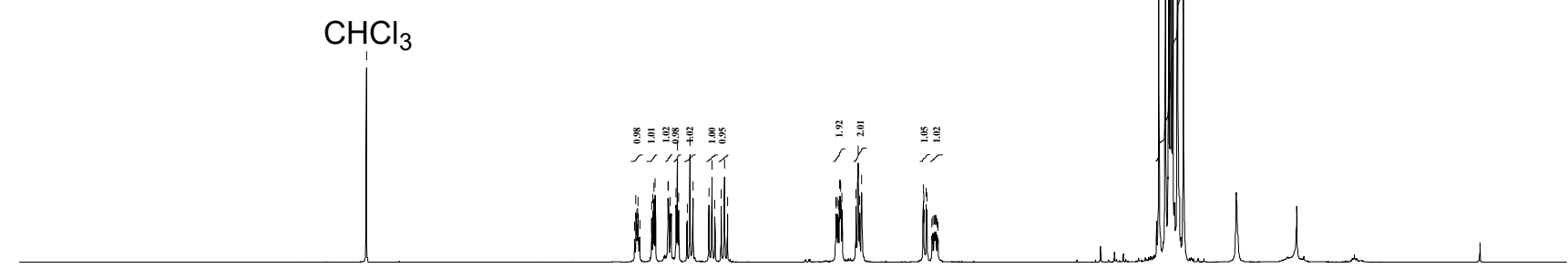

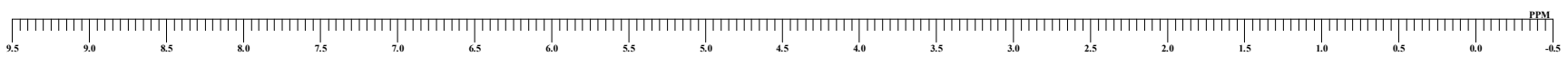

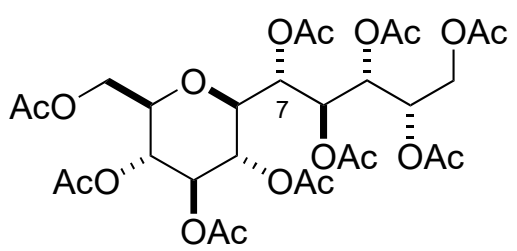

7-epi-2

${ }^{13} \mathrm{C}$ NMR $\left(125 \mathrm{MHz}, \mathrm{CDCl}_{3}\right)$
$\mathrm{CDCl}_{3}$
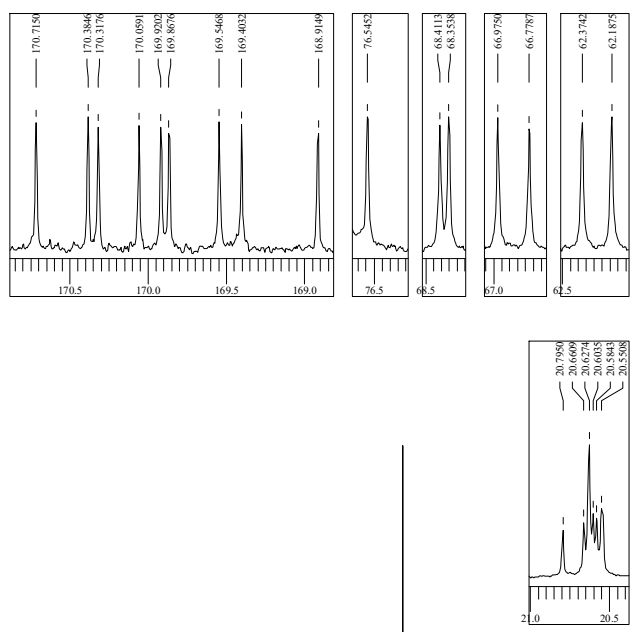$$
\prod_{220.0}
$$

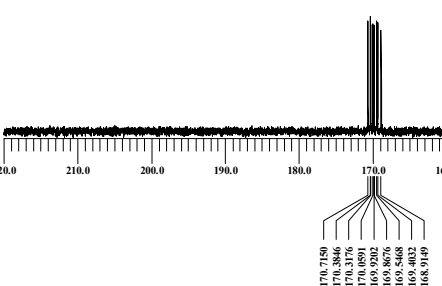

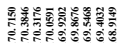

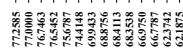




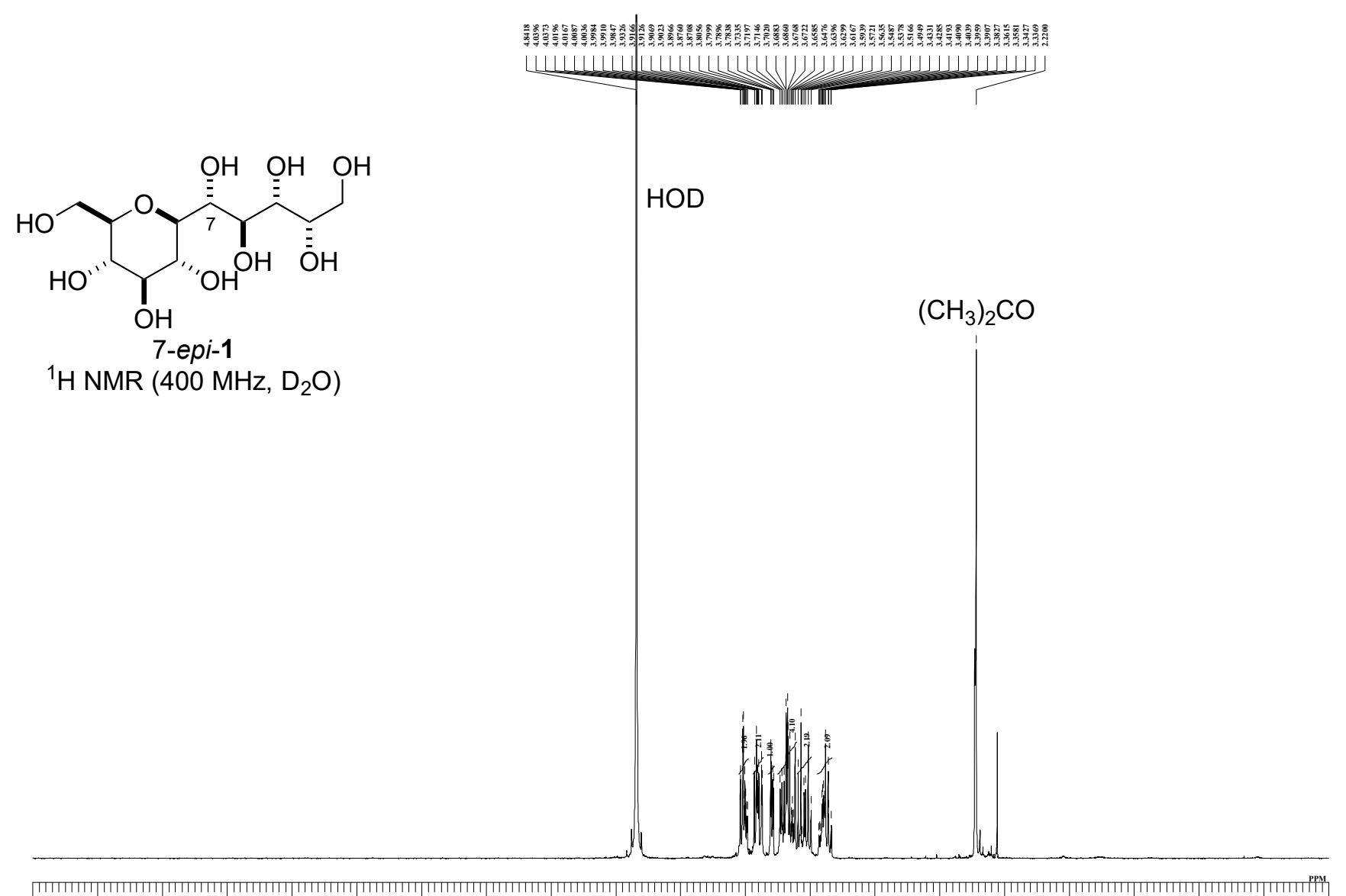<smiles>OCC(O)C(O)C(O)C(O)[C@H]1OC(CO)[C@@H](O)C(O)[C@H]1O</smiles>

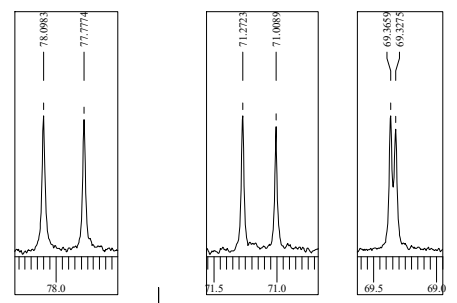

${ }^{13} \mathrm{C}$ NMR $\left(100 \mathrm{MHz}, \mathrm{D}_{2} \mathrm{O}\right)$

$\left(\mathrm{CH}_{3}\right)_{2} \mathrm{CO}$

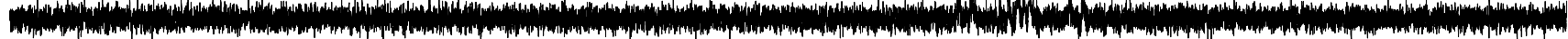

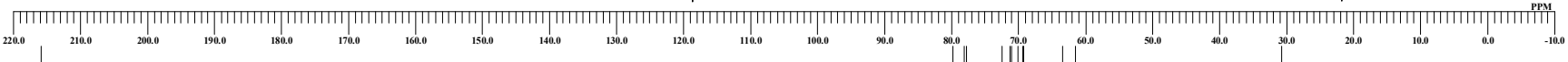



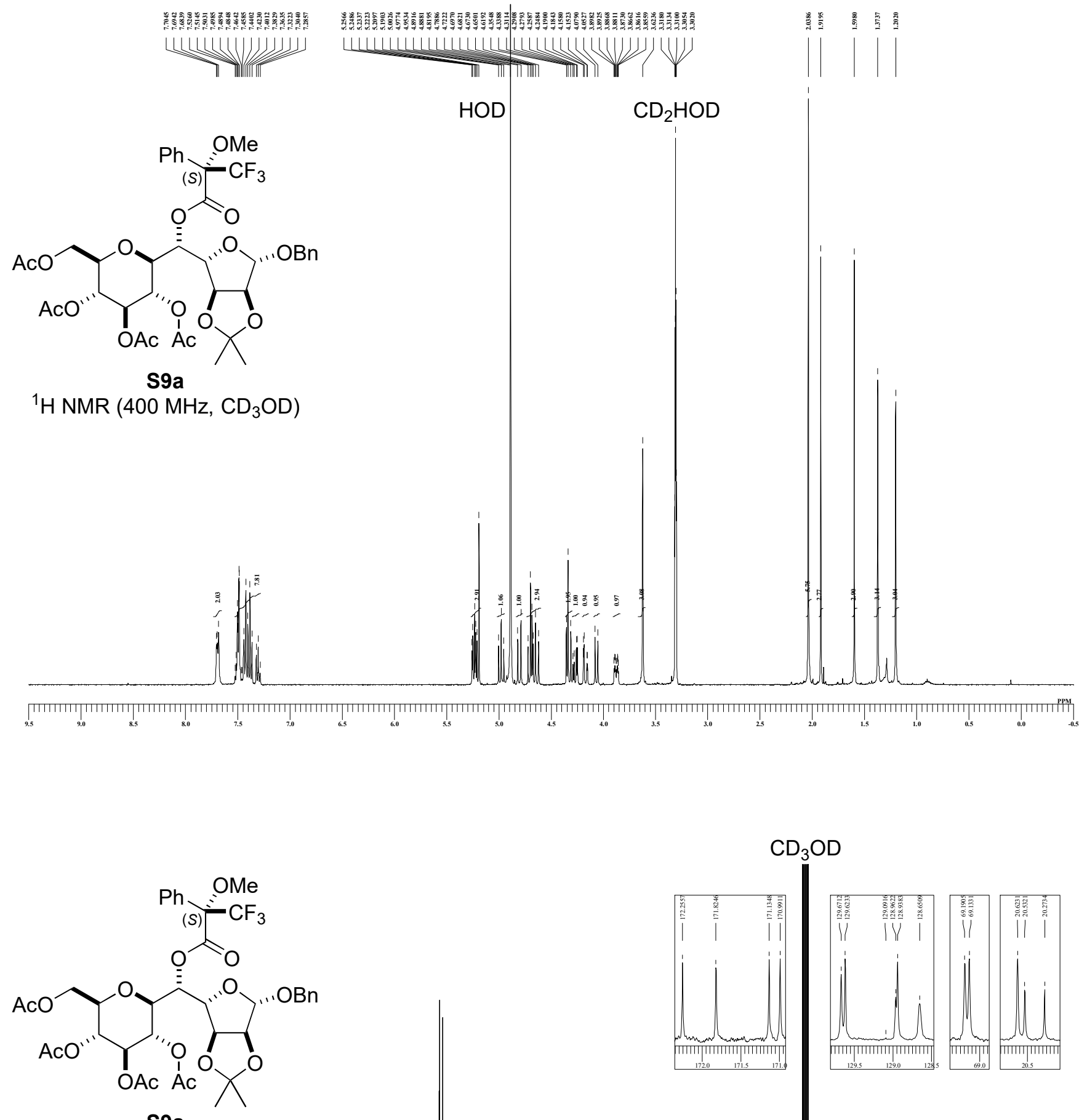

${ }^{13} \mathrm{C}$ NMR (100 MHz, $\left.\mathrm{CD}_{3} \mathrm{OD}\right)$

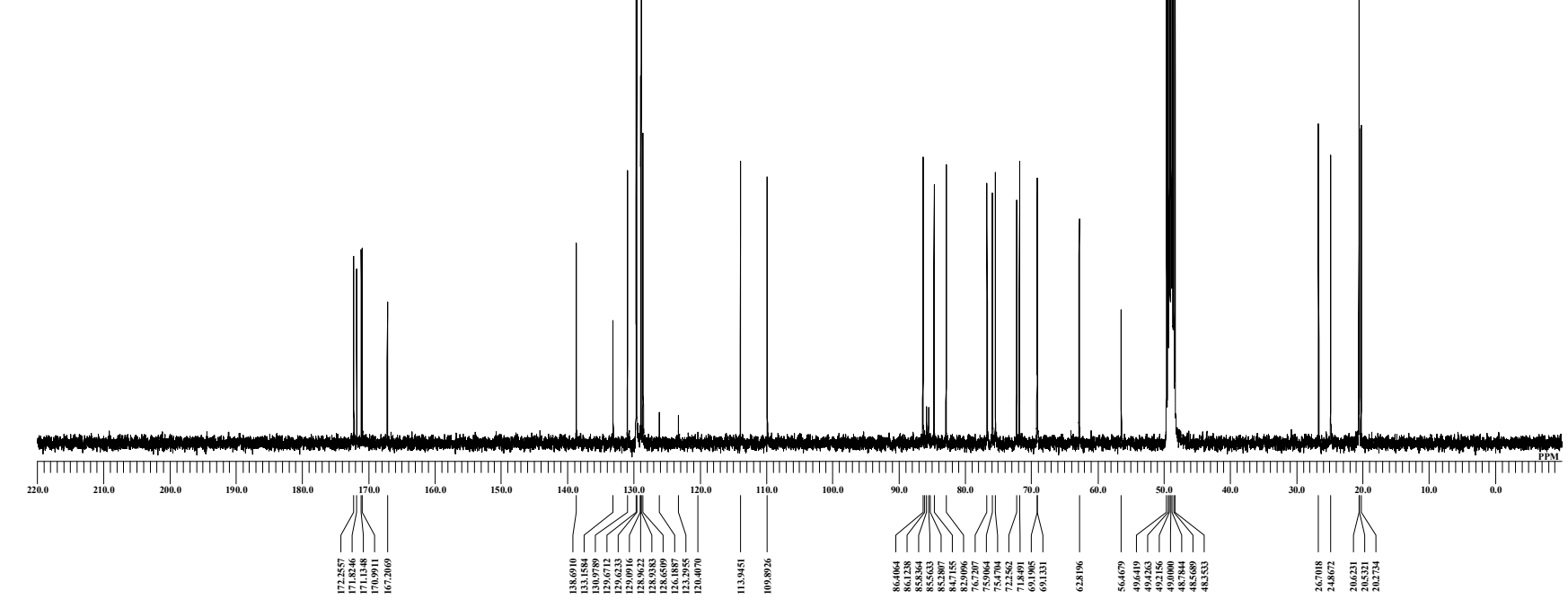



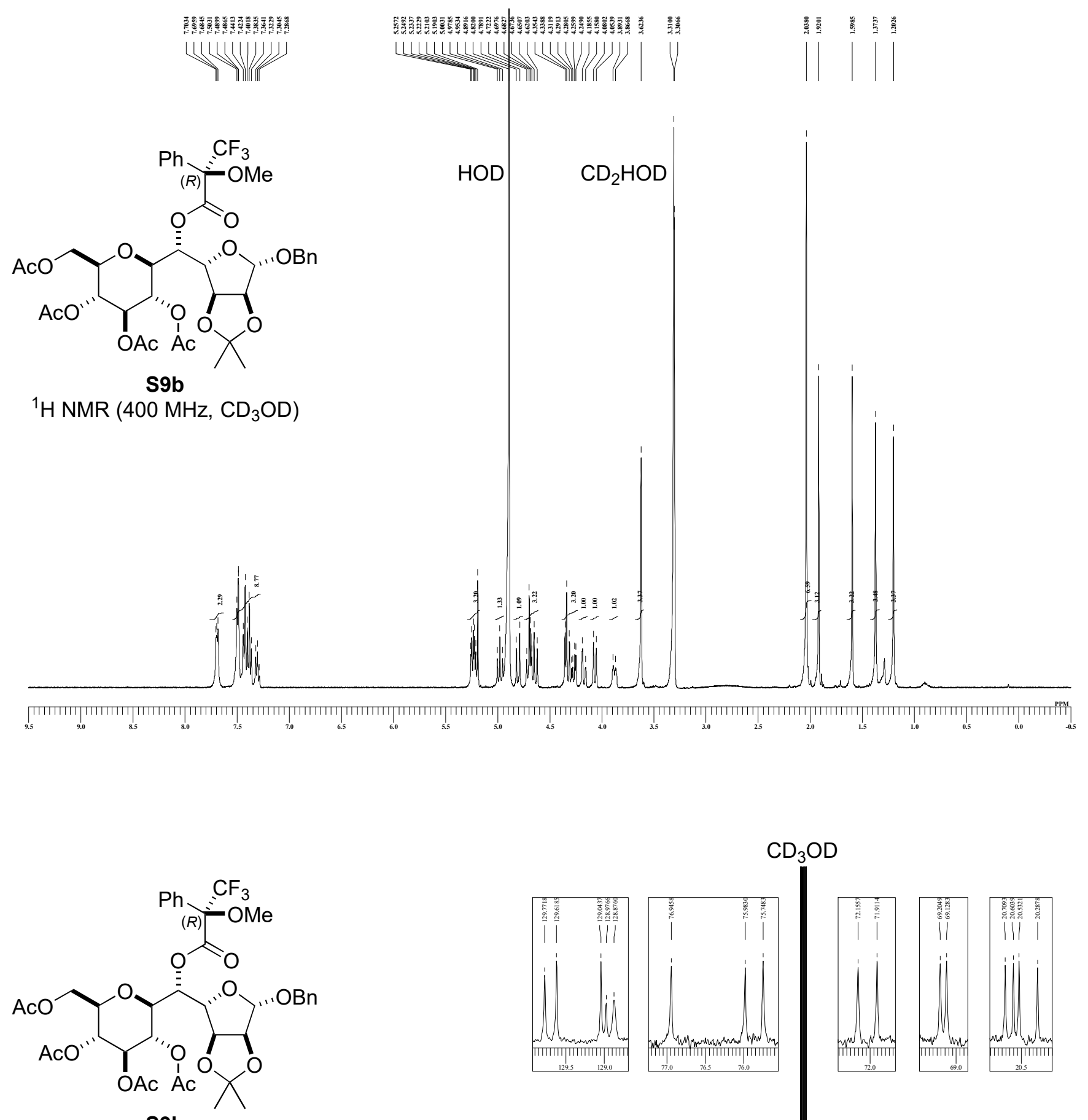

${ }^{13} \mathrm{C}$ NMR (100 MHz, CD $\left.{ }_{3} \mathrm{OD}\right)$

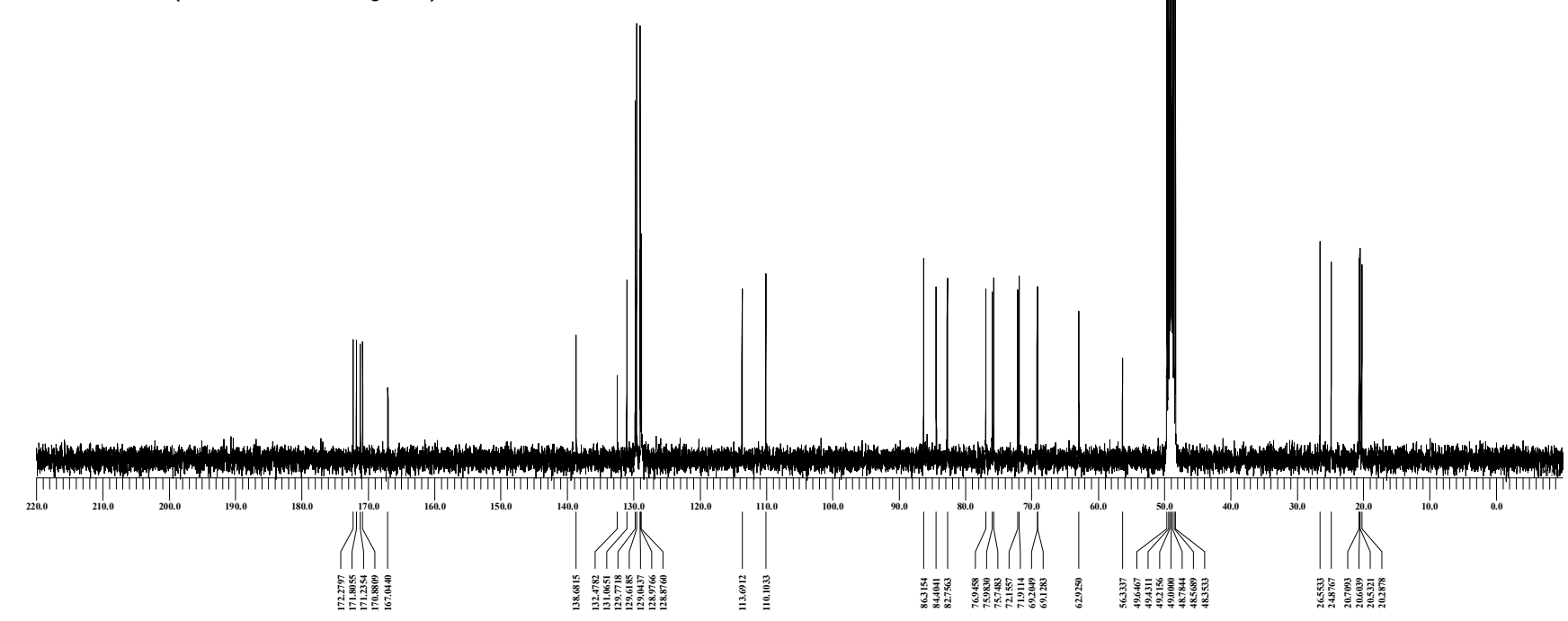

